\title{
Ion cyclotron resonance heating scenarios for DEMO
}

\section{JET Contributors}

2019-10

JET Contributors, Hatch, D R , Ahlgren , T , Aho-Mantila , L, Airila , M , Björkas , C , Heinola , K, Lahtinen, A, Nordlund, K, Safi , E , Pehkonen, S-P , Salmi , A, Tala , T , Likonen , J , Koivuranta , S , Hakola , A , Asunta , O , Chone , L , Groth , M , Karhunen , J , Kurki-Suonio , T , Leerink , S , Lomanowski, B , Lonnroth , J , Santala , M I K, Simpson , J , Sipila , S K , Varje , J \& Van Eester , D 2019 , ' lon cyclotron resonance heating scenarios for DEMO ' , Nuclear Fusion , vol. 59 , no. 10 , 106051 . https://doi.org/10.1088/1741-4326/ab318b

http://hdl.handle.net/10138/324306

https://doi.org/10.1088/1741-4326/ab318b

acceptedVersion

Downloaded from Helda, University of Helsinki institutional repository.

This is an electronic reprint of the original article.

This reprint may differ from the original in pagination and typographic detail.

Please cite the original version. 


\title{
Ion cyclotron resonance heating scenarios for DEMO
}

\author{
D. Van Eester ${ }^{1}$, E. Lerche ${ }^{1,2}$, R. Ragona ${ }^{1}$, \\ A. Messiaen ${ }^{1}, \mathrm{~T}$. Wauters ${ }^{1}$ and JET contributors* \\ EUROfusion Consortium, JET, Culham Science Centre, Abingdon, OX14 3DB, UK \\ ${ }^{1}$ LPP-ERM/KMS, EUROfusion Consortium Member, TEC, Brussels, Belgium \\ ${ }^{2} \mathrm{CCFE}$, Culham Science Centre, Abingdon, OX14 3DB, UK \\ *See the author list of "Overview of the JET preparation for Deuterium-Tritium Operation" \\ by E. Joffrin et al. to be published in Nuclear Fusion Special issue: overview and summary \\ reports from the 27th Fusion Energy Conference (Ahmedabad, India, 22-27 October 2018)
}

July 9,2019

\begin{abstract}
The present paper offers an overview of the potential of ion cyclotron resonance heating $(\mathrm{ICRH})$ or radio frequency $(\mathrm{RF})$ heating for the DEMO machine. It is found that various suitable heating schemes are available. Similar to ITER and in view of the limited bandwidth of about $10 \mathrm{MHz}$ that can be achieved to ensure optimal functioning of the launcher, it is proposed to make core second harmonic tritium heating the key ion heating scheme, assisted by fundamental cyclotron heating ${ }^{3} \mathrm{He}$ in the early phase of the discharge; for the present design of DEMO - with a static magnetic field strength of $B_{o}=5.855 \mathrm{~T}$ - that places the $T$ and ${ }^{3} \mathrm{He}$ layers in the core for $f=60 \mathrm{MHz}$ and suggests to center the bandwidth around that main operating frequency. In line with earlier studies for hot, dense plasmas in large-size magnetic confinement machines it is shown that good single pass absorption is achieved but that the size as well as operating density and temperature of the machine cause the electrons to absorb a non-negligible fraction of the power away from the core when core ion heating is aimed at. Current drive and alternative heating options are briefly discussed and a dedicated computation is done for the traveling wave antenna, proposed for DEMO in view of its compatibility with substantial antenna-plasma distances. The various tasks that ICRH can fulfill are briefly listed. Finally, the impact of transport and the sensitivity of the obtained results to changes in the machine parameters is commented on.
\end{abstract}

\section{Introduction}

DEMO will be the first power station based on harvesting energy from controlled fusion reactions. Whereas its predecessor ITER will have pulses lasting a few minutes 
- this already being much longer than what is typically achieved in most current-day machines - and will prove that the fusion power produced in a tokamak can be well beyond the power needed to start the fusion fire (an important next step towards commercialisation of fusion), DEMO should ideally operate for hours in a row and might - depending on the state-of-the-art of its subsystems available at the time it will actually be assembled - even be operated continuously rather than pulsed while being capable to feed several hundreds of MWs of power to the electricity grid, the projected fusion power it is designed to generate being of the multi-GW level (see e.g. [1] quoting $2 h$ pulses, $500 \mathrm{MW}$ of net electric output power and $50 \mathrm{MW}$ of auxiliary heating power installed). In view of the fairly agressive timeline EUROfusion proposes to arrive at the first actual power station relying on fusion [2], DEMO's preliminary design needs to be started well in advance. Other than for ITER, neutron fluences of the level expected in a working reactor need to be accounted for, requiring plasma facing components that withstand such loads. The design of a proper breeding blanket - ensuring tritium can be replenished inside the plant ("T self-sufficiency") - requires a sufficiently detailed and careful study of the integration of all components that can potentially reduce the tritium breeding ratio. As heating systems must be installed facing the plasma but be fed from behind the biological shield, their integration is carefully monitored. Depending on the source consulted, auxiliary heating power levels between 50 and $170 M W$ will be available to ensure DEMO's smooth operation in all its phases. DEMO will not yet be a commercial power station; its successor - typically referred to as PROTO - will.

In view of the need/desire to incorporate newly available knowledge whenever possible, the parameters of the DEMO machine tend to be changing as a function of time (see e.g. $[3,1]$ in which both a pulsed and a steady state configuration are considered). Various components of this machine will only be truly frozen when return of experience from actual experiments on the ITER machine will be available. In the present study - focussing on the role ion cyclotron resonance heating can play - the DEMO parameters as provided in the Physics and Magnetics Baseline document made available in February 2018 [4] and based on PROCESS [5] modelling will be considered. This machine is pulsed and labeled as "DEMO-1" (as opposed to a steady-state "DEMO-2" version) and has a major (geometric) radius of $R_{o}=9.002 \mathrm{~m}$ and a minor radius of $a_{p}=2.904 m$, yielding an aspect ratio of 3.1. The elongation is $\kappa=1.65$ and the triangularity $\delta=0.3333$. The toroidal magnetic field strength is $B_{o}=5.855 T$ at the geometric axis and the reference plasma current is $17.75 \mathrm{MA}$ corresponding to a near-edge safety factor $q_{95}$ of 3.887. In the activated phase and using balanced $D$ and $T$ concentrations this yields core temperatures of $T_{o}=28 \mathrm{keV}$ with a temperature profile factor of $\alpha_{T}=1.3$ for a central electron density of $N_{0, e}=10^{20} / \mathrm{m}^{3}$, pedestal density of $N_{s, e}=5.696 \times 10^{19} / \mathrm{m}^{3}$ and a density profile factor $\alpha_{N}=0.3$. Fusion reactions add fast alpha particles to the mix. Via Coulomb collisional slow down - mainly on the electrons in view of their high birth energy of $3.45 \mathrm{MeV}$ - these give rise to a non-negligible plasma dilution by ${ }^{4} \mathrm{He}$ ash. The concentration of the latter is of the order of $6 \%$ and the concentration of the fast subpopulation will be assumed to be $1 \%$, the energy confinement time being several seconds. Although the above PROCESS reference DEMO plasma was assumed to be heated by $50 \mathrm{MW}$ of electron cyclotron heating, neutral beam injection is expected to contribute to the plasma heating. A concentration of $2 \%$ of fast beam $D$ ions is considered. Both the beams and the fusion 
born alpha particles are modeled as Maxwellians, respectively considering them to be approximated sufficiently adequately by 200 and $600 \mathrm{keV}$ Maxwellian populations to qualitatively account for birth velocity as well as their Coulomb collisional slowing down; this choice is justified in a separate section. The reference computation equally accounts for minute amounts of Xenon $\left(X[X e]=N_{X e} / N_{e}=3.5 \times 10^{-4}\right)$ and Tungsten $\left(X[W]=N_{W} / N_{e}=5 \times 10^{-5}\right)$. Such small amounts of high $Z$ (charge) ions are believed not to play a role in the direct RF heating physics but significantly influence the performance of the scenario as they give rise to radiation: having too large concentrations of high $Z$ impurities in the plasma yields excessive radiation and potentially causes radiative collapse of the discharge. Medium Z impurities such as Argon may purposely be injected in the machine to ensure a large enough fraction of the power is radiated away close to the edge, to prevent excessive bombardment of the divertor and vessel wall by high energy particles, giving rise to release of Tungsten or vessel wall material and yielding uncontrolled increase of non-wanted impurity influx.

During the first DT campaign in JET, it was demonstrated experimentally that adding small amounts of ${ }^{3} \mathrm{He}$ to the discharge can help boost the neutron rate in the activated phase of the reactor. Although excessively expensive [6] and hence not a suitable candidate for routine operation, earlier modelling (see e.g. [7]) indicated that using this gas can be useful in the start-up phase of the discharge. So unless indicated otherwise, the computations in the present paper will be done assuming there is $3 \%$ of ${ }^{3} \mathrm{He}$ in the gas mixture. In the flat top phase, this concentration will fall to zero. ${ }^{3} \mathrm{He}$ also has a potential role to play in the early life of the machine, when the systems on the DEMO machine will be tested in absence of the actual gases required for ignition and when not all heating systems will yet be available at full power so that temperatures will be significantly more modest.

The present paper focuses on wave heating in the ion cyclotron resonance frequency range and only touches on other aspects when they are directly relevant to the ion heating discussion. More information on dedicated neutral beam injection and electron cyclotron heating can be found elsewhere (see e.g. [8] and the references therein). Transport is briefly discussed but a more thorough discussion of the competition between various transport mechanisms is not attempted.

Present-day estimates - somewhat differing depending on the assumptions made on the size and confinement properties of the machine - predict DEMO requires of the order of $100 \mathrm{MW}$ of auxiliary heating power to initiate the fusion fire. The auxiliary heating will likely be secured by a combination of heating methods. Compared to the fusion power the machine is projected to generate $(2 G W$, of which $20 \%$ is carried by the $\alpha$ 's), this is a fairly modest amount of power, underlining that the heating systems' key role in the ignited phase is not heating, the latter dominantly being provided by the fusion-born alphas. On the other hand, ohmic heating is insufficient to bring the DEMO plasma to ignition and the auxiliary heating systems are crucial to guarantee fusion relevant temperatures can be reached to initiate the burn.

\section{Basics of RF wave induced plasma heating}

The power absorbed density by a Maxwellian ion subpopulation is roughly given by [9]

$$
P_{a b s}=\frac{\omega_{p}^{2}}{16 \pi^{1 / 2}} \frac{\left(k_{\perp} \rho_{L}\right)^{2(N-1)}}{k_{/ /} v_{t h}} \frac{N}{(N-1) ! 2^{N-1}}\left|E_{+}\right|^{2} \exp \left[-\left(\frac{\omega-N \Omega}{k_{/ /} v_{t h}}\right)^{2}\right]
$$


in which $\omega_{p}$ is the plasma frequency, $\Omega$ the cyclotron frequency, $\rho_{L}$ the Larmor radius, $k_{/ /}$and $k_{\perp}$ are the parallel and perpendicular wave numbers w.r.t. the static magnetic field, respectively, $v_{t h}$ is the thermal velocity, $N$ the cyclotron harmonic and $E_{+}$the wave component rotating in the sense of the ions. The Gaussian factor on the right is maximal at the cyclotron layer $\omega=N \Omega$ and dies away when moving away from it, a spreading caused by the resonance $\omega=N \Omega+k_{/ /} v_{/ /}$being Doppler shifted away from the cold plasma resonance $\omega=N \Omega$ by finite temperature and/or macroscopic flow effects. On account of the fact that the power density is proportional to the density via the square of the plasma frequency and since $k_{\perp} \rho_{L}$ is small for the (fast magnetosonic) wave commonly relied on for achieving radio frequency heating, one historically (see e.g. [10]) first tested majority heating at $\omega=\Omega$. It was quickly realised that the presence of the plasma impacts strongly on the wave polarisation. In particular, when only 1 type of ions is present, $E_{+}$goes through zero exactly at the cyclotron layer, prohibiting to profit from the cyclotron resonance. Adding a second ion specie to the plasma allows to cure this.

A cold plasma approximation suffices to get a feeling of how the polarisation changes. In terms of the Stix coefficients $L$ (resonant at $\omega=\Omega), R$ and $S=(R+L) / 2$ the fast wave dispersion can be written as [11]

$$
k_{\perp, F W}^{2}=k_{o}^{2} \frac{\left[R-n_{/ /}^{2}\right]\left[L-n_{/ /}^{2}\right]}{\left[S-n_{/ /}^{2}\right]}
$$

where $k_{o}=\omega / c$ with $c$ the speed of light, and $n_{/ /}=k_{/ /} / k_{o}$. The fast wave is excited by a poloidal current on the antenna straps. Its cyclotron heating relevant polarisation is given by

$$
\frac{\left|E_{+}\right|}{\left|E_{-}\right|}=\frac{R-n_{/ /}^{2}}{L-n_{/ /}^{2}}
$$

where $E_{-}$is the polarisation component rotating in the sense of the electrons and $E_{+}$ the component rotating in the sense of the ions, or by

$$
\frac{\left|E_{+}\right|}{\left|E_{p o l}\right|}=\frac{R-n_{/ /}^{2}}{S-n_{/ /}^{2}}
$$

where $E_{p o l}$ is the poloidal electric field component. From these expressions it can be seen that the fast wave has a cut-off at $R=n_{/ /}^{2}$ and at $L=n_{/ /}^{2}$. The former occurs at low density (plasma edge) and is important for coupling while the latter occurs at high density (core plasma) and is important for heating. Moreover the wave has a resonance at $S=n_{/ /}^{2}$; in more sophisticated models adding finite temperature effects this resonance becomes a confluence with the ion Bernstein wave.

Glancing at the above expressions it becomes clear that the wave component responsible for ion heating is large at the high density cutoff as well as the confluence, allowing to render the wave absorption more potent when locating those layers close to but not at the cyclotron resonance. Perkins [12] has shown that the distance from the cyclotron resonance to the confluence-cutoff pair scales with the minority density so that adding a small minority to a majority plasma creates a confluence-cutoff pair close to the minority cyclotron layer. While $P_{a b s}$ now stays finite at the cyclotron layer, the Doppler broadening allows to optimally profit from the enhanced magnitude of $E_{+}$at 
the confluence-cutoff. Note that fundamental minority and second harmonic majority heating can take place at the same location when the respective charge-to-mass ratios appearing in the definition of the cyclotron resonance scale as 1:2. This effect is routinely exploited experimentally. Note as well that harmonic heating schemes benefit from low magnetic field strengths (the Larmor radius is inversely proportional to the static magnetic field strength), an asset of importance in the early testing phase of a machine when operated at reduced field. For completeness is should be mentioned that the above expression for the absorbed power density is valid for not too energetic particles; contributions due to $E_{-}$are to be added for fast ion populations.

The usual minority heating scheme has a disadvantage: increasing the minority concentration enhances the $\omega_{p}^{2}$ factor but equally moves the confluence-cutoff away from the cold cyclotron resonance. As a result minority heating typically grows roughly linearly at small concentrations but then exhibits a roll-over and results in dwindling ion damping at further increased concentrations. Kazakov proposed to add a third ion specie to the mix. It was proven theoretically as well as experimentally that this allows to achieve very significant absorption of minute amounts of minority ions [13, 14]. This scheme allows to further boost the optimisation by adding an extra free parameter: with 3 ions being present, the confluence-cutoff pair of the main 2 ion species can be moved at will between the cyclotron resonances of these species upon changing their relative concentrations. If the cyclotron layer of a third species lies in between that of the main $2\left([Z / A]_{1}<[Z / A]_{3}<[Z / A]_{2}\right)$, the confluence-cutoff allowing enhanced absorption can be aligned with the region where the absorption by a third ion specie is maximal.

\section{$3 \quad$ ICRH tasks}

Ion cyclotron resonance heating can provide assistance in all phases of a DEMO discharge. Various tasks it can (help) perform will be dealt with in somewhat bigger detail in later sections but here is a list and a very brief description (see Fig. 1):

- Start-up: Ample experimental evidence shows that the presence of RF fields at modest power allows to initiate a plasma at lower induced loop voltage.

- Ramp-up: During the ramp-up phase, the density and temperature are gradually climbing towards fusion-relevant values. In that phase of the discharge various heating methods are not yet at their best performance: neutral beam injection at the energy level required for operation in the active phase cannot be used because of excessive shine-through, and wave heating methods - both ion and electron heating applications - have limited performance because the absorption efficiency scales with the density. Moreover, cyclotron harmonic heating effects $(N>1)$ are not efficient because they require preheating. Finally, the current still being ramped up - at least in the very early phase of the discharge - yields poor fast ion confinement and thus risk of excessive bombardment of the vessel wall by fast particles escaping from the main plasma. In this phase, ion cyclotron heating at the fundamental cyclotron frequency can be of assistance to help crank up the plasma temperature and to keep the temperature peaked, avoiding impurity accummulation. This type of heating remains efficient at modest temperature and affects the whole distribution function so that - for a prescribed power density - 
more modest fast particle tails are being formed than is the case for harmonic heating schemes.

- Ignited phase: In the main phase of the discharge when the densities and temperatures have reached their nominal operation level, heating mechanisms are typically at their peak performance. As will be illustrated later, this is not uniformly beneficial: in large, hot, dense plasmas the waves tend to lose a significant fraction of their power before they reach the plasma core. Depending on the exact parameters (plasma composition, magnetic field strength, presence/absence of fast particle subpopulations) different heating schemes dominate. As long as fusion reactions are not occurring, the heating methods fulfil their primarily role: ensuring fusion-relevant temperatures can be reached. Once ignited, the plasma is dominantly heated by fusion-born alpha particles Coulomb collisionally slowing down from their birth energy of $3.5 \mathrm{MeV}$, the available heating methods are no longer needed to guarantee their primary task and can be exploited for other tasks such as current drive and impurity removal. ICRH can also be of use for burn control (see e.g. $[15,16]$ ), by influencing the MHD behaviour via fast ion populations or - more simply - by adjusting the operation temperature.

- Landing: Experimental evidence in metal wall machines shows that it is delicate to "land" high performance shots, the reason being that particle and energy dynamics are associated with slightly different confinement times which can result in a more swift temperature than density drop, causing increased impurity influx, potentially even making the temperature profile hollow, and yielding a thermal quench disrupting the plasma. The high performance regime is characterised by high temperatures and densities, requiring high currents to ensure good confinement. As disruptions at high current can have devastating effects on the machine, methods that ensure a smooth falling out of the deep H-mode regime are required for safe operation. As ICRH waves can carry power to the plasma core when properly choosing the operation frequency, this heating method allows keeping the temperatures profiles peaked.

- Wall conditioning: The first wall of DEMO is a challenge for material research. The wall conditioning requirements as well as the plasma facing materials, operated at unprecedented temperatures $\left(>400^{\circ} \mathrm{C}\right)$, are at present undecided. Retention of radioactive tritons are a particular concern, to keep self-fuel-sufficiency and simultaneously ensure tritium safety. Among the methods to ensure wall cleaning in presence of a magnetic field, ICRH - allowing to affect both ions and electron dynamics - is a prominent one.

\section{Preliminary notes: tools and approximations}

From the wave's point of view, radio frequency heating is the process of energy being launched into the vessel via an antenna and being lost to the plasma. From the particles' vantage point, it is the process of net acceleration brought about by energy imparted to various populations via electromagnetic waves and either passed on to other species via Coulomb collisions or lost. The former dynamics is described by the wave equation while the latter requires solving the Fokker-Planck equation. A few 


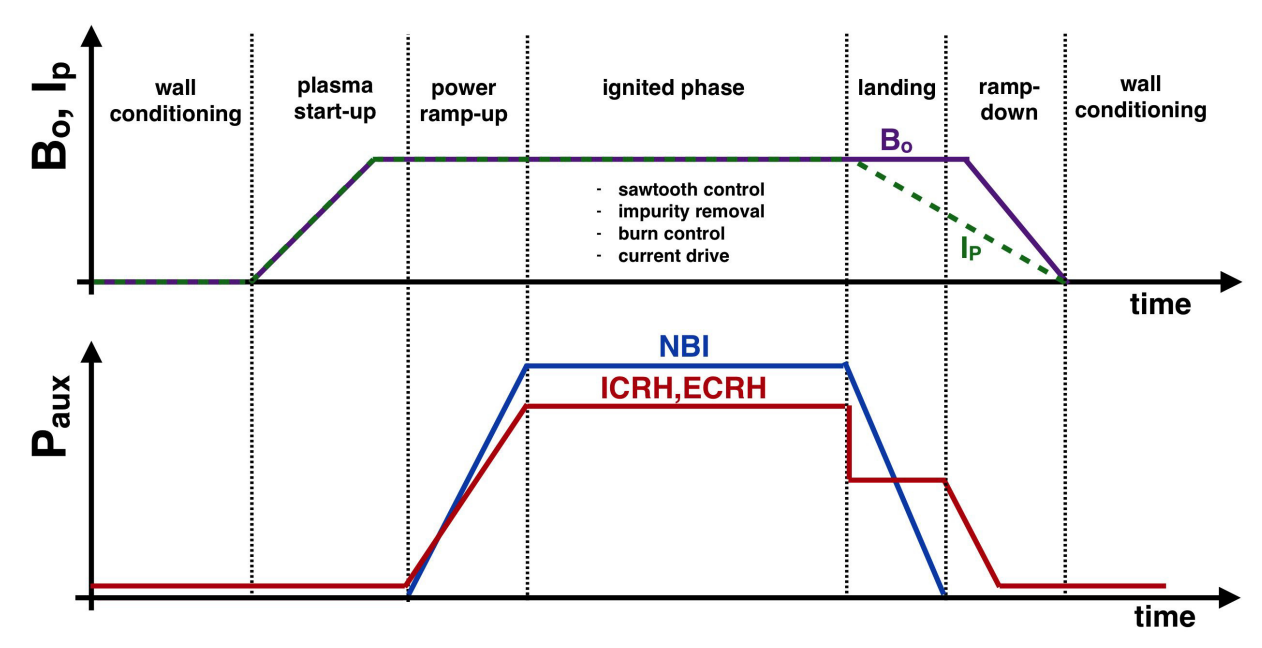

Figure 1: Tasks the ICRH system can fulfil during a DEMO shot.

notes on the adopted wave and Fokker-Planck solvers can be found in Appendices A $\&$ B. The tool used for giving a crude assessment of the transport is discussed in Appendix C. A brief discussion of the orbits and their impact on the results is reserved to Appendix D. The fusion born alpha particle distribution is illustrated in Appendix E. Finally, a justification of the equivalent temperatures used for modelling high energy populations in the wave equation solver is provided in Appendix F.

\section{$5 \quad$ Radio frequency heating scenarios at full field}

\subsection{Ion cyclotron positions as function of the frequency}

Figure 2 depicts the position of the cyclotron layers as a function of the antenna frequency for the adopted reference $B_{o}$ strength, 5.855T. At very low frequencies, there are no ion cyclotron layers of the fusion fuel ions $\mathrm{D}$ or $\mathrm{T}$ inside the plasma. Core heating of one of these ions requires a frequency of $\approx 30 \mathrm{MHz}$ for fundamental cyclotron $T$ heating and $\approx 45 \mathrm{MHz}$ for $\mathrm{D}$ heating. Core ion heating relying on ${ }^{3} \mathrm{He}$ requires $f \approx 60 \mathrm{MHz}$ but requires collisional relaxation of the ${ }^{3} \mathrm{He}$ on the bulk to initiate $D-T$ fusion. The fundamental cyclotron layer of ${ }^{3} \mathrm{He}$ coincides with the second harmonic layer of $T$ so direct heating of one of the fuel ions is equally possible at $60 \mathrm{MHz}$. The next window of opportunity lies around $90 \mathrm{MHz}$ for which the second harmonic layer of $D$ lies in the centre.

\section{2 'Classical' scenarios}

In Fig. 3 the absorption efficiency - as predicted by the TOMCAT $1 \mathrm{D}$ wave equation solver - is plotted for $n_{\text {tor }}=50$. Up to a few exceptions at fairly low frequencies full single pass absorption is achieved. The windows sketched in the previous subsection are clearly visible. The abrupt changes of the type of absorption slightly under $30 \mathrm{MHz}$, at $45 \mathrm{MHz}$, somewhat under $70 \mathrm{MHz}$ and again at $\approx 90 \mathrm{MHz}$ are the consequence of 

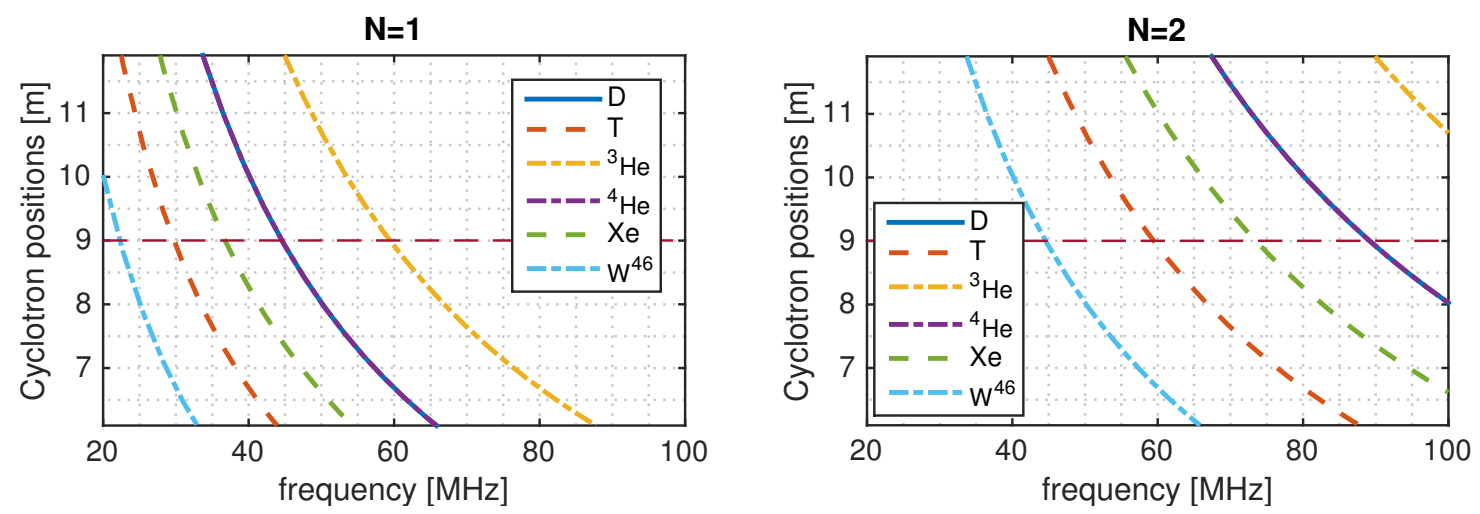

Figure 2: Cyclotron layers for fundamental cyclotron heating (left) and second harmonic heating (right) as a function of the frequency.

cyclotron layers entering the machine at the low field side, blocking the $R F$ power. Moreover, cyclotron resonances close to the antenna hold the risk of particle acceleration in the edge, leading to particles bombarding the wall and hence to sputtering and to wall particles entering the main plasma. So, although e.g. the frequency of $47 \mathrm{MHz}$ seems to be optimal for ${ }^{3} \mathrm{He}$, it actually corresponds to far off-axis heating and is therefore not very desirable. Note that in the window were $D$ heating is possible, ${ }^{4} \mathrm{He}$ unavoidable occurs as well: $\mathrm{D}$ and ${ }^{4} \mathrm{He}$ have the same charge over mass ratio and thus their cyclotron frequency is identical. In spite of the fact that they are not very abundant, the fast - fusion born - alpha particles absorb more power than both the thermalised ${ }^{4} \mathrm{He}$ and the $\mathrm{D}$ majority. The deleterious impact of the alpha particle absorption (particles with already high energies and therefore less subject to Coulomb collisions than colder ones) get further accelerated to even higher energies and become even less collisional, contributing less ideally to the fuel ion heating; remind $\alpha$ particle heating via alpha particles slowing down on the bulk populations is intended to be the main heating scheme in the ignited plasma. For that reason, $\alpha$ particle heating is commonly avoided in fusion machines.

Although the antenna frequencies are chosen to favour ion heating, electron heating often dominates the ion heating. This is an unavoidable side effect of large size, hot, dense machines: whereas ion cyclotron heating occurs close to cyclotron layers and fades quickly when going away from the cyclotron resonance layer in the RF domain of frequencies, electron damping and transit time magnetic pumping are not bound to a cyclotron layer. They merely require the thermal velocity of the electrons to crudely match the parallel phase velocity of the wave, a condition commonly more easily satisfied than the cyclotron resonance. Hence, in hot, dense machines waves tend to lose energy to electrons everywhere on their path to the cyclotron layer. And as machines such as DEMO are large, the fraction of power lost before even reaching the first ion cyclotron layer is significant. As a consequence, determining the optimal frequency for ion heating is a trade-off between losing power to the electrons to allow reaching higher power densities in the centre and obtaining higher ion damping at the price of not reaching the actual centre. This disadvantage is actually an advantage when current drive rather than heating is intended (see further): due to the fact that ion and electron heating can be exchanged via a small change in frequency, the bandwidth 
required to allow both heating and current drive to be possible is well within reach of what technologically is possible (see [17] showing good antenna-plasma coupling performance can be guaranteed in a region of $\approx 10 \mathrm{MHz}$ width). On the other hand, it makes it hard to concentrate power inside the $q=1$ layer, a condition generally thought to be a prerequisite for building a peaked temperature profile allowing chasing high $\mathrm{Z}$ particles from the core by either reverting the neoclassical pinch [18] or by relying on fast particle pressure in the core [19]. Figure 4 shows the cumulative power deposition profiles as a function of the flux surface labeling parameter ( $\rho$ is half the diameter of the local magnetic surface in the midplane) for 3 different frequencies as predicted by a $2 \mathrm{D}$ wave equation solver; the positions of the relevant cyclotron layers are depicted in Fig. 5. The lowest $(f=50 \mathrm{MHz})$ results in dominant ion heating but necessitates ion heating fairly far from the core. Adopting $f=60 \mathrm{M} \mathrm{Hz}$ yields central ion heating (all ion heating takes places maximally $30 \mathrm{~cm}$ from the magnetic axis, the physical width of the absorption being $\approx 60 \mathrm{~cm}$ symmetrically spread around the axis) but results in electron heating dominating ion heating. The last places the ${ }^{3} \mathrm{He}$ and $T$ cyclotron layers on the high field side and yields dominant majority $\mathrm{D}$ and alpha particle heating.

The plot for the non-activated phase (where the fast as well as slow ${ }^{4} \mathrm{He}$ population is absent) is extremely similar to that in Fig. 3 except for the fact that in the frequency intervals where $D$ can absorb one now gets enhanced $D$ bulk and $D$ beam heating (plot not shown). Similarly, in absence of ${ }^{3} \mathrm{He} N=2 \mathrm{~T}$ heating substitutes ${ }^{3} \mathrm{He}$ minority heating $\left({ }^{3} \mathrm{He} N=1\right.$ heating serving as "pre-heating" to allow $N=2 \mathrm{~T}$ heating to take over more easily) when ${ }^{3} \mathrm{He}$ is no longer used (corresponding plot not shown either).

Similar to what was found in earlier studies - and the solution adopted for ITER - one concludes that $N=2 T$ heating is the preferred ICRH heating scheme for the DEMO reactor in its activated phase. It does not suffer from spurious alpha particle or $D$ heating, except when heating too far on the high field side. And a window where electron heating is dominant (a prerequisite for current drive) is available at a nearby frequency. Aiming at a bandwidth from $f=55$ to $65 \mathrm{MHz}$ seems ideal for designing an antenna system.

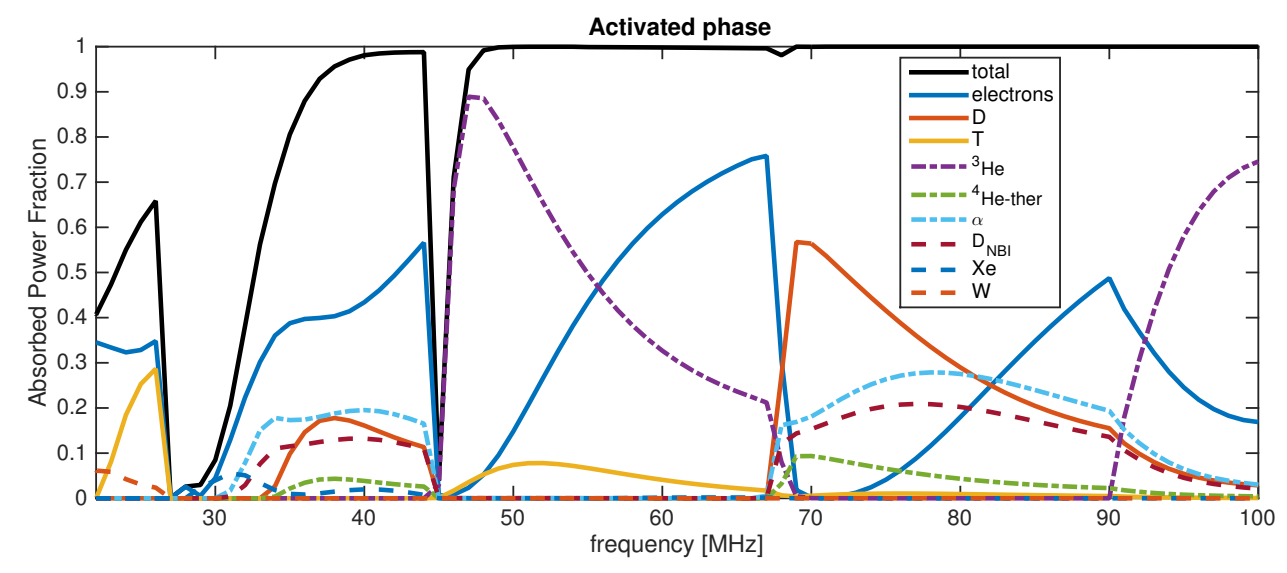

Figure 3: Absorption efficiency as a function of the frequency.

Figure 6 depicts the heating efficiency as a function of the toroidal mode number (top), the core temperature (middle) and core density (bottom) for a driver frequency 

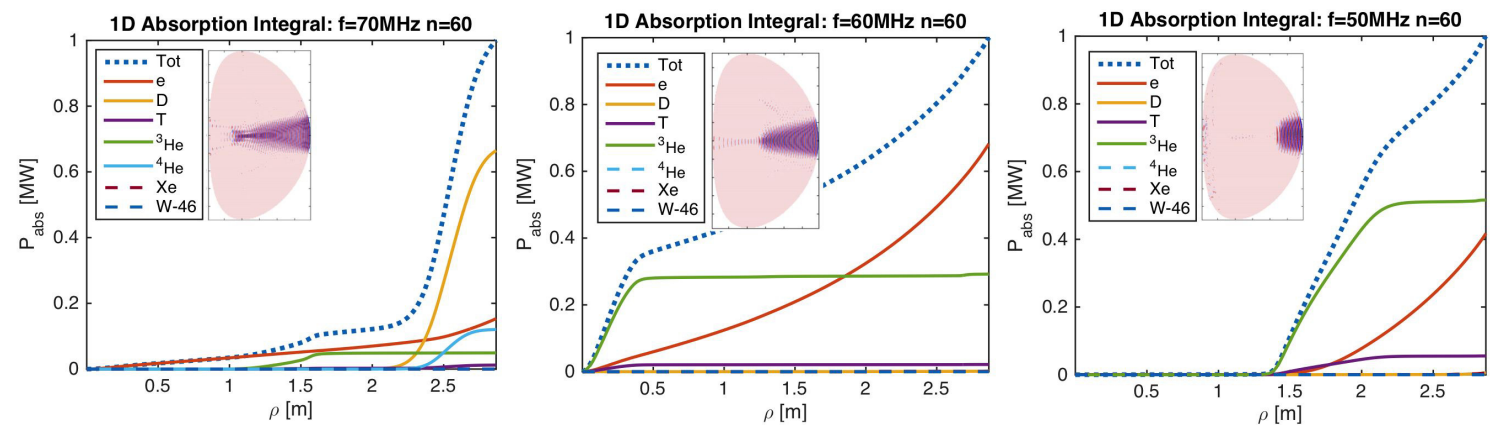

Figure 4: Cumulative power deposition profile as obtained by solving the 2D wave equation for low field side (left; $f=70 \mathrm{MHz}$ ), core (middle; $f=60 \mathrm{MHz}$ ) and high field side (right; $f=50 \mathrm{MHz}$ ) heating. The bottom right plot provides the cyclotron positions in the equatorial plane.

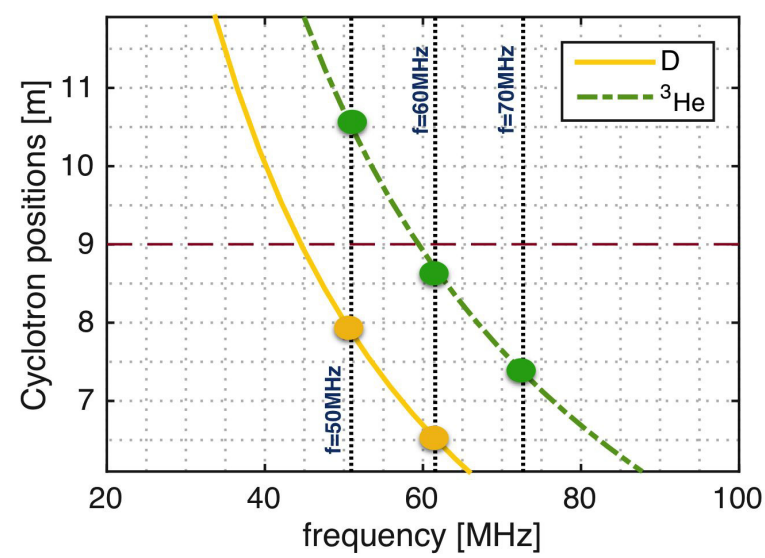

Figure 5: Cyclotron positions corresponding to the absorption profiles depicted in Fig. 4.

of $60 \mathrm{MHz}$ so that core ion heating is ensured. It shows that a good absorption efficiency is always guaranteed for physically relevant parameters: (i) The antenna should be designed avoiding co-axial modes [20] since these modes carry power around the machine in the low density edge region rather than taking it to the core, enhancing the risk for RF induced sputtering. But one should also avoid a too wide evanescence between the antenna and the last closed flux surface (too high $k_{/ /}$) to avoid needing high amplitude evanescent waves to guarantee the coupling. The vacuum wave number being $k_{0}=\omega / c=1.2 / m$ ( $\omega=2 \pi f$ and $c$ is the light speed) choosing $k_{/ / \text {,antenna }}$ to be larger than but of the order of $k_{0}$ i.e. $n_{\text {tor }}=k_{/ / \text {,antenna }} R_{\text {antenna }} \approx 25$ would be suitable (TOMCAT ignores the presence of the poloidal field and hence $k_{/ /}$is identified with $\left.k_{\text {toroidal }}\right)$. For these values, the absorption efficiency is higher than $80 \%$ and one is close to the position where ion heating is optimised. As will be discussed in a dedicated session, somewhat higher toroidal mode numbers $\left(n_{\text {tor }} \approx 50-60\right)$ are characteristic for the traveling wave antenna proposed for DEMO. (ii) Minority heating is already efficient at temperatures well below those reached in the main operation phase. The total single transit absorption increases while the temperature builds up; near-total absorption is achieved for temperatures beyond $10 \mathrm{keV}$. Approaching temperatures relevant for the 
burn phase, electron heating gradually increases. At that time, excellent absorption is guaranteed and high density can be reached to enable equipartition to help further increasing the ion temperature. But it remains a fact that in large size machines electron heating tends to outperform core ion heating. Peaked temperature profiles counteract this effect and favour ion heating: edge regions remain fairly cold so that the condition for optimal electron heating is not satisfied, while core regions benefit from direct and indirect (Coulomb collisional) heating. Provided enough power is available - required for ensuring high $\mathrm{Z}$ impurity chase-out and one of the intended tasks of RF heating (see further) - this process can be self-enforcing. Wave induced core ion heating profiles are typically peaked while those due to neutral beam injection tend to be wider; a dedicated transport study is required to decently answer the question whether the proper core conditions needed for high- $Z$ chase-out can be realised. Although a full transport study is outside the scope of this paper, a first tentative image is provided in a dedicated section.

Figure 7 shows the absorbed power as a function of the ${ }^{3} \mathrm{He}$ concentration when $f=60 \mathrm{MHz}$ and $n_{\text {tor }}=30$ or 60 early in the shot $\left(T_{o}=14 \mathrm{keV}\right)$ and when the operation temperature has been reached $\left(T_{o}=28 \mathrm{keV}\right)$. Almost $100 \%$ of the power is absorbed in a single pass over the plasma when the desired operational temperature has been reached. The electron damping is typically $60-70 \%$ while the ion heating changes from modest second harmonic heating of the $T$ majority to fundamental cyclotron heating of a small ${ }^{3} \mathrm{He}$ minority. The ${ }^{3} \mathrm{He}$ heating exceeds that of the majority when $X\left[{ }^{3} \mathrm{He}\right]>0.5 \%$. At $\mathrm{T}_{o}=28 \mathrm{keV}$ a shallow maximum ${ }^{3} \mathrm{He}$ single pass absorption of $30 \%$ is reached between 2 and $7 \%$. As can be seen in the top subplots, the use of ${ }^{3} \mathrm{He}$ is particularly suited during the ramp-up phase, when the temperature is still climbing to its flat top value, the maximum being even more pronounced at low toroidal mode numbers. Operating at modest ${ }^{3} \mathrm{He}$ concentration $(\approx 2-3 \%)$ is suitable, ensuring strong single pass damping. When the temperature approaches its flat top value, the electron heating has strongly increased. Somewhat surprisingly, the second harmonic heating of tritium is about equally strong in the early phase as in the later phase of the discharge, despite the fact that $N=2$ heating scales with temperature. This is due to the electron damping having increased so that less power reaches the core when the temperature is higher. As electron temperature peaking is thought to be beneficial for impurity chase-out, not using ${ }^{3} \mathrm{He}$ in the main plasma phase not only has the advantage of being cost efficient $\left({ }^{3} \mathrm{He}\right.$ being expensive) but also of allowing to form high energy tails dominantly slowing down on electrons.

Plasma constituents that are directly heated by the RF waves form high energy tails and heat the other particles while Coulomb collisionally slowing down. In the presence of a ${ }^{3} \mathrm{He}$ minority, both the minority and the $T$ majority are heated. Assuming a total power density of $P_{R F}=0.5 \mathrm{MW} / \mathrm{m}^{3}$ as well as a local core heating power fraction obtained from wave equation using the main $\left(N=1{ }^{3} H e, N=2 T\right)$ heating scenario in presence of ${ }^{3} \mathrm{He}(17 \%$ going to the electrons, $8 \%$ going to the tritons and $75 \%$ to the ${ }^{3} \mathrm{He}$ minority), the minority effective temperature $T_{\text {eff }}=3 / 2<N k T>$ (where $\langle\ldots\rangle$ denotes the average over velocity space) is kicked up to almost $70 \mathrm{keV}$ while the tritons and deuterons temperatures increase by about $10 \mathrm{keV}$; see the drawings in Fig. 8 depicting the local energy density. When the plasma has been heated sufficiently, the ${ }^{3} \mathrm{He}$ gas valve is closed and only the $\mathrm{T}$ ions are directly heated. Typical numbers for this case are $27 \%$ going to the electrons and $73 \%$ to the $T$ majority. Because 

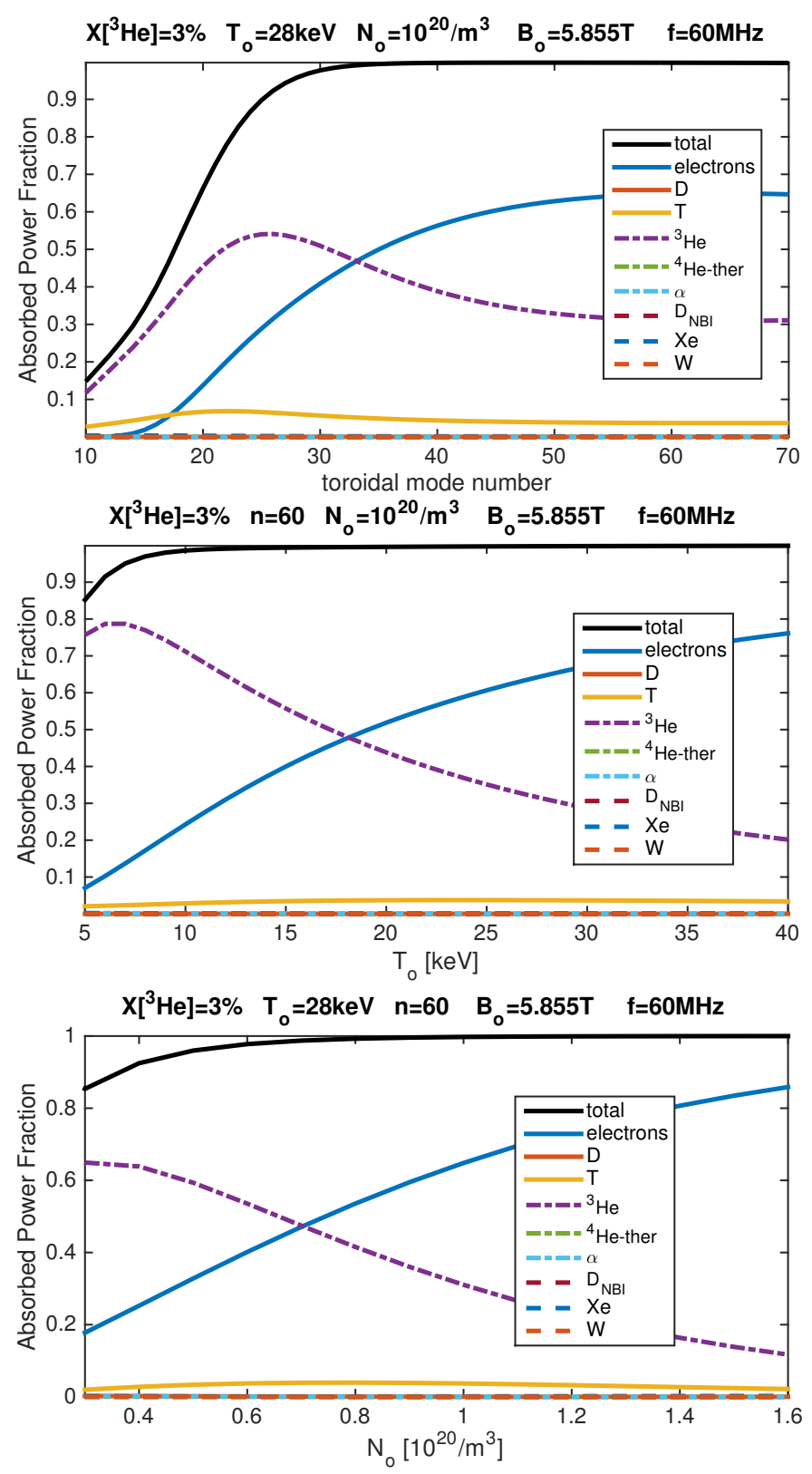

Figure 6: Absorption efficiency as a function of the toroidal mode number (top), core temperature (middle) and density (bottom) for 60MHz.

the $T$ majority is heated at its second harmonic cyclotron frequency, most of the particles of the thermal population do not significantly gain energy so that the effective temperature is only increased to $\approx 50 \mathrm{keV}$. A very high energy tail containing only a small fraction of the particles is formed; see the drawings in Fig.9.

It is important to underline that these temperatures are not the ones that will ultimately be achieved: via spatial diffusion energy is evacuated from regions where it was deposited by the waves or passed on via Coulomb collisions, yielding temperature profiles that are broader but less high. A detailed study incorporating the effects 

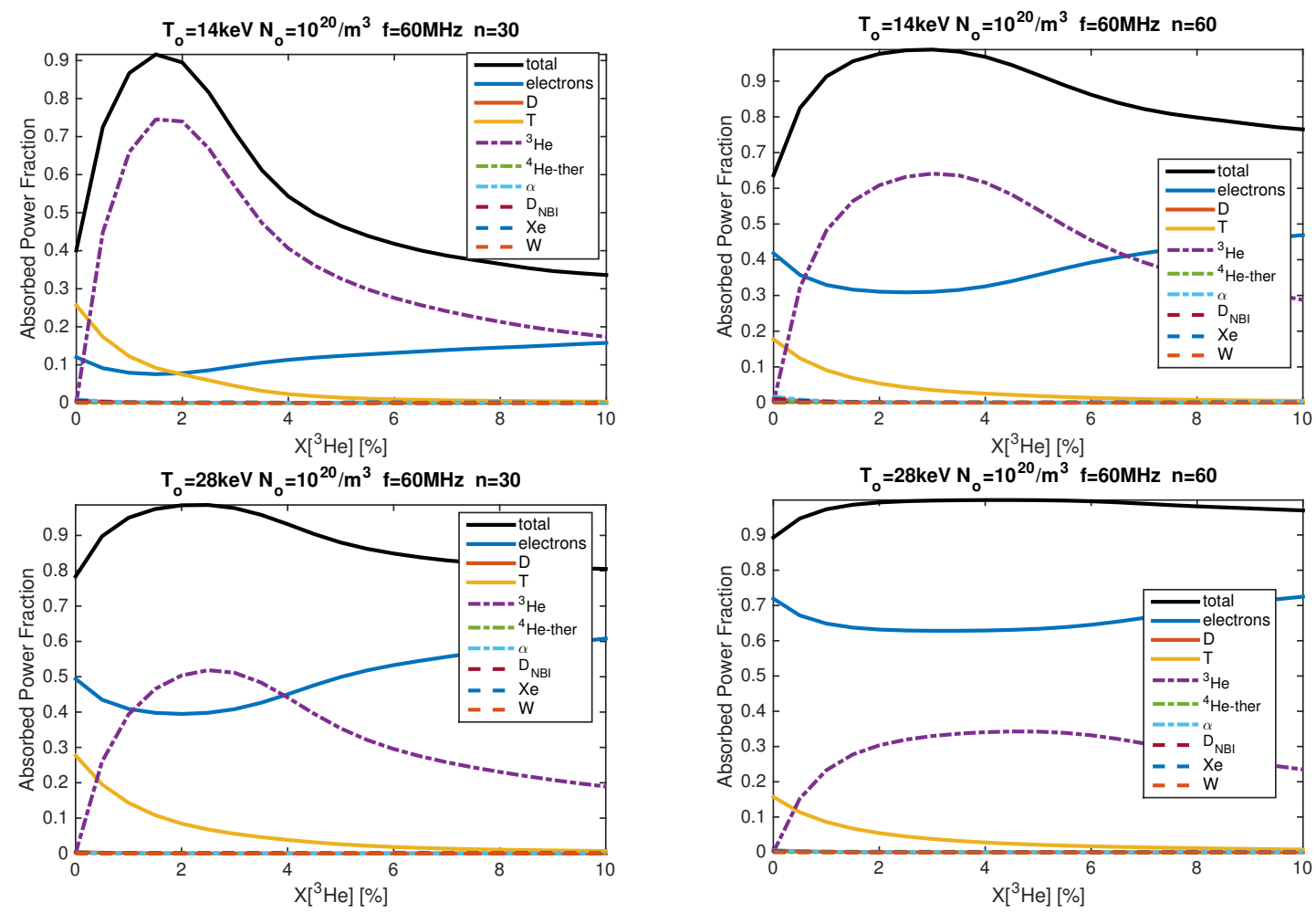

Figure 7: Absorption efficiency as a function of ${ }^{3} \mathrm{He}$ concentration when $f=60 \mathrm{MHz}$ and $n_{\text {tor }}=$ 30 (left) or 60 (right) during the temperature ramp-up (top) and the early steady state phase (bottom).

of transport is beyond the present study but is essential to assess the potential of a heating scheme; a crude assessment using a simplified transport model is provided in a separate section. The energy sources required as input for such a transport model are the powers after Coulomb collisional redistribution. Figure 10 depicts the fraction the power passed on from the ${ }^{3} \mathrm{He}$ minority or the $\mathrm{T}$ majority for various power densities and minority concentrations. When the power density is modest, only weak tails are formed, so that most of the particles have energies below the critical velocity yielding dominant indirect ion heating. Efficient indirect electron heating requires a significant power density. Note that fundamental cyclotron ${ }^{3} \mathrm{He}$ minority heating at very low concentration (1\%) and second harmonic majority $T$ perform similarly; in both cases most of the energy of the distribution is carried by a small fraction of very energetic particles. The potential of the minority to heat the electrons (useful for ensuring a peaked electron temperature profile and currently thought to be a requisite for impurity chasing) disappears rapidly when the minority concentration increases: at concentrations of $3 \%$ it requires power densities in excess of $1 \mathrm{MW} / \mathrm{m}^{3}$ to pass more power to the electrons than to the ions. Second harmonic heating much more efficiently heats the electrons at such power levels in DEMO. To simultaneously guarantee bulk ion heating while ensuring impurities cannot flood the core, a small but finite minority concentration seems beneficial. In the very early phase of the temperature rampup when second harmonic heating is not yet efficient while fundamental heating is, 

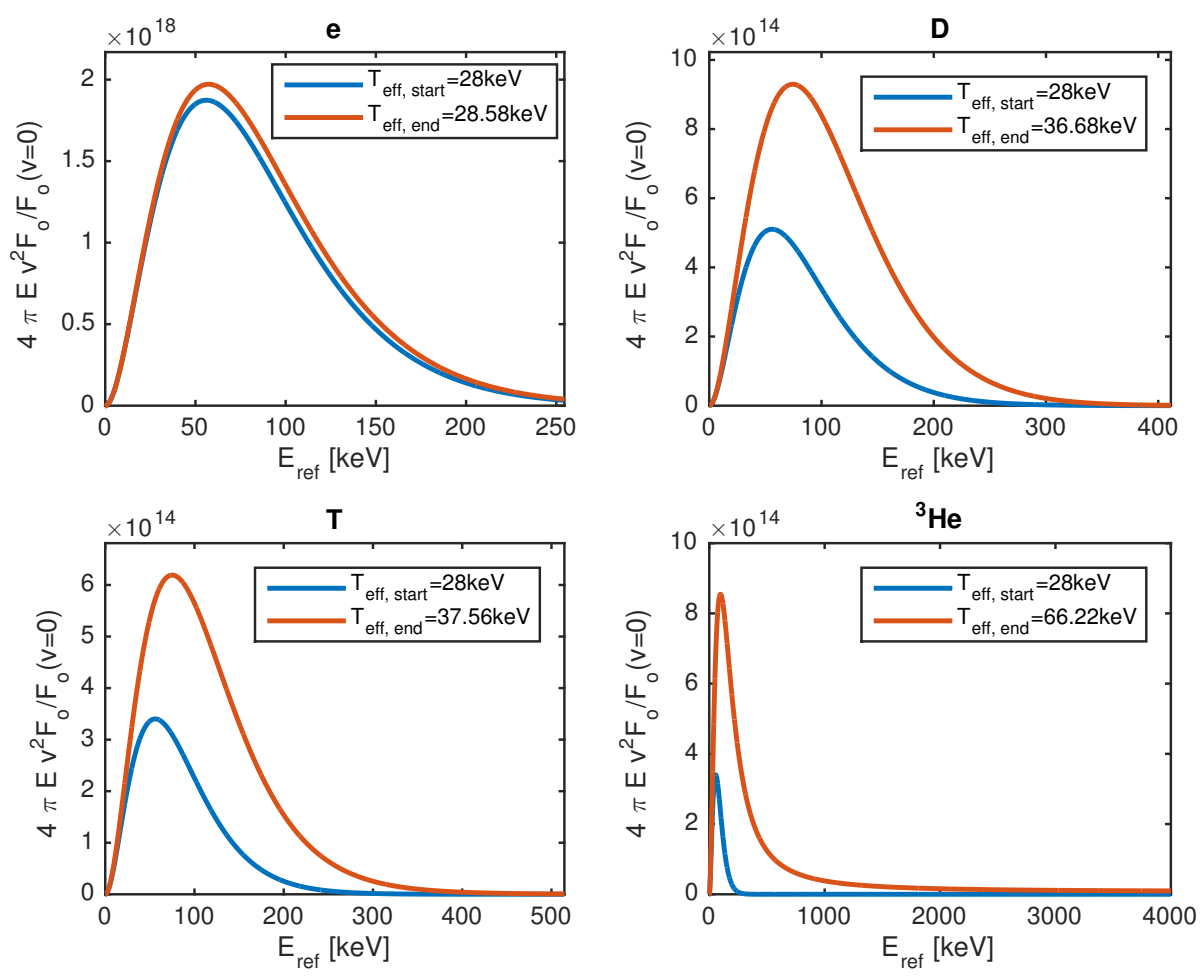

Figure 8: Local energy densities $4 \pi E F_{o} v^{2}$ at the peak absorption point of the various ion constituents of a $D-T$ plasma with a ${ }^{3} \mathrm{He}$ minority concentration.

minority heating is the key RF heater. When reaching fusion relevant temperatures, $T$ gradually takes over the role of the minority. Operating at as modest as possible minority concentration is also beneficial for a more fundamental reason: as the fusion reactivity scales with the product of the fusion products, any non-fuel-ion dilutes the plasma and compromises the fusion yield. Operating at $3 \%$ of ${ }^{3} \mathrm{He}$ reduces the fusion yield with $10 \%$ and is equivalent to a non-balanced $65 \%-35 \%$ fuel mix. Operating at $5 \%$ decreases the reactivity by another $10 \%$, equivalent to an imbalance of $70 \%-30 \%$. Interpreted in the opposite direction, operating at lower minority concentrations allows to consume less $T$ fuel.

When accounting for the tail temperatures reached, the fraction of the power absorbed by the ions increases and dominates that absorbed by the electrons, independent of whether the increased $T$ temperatures have been accounted for. Figure 11 depicts the results when both tail temperatures have been accounted for; solely accounting for the increase minority temperature yields a very similar figure, except that the $T$ majority heating is now reduced by a fraction of $25 \%$, yielding a maximal absorption of about $30 \%$.

\subsection{Potentially supplementary options at full field}

The 3 -ion scheme (see e.g. $[13,14]$ ) exploits the freedom to dose the presence of 2 main ions to force the polarisation to be optimal at the cyclotron layer of a third ion. 

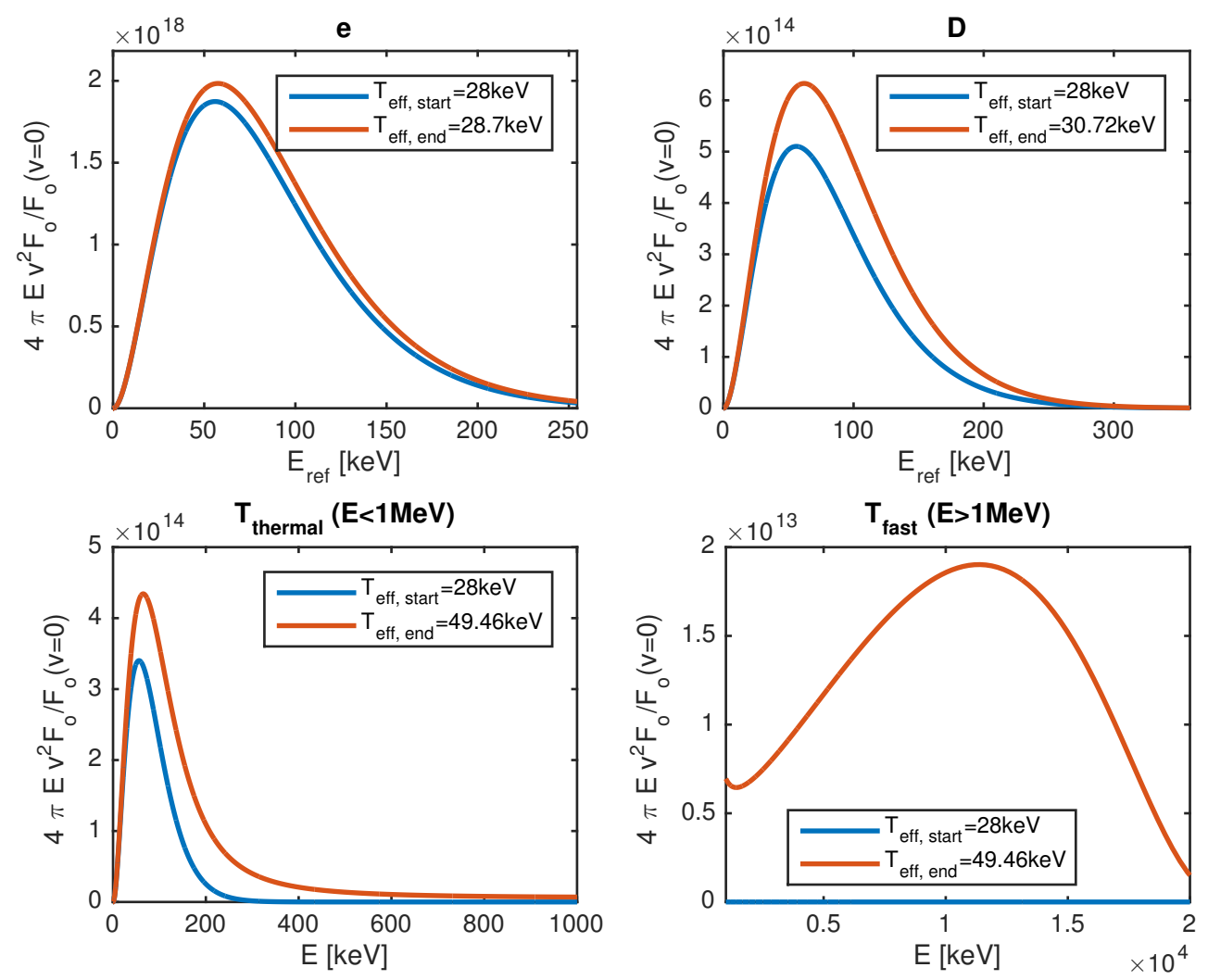

Figure 9: Local energy densities $4 \pi E F_{o} v^{2}$ at the peak absorption point of the various ion constituents of a $D-T$ plasma without a ${ }^{3} \mathrm{He}$ minority concentration. The 2 bottom plots depict the $T$ distributions, the left subplot focussing on the thermal and the right subplot on the fast subpopulation.

To illustrate the potential and particularities of this heating scheme, Fig. 12 shows the absorption efficiency of a $D-T$ plasma to which $0.1 \%$ of $A r$ (likely to be used in DEMO for the creation of a radiative belt in the outer layers of the plasma to avoid excessive power loads on the $W$ divertor) has been added to the ion mix and where the $\mathrm{T}$ fusion fuel concentration was varied during the ramp-up phase, adopting a central temperature of $15 \mathrm{keV}$ and omitting the fusion-born ${ }^{4} \mathrm{He}$. To allow an as wide as possible scan, the concentrations of the $\mathrm{D}$ beam has also been reduced to zero. At very low $X[T]$, the usual minority heating takes places, $T$ being the minority absorbing most of the power. At the other extreme, minority $D$ heating is dominant in a $T$-rich plasma. Recall that adding the fusion born alpha particles to the mix would degrade the $D$ absorption and replace it by non-desired $\alpha$ particle heating. The maximal $D$ absorption now drops from $90 \%$ to $50 \%$ (not shown), making the 3-ion scheme an ideal scheme for the start-up phase but not for the main burning phase, unless dedicated alpha particle heating is desired (see the current drive section). In the high temperature activated phase, the $D$ heating further degrades to $35 \%$, while electron heating becomes the dominant heating mechanism (not shown). Since adding 

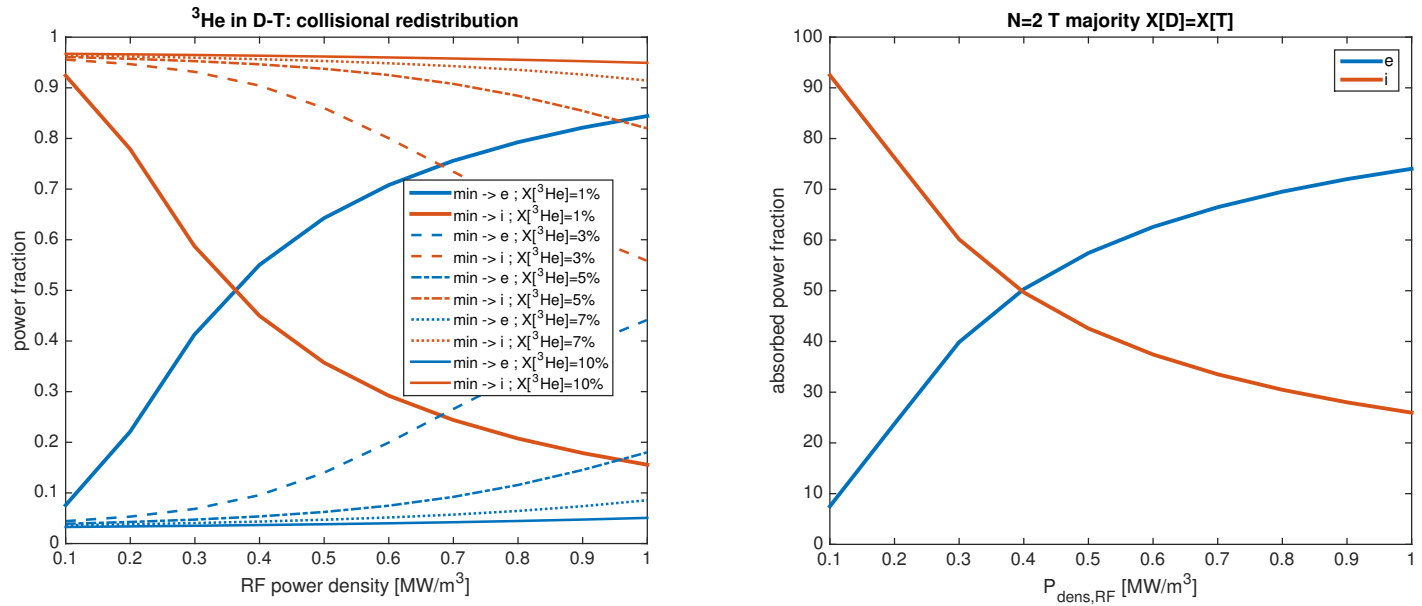

Figure 10: Fraction of the power directly absorbed by the ${ }^{3} \mathrm{He}$ minority (left) and the $\mathrm{T}$ majority (right) collisionally passed on to the electrons and the ions for various power densities. Various ${ }^{3} \mathrm{He}$ concentrations were considered for the left subplot.

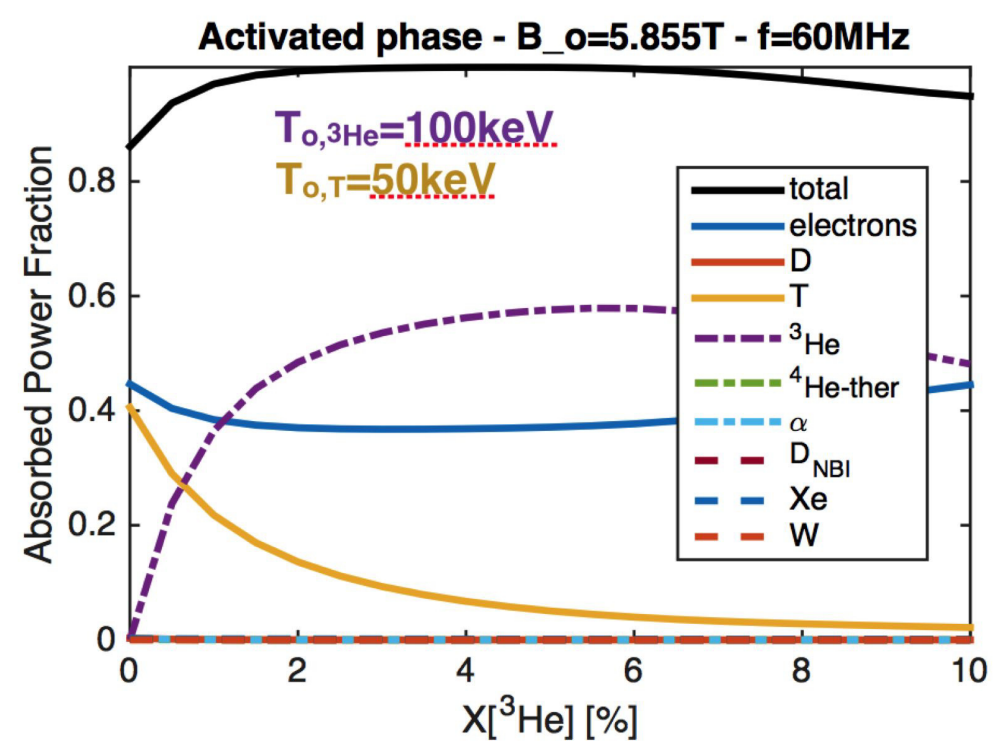

Figure 11: Single pass absorption when accounting for the ${ }^{3} H e$ and $T$ tails by adopting the appropriate effective temperatures.

an extra ion species to the mix introduces a supplementary ion-ion hybrid resonance, the absorption of an $A r$ minority as predicted by TOMCAT is very sensitive to the majority concentration mix: an incoming wave is reflected from the $D-T L$-cutoff and causes constructive or destructive interference at the cyclotron position of the $A r$ minority. Hence the $A r$ absorption oscillates as a function of the $T$ concentration. The optimal concentration occurs at nearly balanced D-T concentrations $(X[T]=53 \%)$ at which full single pass absorption is achieved. As long as the cutoff lies on the high field 
side of the $A r$ cyclotron layer, the interference pattern is due to the interaction of a fast wave approaching the cutoff and the wave reflected from it. When it crosses the cyclotron layer, the interference pattern also shows evidence of the shorter wavelength Bernstein wave branch. Ultimately, the cyclotron layer is screened and can no longer be reached, the $A r$ damping dropping to zero. The impact of such constructive/destructive interference was noticed first by Fuchs et al. as a means to crank up the absorption efficiency by profiting from the high field side $R$-cutoff [21]. Fuchs used a fast-waveonly non-kinetic model in which the power was lost at the artificial fast wave resonance rather than being lost to electrons or ions (respectively close to the conversion layer or to the cyclotron layer in a more sophisticated model). The importance of the position of fast wave cutoffs and their impact on the absorption efficiency was only gradually realised. Mayoral was the first to notice that the presence of $C$ in the discharge when operating JET with $C$ walls - $D$ having the same $Z / A$ as $D$ - prevented exploiting minority $D$ heating in a $H$ plasma since the parasitic $C$ raised the effective concentration of $D$-like particles beyond that which allowed minority $D$ heating in the inverted $(D)-H$ scenario, shifting the confluence layer and closing the gap of opportunity for minority heating [22]. Later JET work [23], confirmed the impact of plasma composition and the ensuing presence of multiple cutoff-confluence pairs in the plasma and their impact on absorption efficiency. Kazakov proposed to purposely steer the position of confluence/cutoff position of 2 main ions at the benefit of a third ion. Figure 12 - produced with a 1D wave equation solver and using a single toroidal mode number - over-accentuates the importance of the interference since the confluence position is $k_{/ /}$-dependent and the antenna excites a full spectrum of modes at differing parallel wave numbers. Moreover, a supplementary interference resulting from localised damping further attenuates the extrema.

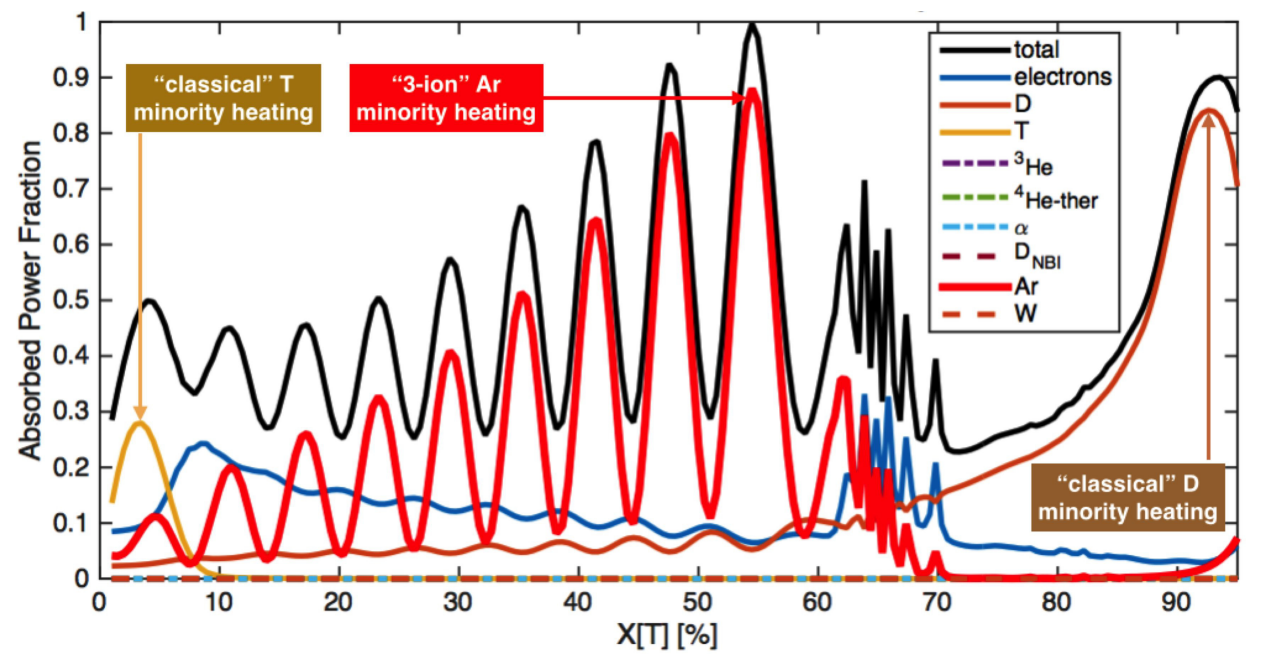

Figure 12: Absorption efficiency as a function of the $T$ concentration when $f=40 \mathrm{MHz}, n_{\text {tor }}=50$ and $T_{o}=15 \mathrm{keV}$.

Any third species that satisfies $[Z / A]_{1}<[Z / A]_{3}<[Z / A]_{2}$ for 2 species of which the relative concentrations can be varied is a possible candidate for 3 -ion heating. Depending on the actual $[Z / A]_{3}$, the relative concentrations of the other 2 species differs. For the adopted parameters, Beryllium, Lithium-7 and Argon roughly have 
their optimal operation point around balanced D-T concentrations. Note, however, that the frequency at which the 3 -ion scheme is active is outside the bandwidth possible for the $\mathrm{T}$ and ${ }^{3} \mathrm{He}$ ion so that it requires a dedicated antenna.

Whereas standard schemes perform poorly at modest $k_{/ /}$, the 3 -ion scheme reaches its highest absorption efficiencies for such values. Since it relies on $N=1$ cyclotron heating, it also remains efficient at low temperatures. In view of the large number of gas combinations under which it can operate, it seems an ideal tool to be of assistance in the early testing phase of the machine. When relying on this heating technique during the activated phase during which $D$ and $T$ will necessarily be present in nonnegligible quantities, one should realise that fusion born $\alpha$ particles - which absorb power well away from the cold plasma ${ }^{4} \mathrm{He}$ cyclotron layer - will partly spoil the high potential of this scheme, the more since the cold $\alpha$ particle cyclotron layer lies on the low field side of the cyclotron layer of any of the candidate 3rd ion species. It would, nevertheless, be useful to study if the parasitic $\alpha$ particle RF heating can be of benefit (e.g. to indirectly heat the electrons by Coulomb collisions) or - oppositely - could be avoided. In view of the particles' high energy their Doppler shift is large so that their density profile will ultimately determine how successfully they can absorb power.

\section{NBI-ICRH synergy}

Using neutral beam injection (NBI) and ion cyclotron heating simultaneously allows performance beyond that achieved by summing the impact of using either of them. The reason for this synergistic effect lies in the fact that Coulomb collisions unavoidably slow down on charged particles. Since NBI brings particles into the plasma that have a high energy already - and hence are less collisional than the bulk population - RF waves of a given power density level can increase the energy of beam particles more than they can for thermal ones.

The benefits of simultaneously using RF and NBI heating have experimentally been known for a long time (see e.g. [24]). More recently, Krasilnikov demonstrated that the $D-D$ fusion yield can be boosted when using a $D$ beam in conjunction with RF heating heating the $D$ ions [25]. The improved performance was interpreted to be the result of increasing the amount of fast $D$ beam ions: Under the influence of Coulomb collisions the beam particles slow down and form a Maxwellian subpopulation. The constant influx of new beam source particles also creates a subpopulation with energies close to that of their birth energy. The relative fraction of these 2 populations depends on the local loss time: the longer the local loss time, the more dominant the thermalised subpopulation. RF heating allows to depopulate the thermal region and bring a fraction of the particles to higher energies, increasing the $D-D$ reaction rate. Lerche showed that "filling the gap" between the thermal region and the beam source region was consistent with the experimental evidence, both for what concerns the power deposition profile as the presence of a high energy population (yielding an increased $D-D$ neutron rate). This scheme works best when relying on fundamental cyclotron heating as this type of heating directly acts on the thermal subpopulation. Since the $D-D$ reaction rate monotonically climbs with energy, applying more power yields a higher rate. The scheme Krasilnikov relied on can be further optimised by making use of the 3-ion scheme reasoning: in a mixture of $D$ and $H$, the beam ions can be considered as a "third" species and their acceleration can be increased by properly 
positioning the beam's Doppler-shifted cyclotron layer [26].

The situation is different for the $D-T$ reaction: here the reactivity has a clear maximum. This can be exploited to do burn control, optimising the fusion reaction rate. Figure 13 shows the local density of a $D$ beam distribution RF heated at its fundamental cyclotron frequency. Three cases are plotted: (i) absence of RF power, (ii) $P_{R F}=0.2 \mathrm{MW} / \mathrm{m}^{3}$ and (iii) $P_{R F}=0.4 \mathrm{MW} / \mathrm{m}^{3}$. It can be seen how the contribution of the thermal region to the local density is gradually depleted while also a high energy tail is built (the plot has been clipped at $1.4 \mathrm{MeV}$ but the distribution has been computed with a velocity grid that goes well beyond the first zero of the RF diffusion coefficient at which it has - both theoretically and experimentally - been demonstrated that the distribution density decreases over several orders of magnitude. The fusion rate is expressed in terms of the relative velocity of the fusing particles so depending on which of the fuel ion species is heated, the optimal energy for maximising the fusion reactivity differs. For non-heated tritons, the ideal $D$ energy is just over $100 \mathrm{keV}$ while for nonheated deuterons the $T$ temperature is of the order of $160 \mathrm{keV}$. In practice the high density ensures both ion species are heated. For the adopted (core) parameters adopted for the computations in this paper, it can be shown that a modest amount of RF power allows to maximise the number of $D$ beam ions to have energies in the required range: In absence of RF heating, $8 \%$ of the particles have energies between 100 and $200 \mathrm{keV}$. This number is maximised at $P_{R F}=0.1 M W / m^{3}$ and is reduced for higher RF power densities. At $P_{R F}=0.4 M W / m^{3}$ the number of $D$ beam ions in the $[100-200] \mathrm{keV}$ range has dropped under the level in absence of the RF power.

The scheme described above is designed for fundamental cyclotron heating and makes use of $D$ beams. $\mathrm{N}=1 \mathrm{D}$ heating is - however - not compatible with the ITER-like frequency range and hence the question arises if this scheme can be used when $\omega \approx 2 \Omega_{T}$, exploiting $T$ beams. It was illustrated earlier that second harmonic heating gives rise to high energy tails while affecting the thermal population only moderately, suggesting that this technique is less ideally suited for increasing the $\alpha$ particle birth rate; on the other hand it may be useful when aiming at indirect core electron heating. Injecting a bit of ${ }^{3} \mathrm{He}$ can help channeling some power to the ions via collisional relaxation of the ${ }^{3} \mathrm{He}$ tail.

\section{Current drive}

Previous work (see $[27,28]$ ) has abundantly demonstrated that none of the heating systems foreseen for DEMO allows to drive current in a sufficiently abundant way to make DEMO a steady state machine: none of the available heating systems - and neglecting the bootstrap current dominantly created at the edge in H-mode plasmas - can generate more than a few tens of $k A$ for each $M W$ coupled to the plasma. As mentioned at the outset, the focus for present-day studies is therefore on the pulsed version of DEMO, known as "DEMO-1". In particular for ICRH, it was shown in [29] that the most optimal current drive efficiency one may hope for in the high density and temperature phase of the DEMO shot is about $70 \mathrm{kA} / \mathrm{MW}$; this necessitated avoiding parasitic alpha particle heating by launching waves in between $\alpha$ cyclotron layers (top launch), an option currently excluded [30]. Once one realises that even with this optimistic estimate, it would require $125 \mathrm{MW}$ of installed ICRH power to drive even only half of the plasma current non-inductively while only 50MW of ICRH power would 


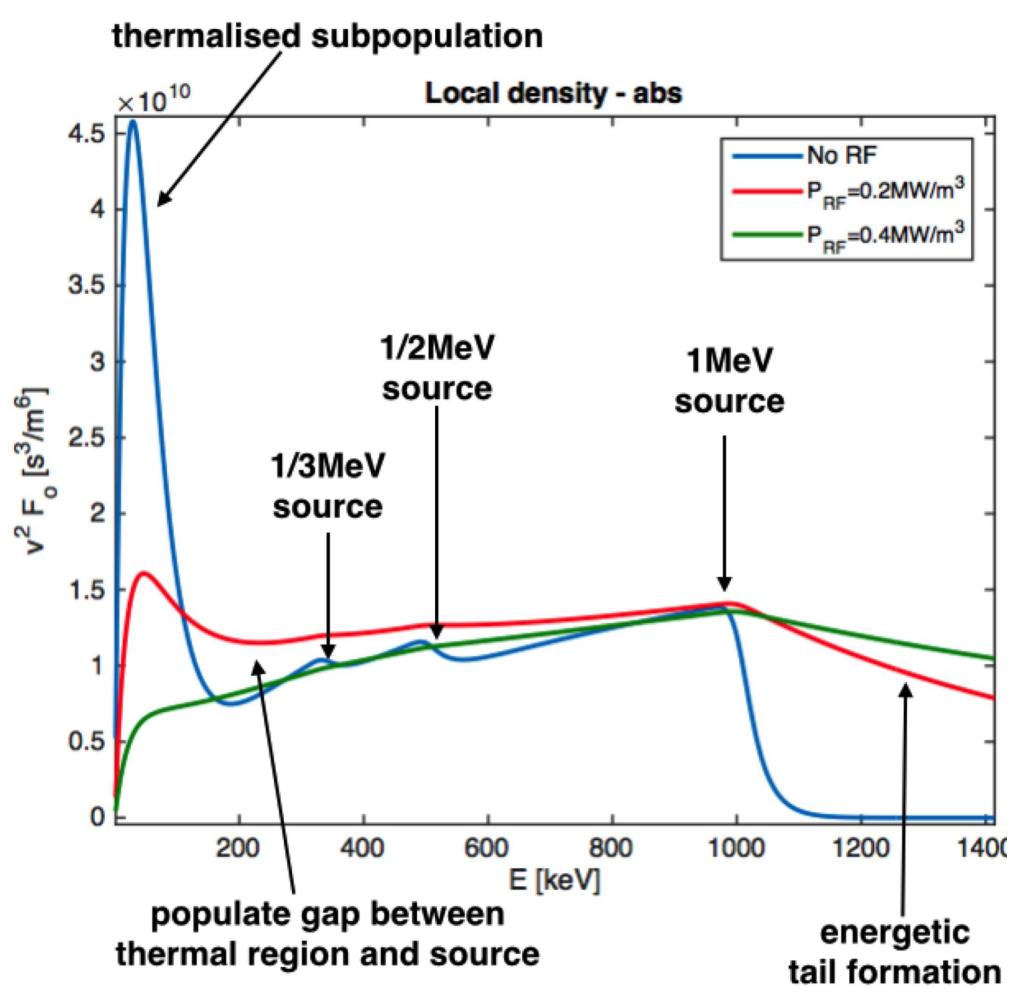

Figure 13: Local density $F_{o} v^{2}$ of a $\mathrm{D}$ beam population heated by RF heating at its fundamental cyclotron layer.

potentially be installed (hoping the rest of the current could be harvested from the bootstrap effect) it is clear that dedicated efforts should be devoted to finding alternative current drive schemes that might have a higher efficiency. Fisch [31] proposed a scheme that could be a possible candidate. It relies on purposely bringing down the plasma density and temperature - and thereby temporarily also the fusion yield - to efficiently drive current and allowing to recharge the tokamak's transformer. The current would be created in short time intervals, making use of high $\mathrm{Z}$ ions (also used to create a radiative belt close to the edge and meant to reduce the high heat flux falling on the divertor) to increase the resistivity and thereby speed up the current penetration. After that, the high density / high temperature regimes is re-installed, which allows the induced current to survive for a time much longer than the time during which it was created. Fisch's procedure makes the machine intrinsically pulsed but would - if proven viable from a (transport \& MHD) stability point of view - require significantly less installed power and might open a road towards long duration pulses on DEMO.

Exploring new current drive schemes is outside the intended scope of the present paper but relying on the expressions Ehst proposed [32], the potential of the philosophy proposed by Fisch can be illustrated. Fig. 14 depicts the driven current as a function of temperature (left) and density (right) for the flat top, full field, activated phase when driving the RF waves at a frequency of $f=65 \mathrm{MHz}$. For that frequency, the tritons absorb slightly on the high field side and the wave is dominantly absorbed by the electrons. Ehst showed that the current driven is crudely proportional to the 
temperature (aside from a further - more complicated - dependence via the electron thermal velocity) and inversely proportional to the density. This behaviour can be recognised clearly in the right subfigure of Fig. 14. In both subfigures it can be seen that the current driven in the high performance phase of the discharge is modest to very modest (of the order of 20 to $30 \mathrm{kA} / M W$ ). This is the result of the fact that the density profile is fairly flat with high core and fairly high pedestal density while the core temperature is high in this phase ("H-mode") phase. Fisch's proposal is to steer away from this regime from time to time to allow the tokamak transformer to recharge. The plots indeed show that operating at low density allows to increase the driven current significantly: at very low densities current drive efficiencies of the order of $200 \mathrm{kA} / \mathrm{MW}$ can be reached. Whether this regime is benign needs to be assessed.

Although further examination of this regime is worthwhile, it seems necessary to explore still other roads: (i) When looking into the details of the underlying heating, it can be seen that the current drive efficiency is high because the single pass absorption is low $(10-20 \%)$ so that the wave power is sloshing over the plasma many times before being absorbed. Hence it is expected that the weak main plasma absorption enters into competition with parasitic edge absorption mechanisms (collisional absorption, non-linear effects, sheath effects), the effect of which is poorly known from the theory point of view but ample experimental evidence exists to suggest this damping can easily be on par with core damping when the latter is weak. (ii) Operating at low density allows the formation of high energy tails held responsible for MHD activity. Plasma instabilities are abundantly studied but ill controlled. As will be discussed on the section of plasma landing, oscillating between high performance and low performance regimes may prove to be a delicate exercise. (iii) Also from the machine reliability point of view identifying and testing methods that can drive sufficient current to aim for steady state rather than pulsed operation is a better way forward.
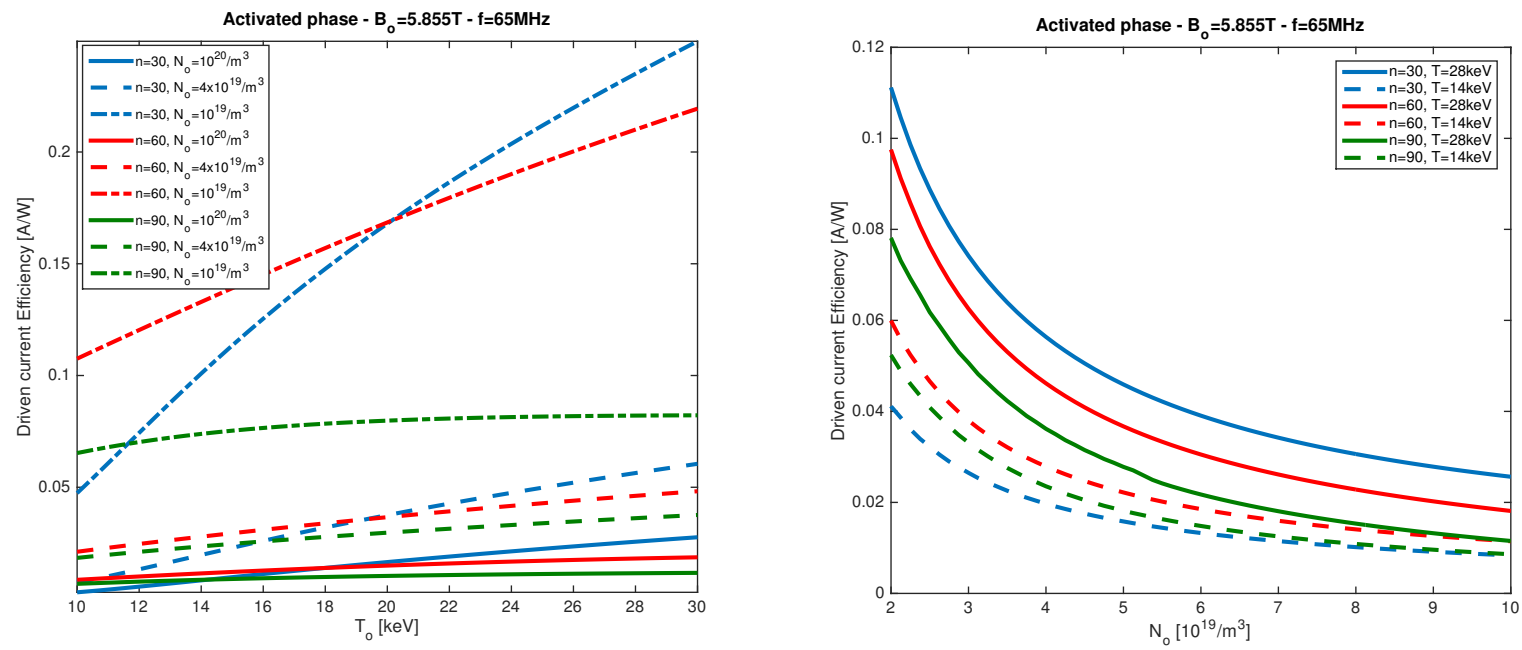

Figure 14: Current drive efficiency temperature (left) and density (right) scan for $f=65 \mathrm{MHz}$ in the flat top, full field, activated DEMO phase (no ${ }^{3} \mathrm{He}$ present)).

One option that can be looked into is minority current drive (see e.g. [33] and the references therein). Minority current drive relies on the fact that RF heating of 
minorities creates high energy tails and have a finite parallel momentum proportional to the difference between the driver frequency and the local cyclotron frequency such that it changes sign at the cold cyclotron resonance. For that reason, it is usually adopted to locally flatten or steepen the current density profile. Because the electric field and hence the absorbed power is asymmetrical w.r.t. the layer where the driven current flips sign, the integrated effect is finite, though, so that it contributes to the net plasma current. Its strength is also proportional to the energy of the minority distribution, potentially making it a potent scheme for populations of fast particles. The current is a consequence of the reduced collisionality at high energy, creating a net velocity difference between the heated and non-heated species. Bhatnagar relies on the simplified expressions of the fast ion distribution proposed for fundamental cyclotron heating $(N=1)$ by Stix [11]. It was shown in [34] that the Stix formalism can be extended to higher harmonics upon generalising the coefficients adopted; the similarities as well as the differences between distributions heated at $N=1$ and $N>1$ have been discussed earlier in this paper and in the Appendix on alpha particles. The philosophy proposed by Bhatnagar can be upgraded in a similar way and then yields currents that are asymmetrical w.r.t. to the cyclotron harmonic resonance position $\omega=N \Omega$. For the flat top phase of DEMO's shots and in the proposed frequency range, two types readily come to mind: ${ }^{3} \mathrm{He}$ minority and $\mathrm{T}$ beam particles. Alternatively at the price of going outside the currently preferred frequency window - other options are $D$ beam or even $\alpha$ particle current drive. Both of the first mentioned choices have drawbacks: In view of its price, ${ }^{3} \mathrm{He}$ is only intended to be used very early (ramp-up) and very late (landing) in the discharge. A similar drawback holds for the $T$ beam: in the ignited phase the plasma is heated by $\alpha$ particles and it is unlikely that $T$ will be injected via a beam launcher. Beam particles typically already have a finite parallel momentum while $\alpha$ particles are born with an isotropic distribution. $\mathrm{RF}$ heating distorts the distribution, making it more anisotropical. Preliminary explorations (not shown since Bhatnagar's expressions were exploited, which were developed for $N=1$ heating) suggest that this type of current drive allows driving currents of the same order of magnitude as what more classical electron current drive achieves but has the advantage that it can be operated at high density.

The final choice of the most relevant frequency interval will largely depend on the role ICRH can play. Return of experience - not in the least from the ITER machine, the activated phase of which is foreseen to start in 2036 - will determine the choice ultimately made. At this stage it is fair to say that ICRH can provide good heating but that the current drive potential is insufficient to guarantee steady state operation.

\section{The traveling wave antenna (TWA)}

The focus of the present paper is on the core plasma-wave interaction of $\mathrm{RF}$ waves launched into DEMO. Two ICRH antenna concepts are currently being considered for this machine: a "classical" one, largely building on the return of experience from standardly exploited RF antennas (ITER in particular), and a newer concept that is particularly suited for large size machines: the traveling wave antenna. Although the detailed description of this type of launcher is outside the scope of the present paper (the interested reader can find more information in dedicated papers by Messiaen and Ragona $[17,35])$, a brief sketch of its key characteristics as well as a discussion of how 
it performs is given in this section.

The fact that only the TWA is presented here in some detail should not be interpreted as a dismissal of the alternative, classical design. Ample information is available on the classical antenna type, both for what concerns present-day experimental evidence of its performance in a multitude of machines (see e.g. [36, 37]) as for the design of future machines such as ITER (see e.g. [38] and the references therein). On the other hand, information on the traveling wave antenna is significantly more scant. Little experimental evidence is available about the ability of TWA to mitigate plasma-wall interaction in metal-wall machines. High frequency ('helicon') TWAs were tested in JFT-2M [39] but they operate at high ion cyclotron harmonics and aim at imparting energy and momentum to the electrons rather than ions. A TWA test in the ion cyclotron domain is being prepared in WEST [44].

The computations discussed in the previous sections were presented making abstraction of the antenna type, treating the driver frequency and the parallel wave number as independent variables. For the TWA, the driver frequency and the location of the peak in the $k_{/ /}$spectrum are linked through the array's dispersion relation.

\subsection{Brief description of the concept}

DEMO being a large machine, the width of the density region through which the fast magnetosonic wave has to tunnel prior to becoming a propagative wave able to carry power to the plasma centre is large $(20-25 \mathrm{~cm})$. This has the direct consequence that the electric field amplitude is significant in the edge: A simple fast-wave-only back-ofthe-envelope computation shows that the power coupled to the plasma $P_{\text {coupled }}$ by a fast wave antenna and the poloidal electric field component at the antenna $E_{\text {pol,ant }}$ are related by

$$
P_{\text {coupled }}=\Delta L_{\text {tor }} \Delta L_{\text {pol }} \frac{k_{\perp}}{2 \omega \mu_{o}} \exp \left[-2 \alpha k_{/ /} \Delta R\right]\left|E_{\text {pol,ant }}\right|^{2},
$$

in which $\Delta L_{t o r}$ and $\Delta L_{\text {pol }}$ are the toroidal and poloidal extent of the antenna box, $k_{\perp}$ is the fast wave dispersion equation root at the top of the pedestal, $\omega=2 \pi f$ where $f$ is the driver frequency, $k_{/ /}$is the dominant component of the antenna spectrum, $\Delta R$ is the antenna-plasma distance and $\alpha$ is typically of order 1 . Launching $1 M W$ of power across a $20 \mathrm{~cm}$ gap using an antenna with a surface of $1 \mathrm{~m}^{2}$ and adopting parameters inspired on those of the JET tokamak $\left(k_{/ /}=6 / m, k_{\perp}=30 / m, f=51 M H z\right)$ yields a typical field strength of $E_{y, a n t}=17 \mathrm{kV} / \mathrm{m}$.

As these large fields can accelerate particles, either directly via non-resonant waveparticle interaction or indirectly via DC effects arising as a net effect of the presence of the RF fields, the presence of waves in the edge is a concern; see also the subsection on impurity chasing. Two mechanisms can readily be identified:

- In presence of a plasma, metallic structures charge negatively because of the higher mobility of electrons w.r.t. ions. This sets up sheaths, tiny regions with widths of the order of a few Debye lengths in which charge neutrality is not satisfied. Ions are attracted to the sheath while electrons are chased out of it. Radio frequency waves give rise to wave induced rectified sheaths; the amplitude of the associated rectified sheath potential scales with the RF field amplitude. At either end of magnetic field lines connecting metallic objects and passing in front 
of powered antennas, ions can be accelerated, yielding sputtering. Although the energy source driving this phenomenon is well localised - the antenna region - the effect is not. Experimental evidence has been found of plasma-wall interaction well away from the launcher (see e.g. [41]).

- The so-called co-axial modes, for which $|k|<k_{o}$, constitute a basic source of energy staying in the edge rather than penetrating the main plasma. If excited, these propagative modes carry a fraction of the wave power in the very low density region in between the main plasma and the metallic vessel wall, potentially allowing for edge particle acceleration all around the machine. Ideally, antennas should be designed to minimise the power carried by these modes. Work is ongoing to minimise this effect.

Opposite to what is the case for more conventional launchers, the straps of a traveling wave antenna are not isolated from each other by septa. Messiaen demonstrated that these septa constitute a major difference: Comparing the traveling wave antenna with an equivalent ITER-type antenna (having the same surface but including the septa and stacking the straps poloidally and toroidally) in front of a pessimistic "ITER low" density profile, he found that about 3 times more power could be launched by the traveling wave type antenna [42], allowing to reduce the near-antenna electric field magnitude and strap voltage for a prescribed power. Rather than avoiding cross-talk between the various straps, this type of antenna relies on it: Instead of feeding all the straps, only one needs to be actively fed, currents being induced on the neighbouring straps to make sure all straps contribute to the radiation of power into the plasma. The power not coupled to the plasma is evacuated via another strap (see Figure 15 discussed further). Rather than being a challenge, the large antenna-plasma distance is now an asset allowing better cross-talk between the straps and hence a more homogeneous response along the launcher. Whereas the natural response is symmetrical (fields at the left and right neighbours of the actively fed strap being affected in the same way), the system is designed to ensure the power flow becomes directional. Power leaving the last strap is re-fed into the system making use of a resonant ring configuration. The set of straps forms the equivalent of a linear structure in which characteristic modes can propagate in both directions but where the proper impedance - known as the iterative impedance of the ring configuration - is imposed at the system ends to avoid reflections, hereby creating the desired directivity giving rise to the name (traveling wave antenna) of this type of launcher. Figure 15 provides a qualitative sketch of the system. The left figure depicts 3 poloidally stacked TWA largely in front of an equatorial port but partially embedded in blanket modules.

The antenna straps are depicted in the top of the middle figure. Power enters the launcher (purple arrow) but a finite amount leaves it (blue arrow) and is recirculated. When the system is not optimally tuned, some power can flow to the load (green arrow). The transmission line feeding the antenna is connected to the generator (red dot) via an adjustable coupler consisting of 2 hybrid couplers and a line stretcher, allowing to ensure the power sent to the power dump is zero by properly adding or subtracting electric length to one of the lines connecting the hybrid couplers. The difference between the incoming and outgoing power is the sum of the power actually coupled to the plasma and the ohmic losses in the metallic current-carrying structures composing the system (transmission lines, ...). This system does not require the plasma to absorb power (in absence of damping the power - to the exception of the ohmic power 
lost in the metals - is recirculated i.e. entirely leaves the system at the last strap of the antenna, equally connected to the generator and a plasma load via the coupler). As a consequence, the TWA is automatically ELM resilient: the presence of the plasma is a mere perturbation of the system. As depicted in the right subplot of Fig. 15, the level of power coming back to the generator as well as the power ending up in the dump can be kept very small over a bandwidth of about $10 \mathrm{MHz}$. This width is comparable to that of the 'classical' antenna proposed for ITER where optimal operation is guaranteed in a $15 \mathrm{MHz}$ window [38]. At the price of reduced power launched also frequencies slightly outside that band are accessible.
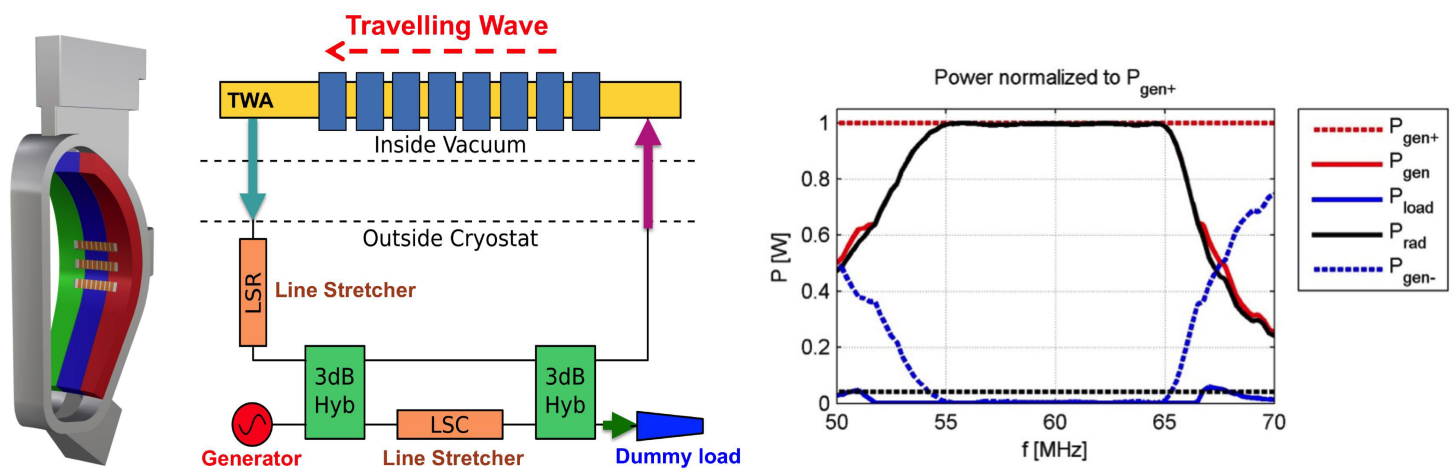

Figure 15: 3D view of the antenna integration in the DEMO blanket modules reproduced from [40] (left), qualitative sketch of the traveling wave antenna system (middle) and corresponding bandwidth (right).

Because all but one of the straps are not fed (only the first and last strap of an array are connected to the generator via a matching system; the first strap is actively fed while the last serves to evacuate power), the relative phase of the currents on the various straps cannot be imposed but follows self-consistently from the coupling of the straps: the structure acts as a band pass filter and has a characteristic dispersion relation inside the admitted bandwidth. The left and middle subplots of Fig. 16 depict a typical toroidal $(z)$ and poloidal $(y)$ spectrum of the travelling wave antenna either for 3 or 4 sections poloidally stacked on top of one another, keeping the distance between poloidal sections fixed; see the references for details. The poloidal spectrum (left subplot) has its main peak close to but not at $k_{y}=0$ and is slightly asymmetrical because of the updown asymmetry of the geometry as well as the presence of the poloidal magnetic field (causing the confining magnetic field not to point in the toroidal direction but at a small angle w.r.t. to it). For the adopted parameters the 3 -section antenna has a secondary maximum at $k_{y} \approx 5 / m$ while the 4 -section version proves to possess a somewhat more symmetrical spectrum. The toroidal spectrum is off-set, and peaks at $\left|k_{z}\right| \approx 4 / m$, both for the 3 - as the 4-section system. Note the presence of so-called "co-axial" modes $\left(|k|<k_{o}\right)$ in the middle subplot. These modes are propagative in the edge region and hence - provided their amplitude is significant - pose a potential danger for accelerating particles that can collide with the wall and give rise to sputtering. Removing the coaxial modes from the spectrum requires dedicated efforts. Antennas not relying on cross-talk between straps and where (sets of) toroidal straps are individually fed offer possibilities to shape the $k_{/ /}$-spectrum by imposing different phasings on the various 
straps, allowing to help suppress co-axial modes; the symmetrical $0 \pi \pi 0$ phasing of the ITER antenna (see Fig.23 in Ref. [38]) manages to suppress the undesired modes to large extent while other phasings cope less convincingly. Both for the classical and the traveling wave type antenna studies are ongoing to reshape the spectrum to avoid undesired parasitic effects. The plasma gyroscopy, the finite angle between the parallel and perpendicular direction, and the sensitivity of the wave spectrum reaching the main plasma on the density profile (e.g. because of the interplay between density and near-fields) make finding a robust yet flexible solution delicate. The right subplot demonstrates that the phase difference between the straps - and hence the position of the peak of the $k_{z}$ spectrum - depends on the frequency: the higher the frequency, the higher the $\left|k_{z}\right|$ value where the peak occurs. Although the position of the peak of the $k_{z}$-spectrum is unavoidably frequency-dependent for the TWA, the slope as well as the position of the axis crossing can be tuned to meet specific heating or current drive needs by adapting the design (most importantly the inter-strap distance) to meet the core physics requirements. As an illustration of the sensitivity of the launcher, the rightmost subplot gives an idea of how much the peak shifts when no dedicated effort is done to tune the capacitors optimally.

Ragona demonstrated that decreasing the number of sections for a given power increases the voltage, as expected: voltages up to $30 \mathrm{kV}$ are reached for the case of 3 while voltages up to $25 \mathrm{kV}$ are reached when using 4 sections. Some poloidal distance between the sections is needed to avoid poloidal cross-coupling, hence opting for a solution stacking sections inside a port has to be studied with care [40].
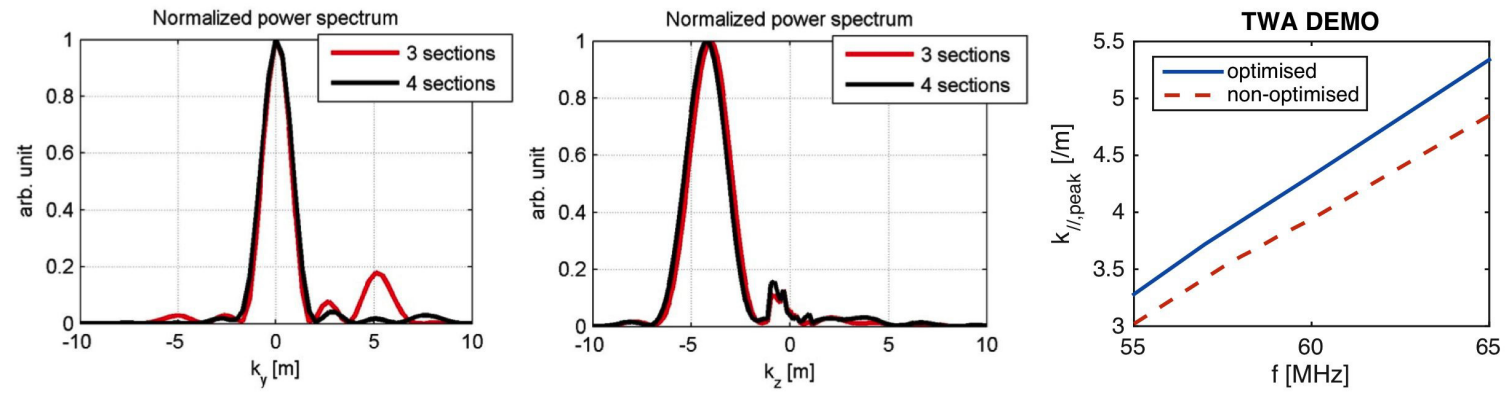

Figure 16: Antenna poloidal (left) and toroidal (middle) spectrum and position of the peak of the $k_{/ /}=k_{z}$ spectrum as function of the driver frequency (right); reproduced from [40].

It is fair to say that there is a limited theoretical understanding of the exact link between antenna characteristics and the RF sheath effects they cause; experiments provide valuable information to get a better grip on the physics involved. Aside from opting for reducing the RF power density, recent findings on the ASDEX Upgrade tokamak suggest that there is another path to mitigating the impact of the presence of large amplitude electromagnetic waves in the edge region [43] on sputtering and hot spots, possibly allowing to opt for a more "classical" antenna design for the DEMO ICRH system: increasing the distance between the antenna straps and the side-walls of the antenna box and/or reducing the currents on the straps closest to the antenna box side walls, to avoid currents being induced on the metallic parts surrounding the antenna. Present-day insights link the presence of such currents to the creation of a net $\mathrm{DC}$ voltage - closely related to the RF electric field component parallel to the static 
magnetic field - that is thought to be responsible for the unwanted ion acceleration in the edge. As it involves a modest modification of the design, efforts have already been made to incorporate this idea of extending the gaps at the end of the antenna box into the design of the TWA. On the other hand, stacking straps of a TWA-type antenna closer and closer together to reduce the volume taken away from the breeding blankets and only use the port space unavoidably yields higher power densities, taking away a key asset of the traveling wave type of antenna. A good compromise requires satisfying conflicting boundary conditions in an acceptable way.

\section{2 $N=1{ }^{3} \mathrm{He}$ and $N=2 \mathrm{~T}$ heating using the TWA}

In the general discussion reported on earlier, the heating efficiency was discussed assuming the antenna spectrum and the frequency are independent variables that can be chosen freely. When feeding all straps of a launcher independently, imposing specific relative phasings of the straps allows to impose where the location of the $k_{/ /}$peak of the antenna spectrum lies. Feeding individual strap comes with increased complexity of the total launching system, though. Relying on the mutual coupling between straps and only feeding 1 strap per TWA section takes away this degree of freedom but reduces the system's in-vessel complexity and links the location of the peak with a given frequency, as was shown in Fig.16. Limiting ourselves to the $10 \mathrm{MHz}$ bandwidth allowed for the system, the heating efficiency as a function of the frequency when accounting for the relation between $k_{/ /}$and $f$ is shown in Fig.17. The toroidal mode number varies from 39 when $f=55 \mathrm{MHz}$ to 63 when $f=65 \mathrm{MHz}$. As was already demonstrated in the general discussion, the large size of the machine makes that electron heating is always non-negligbly present. When present, the ${ }^{3} \mathrm{He}$ minority is the main absorber among the ion species but electron heating dominates that of the ions except for the lower frequencies for which the $\omega=\Omega_{3}{ }_{\mathrm{He}}=2 \Omega_{T}$ is well on the low field side. Accounting for the tail formation improves the balance in favour of the ions but is overall a weak effect.

At the usual densities, current drive is very modest $(10-20 k A / M W)$ for the present DEMO design. Exploiting Fisch's suggestion the current drive efficiency can be cranked up to more significant values: when the ion cyclotron layers of the heated ions are well on the high field side, up to $90 k A / M W$ can potentially be driven (see Fig.18). In view of the installed RF power, this is not enough to allow steady state operation but it represents an improvement that is encouraging.

Figure 19 depicts the power deposition profile for the fundamental cyclotron minority ${ }^{3} \mathrm{He}$ heating - second harmonic majority $\mathrm{T}$ heating scenario at $f=60 \mathrm{MHz}$. The top figure represents the case when the H-mode has been reached and the plasma is ignited but the minority ${ }^{3} \mathrm{He}$ has not yet had the time to diffuse out of the machine. The choice of the frequency guarantees core heating but the high density causes significant electron heating. There are 2 obvious ways to diminish the electron heating: reduce the density (second subplot from the top) or reduce the temperature (second last subplot). In the ramp-up phase both the density and temperature are low and minority heating outperforms electron heating (bottom subplot). In the $\mathrm{H}$ mode phase, all power is absorbed in a full transit over the plasma; $64 \%$ flows to the electrons, $34 \%$ to the minority and $2 \%$ to the tritons. In the L-mode phase, only $56 \%$ is absorbed per full transit, of which only $9 \%$ flows to the electrons while $44 \%$ goes to the minority; tritium absorption is of the order of $2 \%$. Keeping the density low while cranking up the 

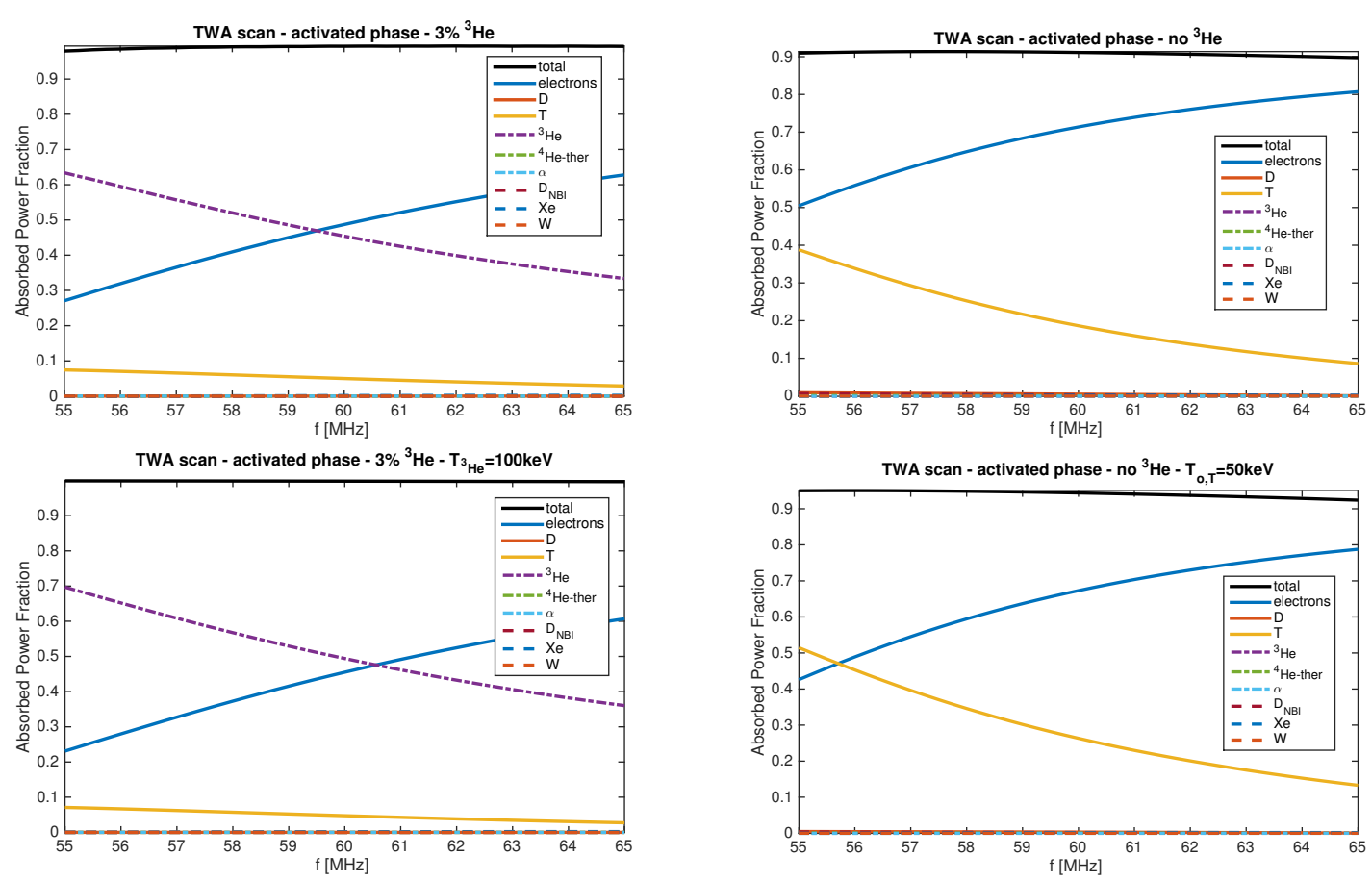

Figure 17: TWA absorption efficiency in the bandwidth and accounting for the relation between the driver frequency and the antenna spectrum for the high performance plasma phase: with (top left) or without (top right) $5 \%$ of ${ }^{3} \mathrm{He}$ but not accounting for the $\mathrm{RF}$ induced tail, and like wise when assuming $T_{3} \mathrm{He}=100 \mathrm{keV}$ (bottom left) or $T_{T}=50 \mathrm{keV}$ (bottom right).

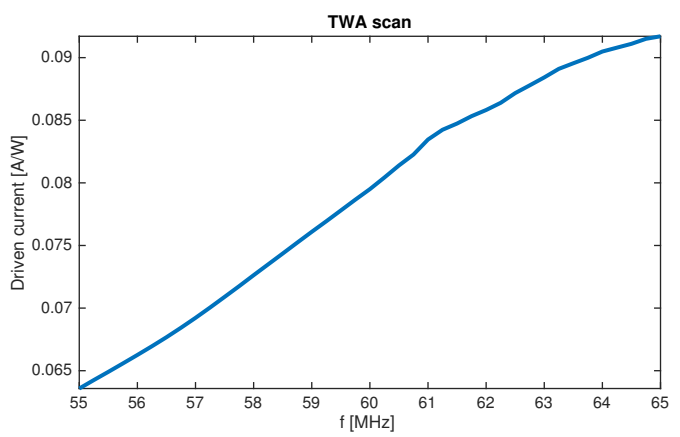

Figure 18: Current drive efficiency for the TWA.

temperature yields near-total absorption (93\%), dominantly by the minority (56\%) and partly to the electrons (36\%). Again, T heating is finite but minimal (1\%). Operating at high density when the temperature is low yields the worst efficiency: only slightly over half of the power is absorbed (56\%) in a full transit, of which $44 \%$ by the minority and $11 \%$ by the electrons. This suggests that the optimal way to reach ignition is by keeping the density modest to allow the majority to reach fusion relevant temperatures, after which the $\alpha$ particle heating gradually allows to increase the density while further increasing the temperature. 

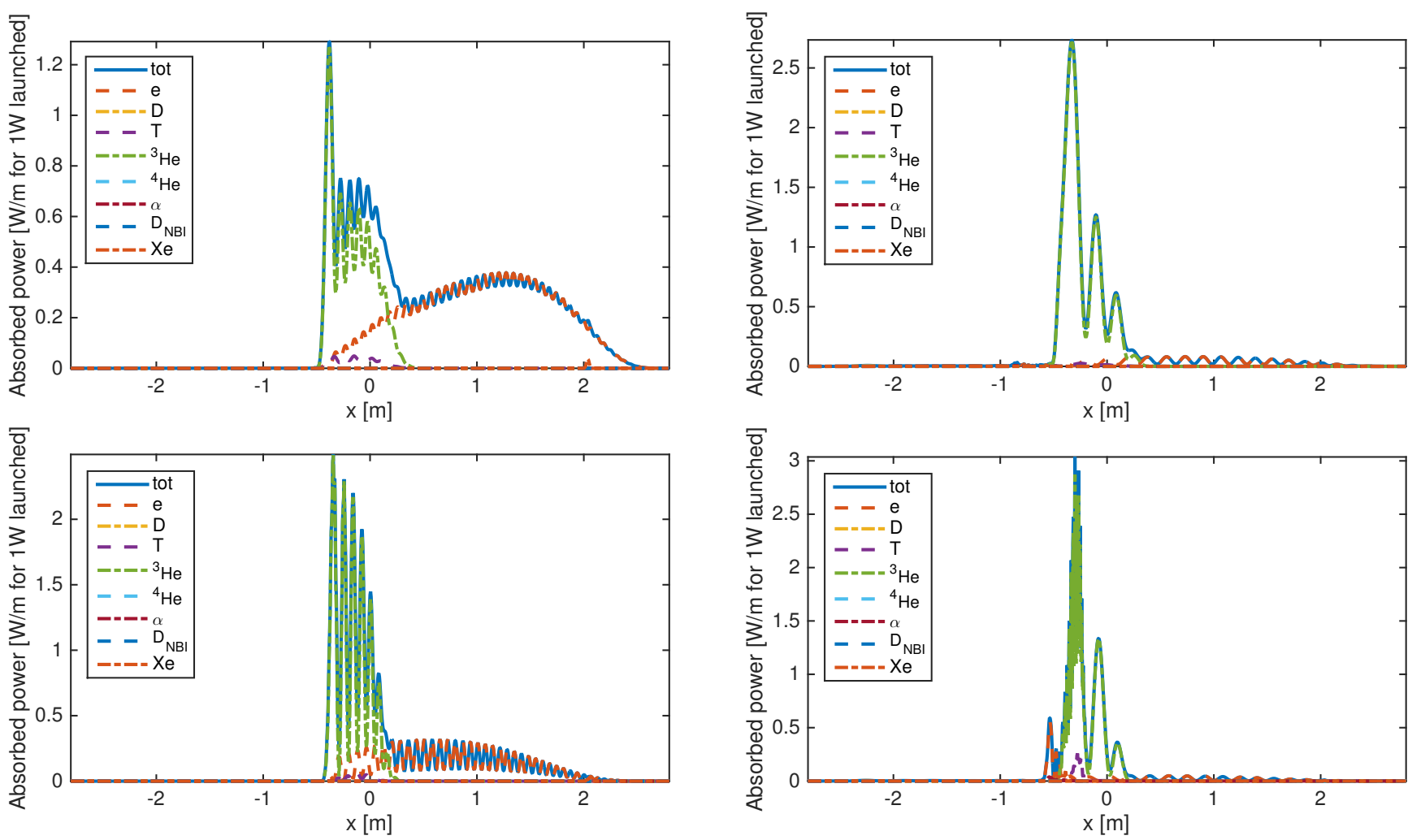

Figure 19: RF power density for the reference $D-T-{ }^{3} \mathrm{He}$ scenario for 4 relevant cases: (i) high density and temperature $\left(N_{e, o}=10^{20} / \mathrm{m}^{3}, T_{o}=28 \mathrm{keV}\right.$; top left), (ii) low density and high temperature $\left(N_{e, o}=0.2 \times 10^{20} / \mathrm{m}^{3}, T_{o}=28 \mathrm{keV}\right.$; top right), (iii) high density and low temperature $\left(N_{e, o}=10^{20} / \mathrm{m}^{3}, T_{o}=14 \mathrm{keV}\right.$; bottom left) and, finally, (iv) low density and low temperature $\left(N_{e, o}=0.2 \times 10^{20} / \mathrm{m}^{3}, T_{o}=14 \mathrm{keV}\right.$; bottom right $)$.

\section{ICRH tasks besides heating and current drive}

Efficient transfer of energy from launchers outside the last closed flux surface to the main plasma is a necessary but not a sufficient condition for ignition. The present paper illustrates the potential of radio waves to (help) bring DEMO plasmas to fusion relevant temperatures but purposely limits itself to that. It sidesteps issues such as the stabilising or destabilising effect wave induced high energy populations have on MHD, or wave induced turbulence impacting on transport. In the previous sections the benefit of using a minority fundamental cyclotron heating scheme in the ramp-up phase has been underlined. Approaching fusion-relevant temperatures, majority second harmonic heating is a good candidate for taking over the task of plasma heating. It was illustrated, e.g. in Figs.6 and 10, that guaranteeing high single pass absorption and making sure power flows to the ions - prerequisites for reaching fusion relevant ion temperatures - can be done by properly choosing the plasma parameters. This was already successfully demonstrated experimentally in the first D-T campaign in JET $[45,46]$, ensuring efficient core ion heating and producing (i) the highest steady-state fusion Q-value reached relying on $D$ minority heating, or (ii) boosting the neutron rate using ${ }^{3} \mathrm{He}$ minority in $\mathrm{T}$.

But a key difference between JET in the 90's and future fusion reactors is that sus- 
tained performance in presence of metal rather than carbon walls needs to be demonstrated. The modelling of this more delicate dynamics is not attempted here. Access to the high confinement ('H') mode is considered to be crucial: A glance at the energy confinement scaling law (see e.g. [47]) readily demonstrates that for a prescribed amount of net power passing the last closed flux surface to reach the main plasma, crossing the $\mathrm{L}(\mathrm{ow})-\mathrm{H}(\mathrm{igh})$ mode threshold yields a significant increase of the time the plasma can contain energy. Somewhat surprisingly in view of the very different dynamics associated with them, it is thought that it is immaterial which heating source is exploited, suggesting that heating methods are interchangeable. This assessment stems for scaling laws which are not making this distinction either. Aside from size, the plasma current is the main parameter influencing confinement. The power needed to cross the H-mode threshold has experimentally been crudely established to scale somewhat less than linearly with average density and toroidal magnetic field strength $\left(P_{L-H} \propto N_{a v}^{0.60} B_{t o r}^{0.57}\right.$ according to Snipes [48] and $P_{L-H} \propto N_{a v}^{0.717} B_{t o r}^{0.803}$ according to Martin [49]). More detailed analysis shows a minimum as a function of density; see e.g. the JET evidence in Fig.11 in [50], illustrating the L-H threshold crossing at low field solely relying on ICRH. This suggests two competing mechanisms, one dominating at low and one dominating at high density. Singh argues that plasma turbulence competes with radiation [51] while Fundamenski substitutes the latter for the shear Alfvén wave [52]. The insights of the impact of ICRH on MHD activity and plasma turbulence need to be deepened; see the subsections on sawtooth pacing and plasma landing. Current views on transport predict that of the order of $50 \mathrm{MW}$ of auxiliary power will allow to bring DEMO to ignition [1] while crudely half of that should suffice to enter the $\mathrm{H}$ mode regime.

\subsection{ICRF assisted plasma start-up and wall conditioning}

Production of current-less ICRH-created plasmas is studied on present-day tokamaks to check if RF assisted start-up $\left(N_{e}>10^{18} \mathrm{~m}^{-3}\right)$ and wall conditioning applications (ICWC, $N_{e}<10^{17} \mathrm{~m}^{-3}$ ) can successfully be applied in future superconducting fusion devices. Tokamak pre-ionisation assistance by ICRF fields was demonstrated in Europe (TEXTOR [53]) and China (HT-7 [54]). Separately, it is shown on JET that on-axis, peaked density profiles $\left(>10^{18} \mathrm{~m}^{-3}\right)$ can be obtained in ICRH-created plasma, both in the inverted minority $H-(D)$ scenario at $\omega=\Omega_{D}$ and the standard minority $H-(D)$ scenario at $\omega=\Omega_{H}$ (for examples, see Fig.20 in this paper and Fig.2 in reference [55]). The minority species in these experiments $(5-10 \%)$ are fuelled solely by the plasma facing components. The density is achieved at modest generator power levels below $0.6 M W$ with coupling efficiency of $60 \%$, at neutral pressure of $5 \times 10^{-5}$ mbar. The transport processes that govern these current-less plasmas are excited/driven by the curvature and grad-B drifts, which scale as $1 / R$. Hence a scaling with $R^{2}$ rather than $R^{3}$ - more typical for a volume effect - is expected. Scaling the power level from $J E T$ to $D E M O$ as $R^{2}$, this amounts to a modest $5 M W$ of power needed for initiating the plasma in a suitable heating scenario, i.e. $10 \%$ of the installed ICRH power on DEMO. Start-up is typically done relying on a minority fundamental heating scheme. When the ITER-like ICRH heating scenarios are preferred this requires the use of ${ }^{3} \mathrm{He}$ when DEMO is operated at full $B_{o}$ field and adopting the preferred frequency range of $55-65 \mathrm{MHz}$. Alternatively minority second harmonic heating of a $T$ minority could be envisaged but this scheme's efficiency at low temperature is not guaranteed. 
One could also opt for a dedicated low power antenna that can heat a $\mathrm{D}$ minority at $f \approx 45 \mathrm{MHz}$. The sufficiently dense ICRH-created plasma may be ideally suited as target plasma, allowing subsequent current ramp-up, eventually combined with further wave heating by ion or electron cyclotron waves. Advantages are a reduced magnetic flux consumption, extended gas pressure range and tolerable residual impurity content.
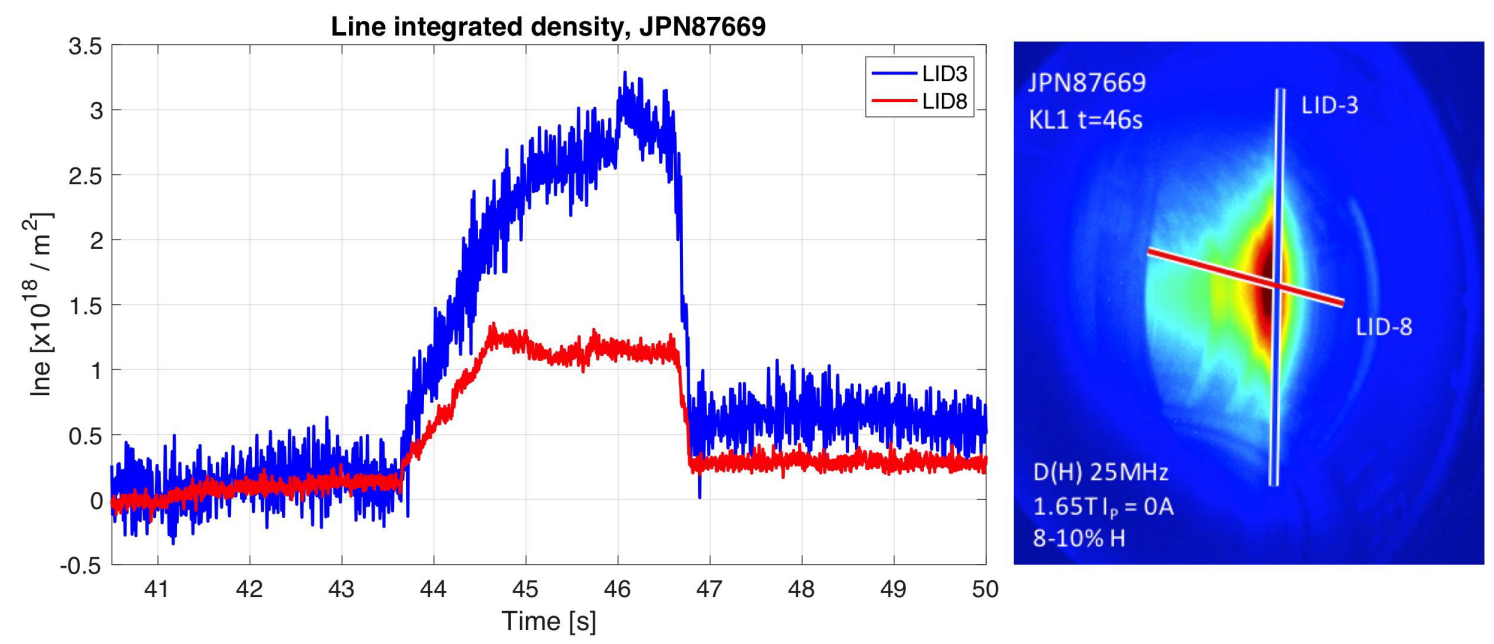

Figure 20: Line integrated densities by DCN interferometry (left) and $D_{\alpha}$ radiation image with normalised pixel intensity (right) from JET KL1 monochrome CCD camera for an ICRF discharge, shot 87669 , with indication of the vertical and radial interferometry viewing lines. The images show formation of an on-axis localised plasma radiation zone.

Wall conditioning is commonly applied in magnetic confinement fusion devices to improve plasma performance and discharge reproducibility by controlling the content of the plasma-facing surfaces. Also on DEMO, ideally operated in quasi steady state and with hot walls $\left(>400^{\circ} \mathrm{C}\right)$, wall conditioning can facilitate the initial plasma breakdown and current ramp-up. ITER relies on ICRF conditioning for providing tritium safety. ICRF plasma recovers Tritium from retention dominated areas and mitigates such a tritium inventory build-up. The same applies to DEMO, with even an additional requirement for tritium-self-sufficiency. Numerous experiments evidenced the effectiveness of ICWC, demonstrating (i) recovery of sustained ohmic breakdown and density control after disruptions (realised e.g. on Tore Supra [56]) and after severe impurity exposure (evidenced in TEXTOR [57]), (ii) full change-over of the isotopic ratio of the JET ITER-like wall [58], (iii) full depletion of the Tungsten ASDEX-Upgrade wall from deuterium by He-ICWC [59] (iv) higher density, temperature and purity of the main plasma in the U-3M stellarator after ICWC performance [60].

Both ICWC discharges and ICRF pre-ionisation plasmas for start-up assistance start with the process of ICRF plasma breakdown. This is the ionization avalanche initiated by electrons accelerated in the $E_{/ /}(z, t)$ parallel electric field in the vicinity of ICRF antenna. An analytical method was developed in order to pre-assess the optimal parameters for discharge initiation by ICRF antennas [61]. The method complements the self-consistent but CPU intensive simulations by the Particle-in-Cell Monte Carlo Collision code RFdinity1D [62]. It builds on the ponderomotive description of electron acceleration in the vacuum parallel electric field $E_{/ /}(z, t)$. Good agreement being found 
between the simulations and the experimental evidence suggest the key dynamics has been understood.

\subsection{Impurity chasing}

A key challenge for DEMO remains the fact that one simultaneously needs to keep the heat load on the divertor manageable while ensuring the plasma core is not contaminated by high $\mathrm{Z}$ material. ITER opts for $\mathrm{Be}$ as the first wall and $\mathrm{W}$ as the divertor surface material. Tungsten has a very high melting point $\left(3422^{\circ} C\right)$ but - while Beryllium has a charge of only 4 - that of tungsten is 74 . When extrapolated to the DEMO design, Be layers will not be suitable due to radiation damage but $\mathrm{W}$ is likely still to be exploited. Whereas tungsten is purposely chosen for the divertor to withstand the high heat loads, penetration of minute amounts of tungsten into the main plasma enhances the radiation and hence e.g. the risk for disruptions triggered by a thermal quench [63]. Nowadays, strike point sweeping is routinely used at JET to spread the heat load over a bigger area of the divertor. More elementary still - as the presence of high $\mathrm{Z}$ material in the core degrades the temperature that can be reached - it has a direct impact on fusion yield. Methods are being investigated to remove impurity populations so that core radiation is modest. Already in the 90's the role of ICRH was realised in that respect: In TEXTOR - a device dedicated to plasma-wall interaction - it was demonstrated that tungsten could be chased successfully out of the plasma by applying ICRH at a sufficient power level [64]. The dynamics of the then observed effects are instructive: Shortly after switching on the radio frequency (RF) generators, the tungsten level increased due to enhanced interaction with the vessel wall but in a quickly reached stationary regime the core tungsten concentration subsequently decreased to a negligible level provided enough ICRH power was available. More recently, the same has been observed in JET when using hydrogen minority heating in deuterium majority plasmas: Provided sufficiently high core temperatures are achieved, the core concentration of tungsten is decreased $[65,66]$. Also in ASDEX-U, the removal of high $\mathrm{Z}$ material relying on ICRH was proven [67].

How high $\mathrm{Z}$ chase-out works exactly is still being debated. Neoclassical transport affected by poloidal asymmetries and turbulent transport is currently thought to be the dominant mechanism responsible for high $\mathrm{Z}$ accumulation in the core of the plasma [19]. The underlying mechanism for the former is explained by Bilato [68], who links a poloidally (high - low field side) asymmetrical heated minority profile to the impurity transport. Depending on the bulk plasma profiles, neoclassical temperature screening can - however - prevent accumulation, an effect that can further be enhanced by externally heated species. Valisa argues that the sign of the neoclassical pinch can be reversed when the temperature is peaked in the center [18]. Angioni in turn argues that the neoclassical inward pinch can be counteracted by turbulent transport due to the capacity of the central RF heating to increase the latter at the expense of the neoclassical effects [69], the net transport being the combination of both types of transport. Whichever of these effects is the cause of the observed chase-out, it is fair to state that $\mathrm{ICRH}$ can also play a role in avoiding accumulation of high $\mathrm{Z}$ populations in the core of DEMO.

Although it is not yet clear which physics aspect is the key actor to impurity chasing, all current-day high $Z$ removal schemes involve core fast particle populations. Hence one may pose the question if the minority population of fusion-born alphas themselves 
could contribute to impurity chasing. Alphas were already mentioned in the context of minority current drive but it would require an ICRH scheme aiming at heating the fuel ion $\mathrm{D}$ majority - and hence equally $D$-like populations such as alphas - to ensure wave power flows to the fusion products to break the isotropy of the $\alpha$ distribution to ensure a net current. Such type of parasitic wave heating is not commonly considered as very desirable. But for impurity chasing, it is possible that the high energy itself - and not the distribution's anisotropy - is of dominant importance. It would be interesting to address this question relying on sufficiently sophisticated codes, and to monitor this aspect in the upcoming JET D-T campaign (see e.g. [70]).

The need for finding efficient ways to chase impurities from the main plasma could likely be avoided provided the source of impurities can be eliminated i.e. provided the sputtering thought to lie at the origin of the impurity influx can be reduced to a tolerable level. Due to the presence of large electrostatic or electromagnetic electric fields in the edge, charged particles can be accelerated and bombard the wall. RF electric fields close to the antenna are evident candidates for causing such net acceleration. Ions on orbits intersecting the metal vessel wall can be further accelerated in the sheaths (formed close to metal objects as a result of the difference in electron and ion mobility resulting in metallic objects charging negatively and subsequently attracting ions while repelling electrons). Understanding the dynamics of this process is challenging. The presence of large electric fields e.g. gives rise to RF sheaths, the voltage drop across which grows with the RF electric field magnitude and exceeds that of the usual sheath. While the sheath dynamics require zooming in to the Debye length scale, studying the fate of particles accelerated close to the antenna but on orbits that only intersect metallic objects well away from the antenna requires models that are able to capture macroscopic evolution. Aside from the numerical difficulties of describing the edge in view of these vastly different length scales there is a more basic problem: the assumptions underlying models used are often violated when applying them for understanding edge phenomena. For example, fluid-type equations implicitly assume particle populations have shifted Gaussians as distribution functions, having had the time to collisionally redistribute so that their motion can be characterised well by a single fluid velocity. But populations accelerated in the edge and on orbits intersecting metallic objects lack the time to thermalise, necessitating a kinetic description to grasp the details of the wave-particle interaction dynamics. Through a combined use of fluid, kinetic and Monte-Carlo methods and through experimental evidence, progress is gradually made to get a better grip on the wave-particle interaction in the edge, though. One example is the redesigning of antennas to avoid currents flowing on metallic objects surrounding the antenna box mouth. The philosophy is based on the assumption that the slow wave's parallel electric field is the main driver of wave driven particle acceleration in the edge and that image currents on the sides of the antenna box - giving rise to the undesired field components - can largely be avoided by enlarging the distance between the antenna straps and the box wall. Adopting a dedicated antenna after having studied the reduction of $E_{/ /}$through modelling, it has been demonstrated experimentally that the impurity level can be reduced significantly $[43,71]$. This straightforward recipe has been adopted in the traveling wave antenna design but awaits experimentally being tested for this type of antenna. To be able to numerically cope with the very different scale lengths, work is being done to upgrade the metal boundary condition used in wave equation solvers to a boundary condition 
that accounts for the presence of the sheath. D'Ippolito and Myra have pioneered this approach $[72,73,74]$, which is still being upgraded. A key difficulty is that the simultaneous presence of fast and slow waves in a magnetised plasma in which the density is changing significantly over Debye scale distances and in which charge neutrality is not satisfied makes it hard to come up with a sufficiently general and yet suitably simple boundary condition, as is e.g. illustrated in Ref. [75] in which the impact of effects such as the orientation of the magnetic field and oblique incidence have been studied numerically. A supplementary difficulty is that the density is macroscopically changed by the presence of the RF electric field. The creation of convective cells in front of RF antennas was experimentally demonstrated by Van Compernolle [76]. The observed convection pattern - resulting in density inhomogeneities affecting the coupling of the waves to the plasma - can qualitatively be understood as due to ponderomotive effects (see e.g. [77, 78] and the references therein) but has not yet been modelled full self-consistently. Finally, wheres the Lorentz force due to the confining magnetic field dominates the motion of particles in the hot plasma core, the Lorentz force contribution from the RF field is equally strong as that of the confining field, implying that the notion of magnetic confinement is lost close to $M W$ wave launching structures.

Efforts are ongoing to tackle the impurity problem at the source and to acquire a better understanding of the physics involved.

\subsection{Sawtooth pacing}

When the safety factor drops below 1 in the center of the plasma, sawteeth [79] occur: a slow rise of the temperature is followed by an abrupt crash, restoring the temperature approximately to the value it had at the start of the cycle. According to Park et al. [80] the sawtooth period scales with $T^{3 / 2}$. As a consequence heating tends to lengthen the sawteeth. Monster sawteeth are indeed a signature of efficient core heating [81]. While ELMs ensure flushing of particles across the edge, sawteeth are believed to be beneficial for helping to evacuate impurities from the core. Large sawteeth can, however, trigger more dangerous instabilities, such as the neo-classical tearing mode [82], which significantly degrade confinement and can give rise to disruption of the plasma. Too frequent sawteeth tend to mildly degrade the core confinement while too long sawteeth carry the risk for instabilities. Being able to force the sawtooth oscillation frequency is therefore thought to be useful. Park argues that the sawtooth period also scales with the inverse of the effective charge, suggesting that the impurity content and the sawtooth activity are intimately linked.

Sawtooth pacing experiments have been conducted successfully in a number of machines using ECRH [83] but also using ICRH [84]. The main requirement is ensuring power is deposited close to or inside the $q=1$ surface. Depositing power at the $\mathrm{q}=1$ surface mainly tries to deform the current density profile (i.e. is a current drive rather than a heating effect) and influence the sawteeth at their inversion radius. Sawtooth pacing by depositing power inside the $q=1$ surface aims at creating a core fast ion population. Since the slowing down of this fast population - dominantly on the electrons - is a necessary ingredient, this type of sawtooth pacing is inherently slower than pacing based on q-profile perturbations resting on local current drive. The main advantage of the latter scheme is that the exact location where the power is deposited is less critical as long as it is inside the $q=1$ surface. For example for maximizing the fusion yield, core heating is commonly preferred. Core heating is also needed for ensuring a peaked 
temperature profile, known to influence the neoclassical pinch velocity and therefore to impact on chasing of high $\mathrm{Z}$ particles from the core.

As was shown earlier, ICRH allows to decide where ions are preferentially heated by a proper choice of the driver frequency for a given magnetic field and allows to explore both options allowing pacing.

\subsection{Plasma "landing"}

Ending high power discharges in metal wall machines has proven to be challenging. The plasma energy dropping faster than the density when switching off the main heating tends to enhance the influx of high $\mathrm{Z}$ material to the core, causing increased radiation and making the temperature profile hollow - deforming the current density profile and giving rise to enhanced MHD activity - or even leading to a thermal quench of the plasma. Disruptions put high stresses on the machine and need to be avoided at all cost, hence "landing" the plasma safely after a high density, high temperature and high current phase has been a point of attention. Core RF heating has proven to allow avoiding high $\mathrm{Z}$ influx, mitigating the risk for the inverted temperature profiles. Various explanations have been offered (see the section on high $\mathrm{Z}$ chase-out) but they all have 1 ingredient in common: increased core electron temperature.

From the discussions above, it is clear that having dominant electron heating is not particularly hard in DEMO. Ensuring significant direct core electron heating is less trivial, but as second harmonic $T$ heating gives rise to high energy tails, core $T$ heating will give rise to indirect electron heating at that location. However, the efficiency of second harmonic heating dwindles when the temperature goes down. As high fast ion pressure guarantees indirect electron heating as well, relying on minority heating as is done in the start-up phase is an obvious tool to end the discharge.

JET experiments show that another crucial element is having control of the ELM frequency, ensuring outflushing at the edge $[85,86]$ while gradually stepping down the neutral beam power (the most prominent heating scheme at JET, allowing to going into deep H-mode at high current and field). De Vries argues that reducing the auxiliary power reduces the ELM frequency, causing the density to decay slower than the current, hitting the Greenwald limit and limiting the duration of the $\mathrm{H}$ mode phase and ultimately the pulse. In the upcoming D-T campaign of JET landing the plasma will be one of the issues getting attention in preparation for future machines.

\section{Reconsidering the frequency range}

The key reason why second harmonic $T$ heating is preferred over fundamental $D$ heating is that the latter unavoidably yields to RF heating of a subpopulation of the fusion born $\alpha$ particles to even higher energies than their birth energy. The discussion on the 3 -ion options as well as that of the current drive and the landing suggests that another frequency range might be considered. On top of that, it is worthwhile to mention that the $D$ minority heating scheme - with optimal $D$ concentration of the order of $10 \%$ - proved to be a promising $D-T$ heating scheme in the first JET $D-T$ campaign (see e.g. [45, 46]) when only relying on RF heating, and has the potential of being significantly improved when exploitation the RF+NBI synergy (see e.g. [87] proposed to be tested in the upcoming JET $D-T$ campaign). At this stage, the arguments in 
favour of the [55-65] $\mathrm{MHz}$ range outweigh those for lower or higher frequency ones but further modeling and - more importantly - experimental evidence may deliver return of experience suggesting keeping other options open. From the RF design point of view, there is no fundamental problem aside from the fact that the launcher design needs to be moderately reviewed.

\section{A note on the impact of transport}

A prerequisite for plasma heating is the availability of efficient heating mechanisms. The fate of RF power once it is transferred from the waves to the particles is determined by diffusion processes occurring on a much longer time scale than that accounted for in the wave and Fokker-Planck equations. The detailed study of various relevant - neoclassical and anomalous - transport mechanisms is an item of study in its own right and will not be attempted here. But a simple transport model (described in Appendix D) allows to provide an idea of the impact $50 M W$ of ICRH power can have on the DEMO plasma. Figure 21 shows the temperature modifications brought about by $50 M W$ of RF power coupled to the DEMO plasma in the ignited phase when one of the fuel ion species is directly heated while the electrons equally absorb RF power; for simplicity, it has been assumed that the alpha particle power flows to the electrons so that no dedicated transport equation is needed for this minority specie. The set of coupled transport equations is solved adopting the following parameters: We consider an analytical D-shaped equilibrium with elongation 1.65 and a triangularity 0.3333 conform with the DEMO reference design that was considered earlier; the reference density $\left(N_{o}=10^{20} / \mathrm{m}^{3}\right.$ and $\left.N_{s}=0.567 \times 10^{20} / \mathrm{m}^{3}\right)$ and temperature $\left(T_{o}=28 \mathrm{keV}\right)$ as well as the confinement time $(\tau=3.6 s)$ are also taken from the same information source. The top subfigures in Fig. 21 depict the source power density and the corresponding integrated power as a function of $\rho$ (the flux surface labeling parameter varying from 0 at the magnetic axis to $a_{p}$ at the plasma edge), with most of the power flowing to the electrons and the electron deposition profile larger than that of the ions. A modest inward pinch velocity of $0.1 \mathrm{~m} / \mathrm{s}$ was assumed. For the given density and temperature, the above transport equation system is first used to determine the reference power density source consistent with the given density and temperature profiles; as anticipated this source power is much larger than the RF power. The temperature profiles consistent with the extra $50 \mathrm{MW}$ of $\mathrm{RF}$ power are then determined solving the set of coupled transport equations. The difference $\Delta T_{j}$ between the given reference profiles $T_{j}\left(P_{\text {source }}\right)$ and the obtained ones $T_{j}\left(P_{R F}+P_{\text {source }}\right)$ is subsequently plotted in the bottom subplots of Fig.21. Peaked temperature modification profiles result. Due to the high density, the impact of the thermal equilibration is significant, causing the D fuel ions that are not directly heated to obtain a temperature similar but somewhat lower to that of the tritons and the electrons. The more peaked ion power source profile results in the triton temperature exceeding that of the deuterium and electrons. The relative importance of the diffusion and the equipartition is clearly seen when increasing $K_{\rho \rho}$, yielding a bigger temperature difference, separating species that are directly heated from species that are not. Although strictly the diffusion coefficient is inhomogeneous (different - neo-classcial and anamalous - transports mechanisms dominating in different regions and having differing dependencies on the density, temperature, ...) it has been kept constant here. Increasing the diffusion also yields wider profiles but 
smaller overall temperature differences. Realistic values for $K_{\rho \rho}$ are thought to be of the order of a few $\mathrm{m}^{2} / \mathrm{s}$ and hence one expects only a temperature difference of the order of $1 \mathrm{keV}$ resulting from $50 \mathrm{MW}$ of power in the ignited phase.

The ITER L and H mode scalings laws [88, 89] predict the DEMO energy confinement time will be of the order of $0.5-1 s$ in the ramp-up phase and will increase to $\approx 3-4 s$ in the ignited phase. The heat diffusion coefficient is equally expected to grow when the density increases; $K_{\rho \rho}$ is of order $0.2 \mathrm{~m}^{2} / \mathrm{s}$ in the ramp-up phase and of order $1.5 \mathrm{~m}^{2} / \mathrm{s}$ in the activated phase. Figure 22 gives an idea of the central temperature increase when applying an extra 50MW of power. It was assumed that $100 \mathrm{MW}$ of auxiliary heating power (likely from different heating methods) is available in the first (L-mode) phase and that the heating in the latter (H-mode) phase is ensured by $400 M W$ of $\alpha$ particle heating consistent with the $2 G W$ of fusion power produced.

For the latter, the temperatures and densities used in the wave equation study were used; for the ramp-up a core reference temperature of $8 \mathrm{keV}$ and a reference core density of $2 \times 10^{19} / \mathrm{m}^{3}$ were assumed. None of the imposed parameters were computed self-consistently; a more careful check of the transport is required.

In the ramp-up phase, the equipartition is modest due to the low density, yielding different temperature evolutions for the differently heated species. For the RF power densities taken (same as in the previous figure), the non-directly heated deuterons' central temperature maximally increases by $14 \mathrm{keV}$, while that of the heated species is raised by $18 \mathrm{keV}$ for the electrons and $24 \mathrm{keV}$ for the tritons. In the ignited H-mode phase, the core temperatures increase much less significantly for the same amount of input power, as the diffusion is stronger while the confinement time longer. Moreover, the higher density yields stronger equipartition and temperature differences that are much less pronounced, in particular compared to the reference temperature $(8 \mathrm{keV}$ in the L-mode and $28 \mathrm{keV}$ in the H-mode case).

\section{Sensitivity of the obtained results to changes of the machine parameters}

Over the years, the DEMO parameters have undergone various modifications. The present section briefly looks into the impact of such changes on the absorption efficiency, focussing on 2 design parameters that were subject to change in the past in the quest to optimise the machine: large aspect ratio $a_{p} / R_{o}$ and magnetic field strength $B_{o}$.

Figure 23 shows how the absorption efficiency changes as a function of the frequency for 3 different values of the aspect ratio during the activated phase: $0.25,0.3$ and 0.35 . The adopted toroidal mode number is $n_{\text {tor }}=30$ and all other parameters except the minor and major radius are as given at the outset of the discussion. Both the minor radius $a_{p}$ and the major radius $R_{o}$ were adjusted while keeping the volume constant. It is readily observed that the overall behaviour changes little, the various plots crudely corresponding to those discussed in Fig. 3 (except that a different $n_{\text {tor }}$ - more in line with the TWA concept - was adopted there). The interval around $60 \mathrm{MHz}$ in which both dominant electron heating and dominant ion heating are guaranteed upon a moderate frequency range compatible with $10 \mathrm{M} \mathrm{Hz}$ bandwidth slightly shifts towards lower values for increased $a_{p} / R_{o}$. One concludes that $\mathrm{RF}$ heating is fairly insensitive to modifications of the aspect ratio. 

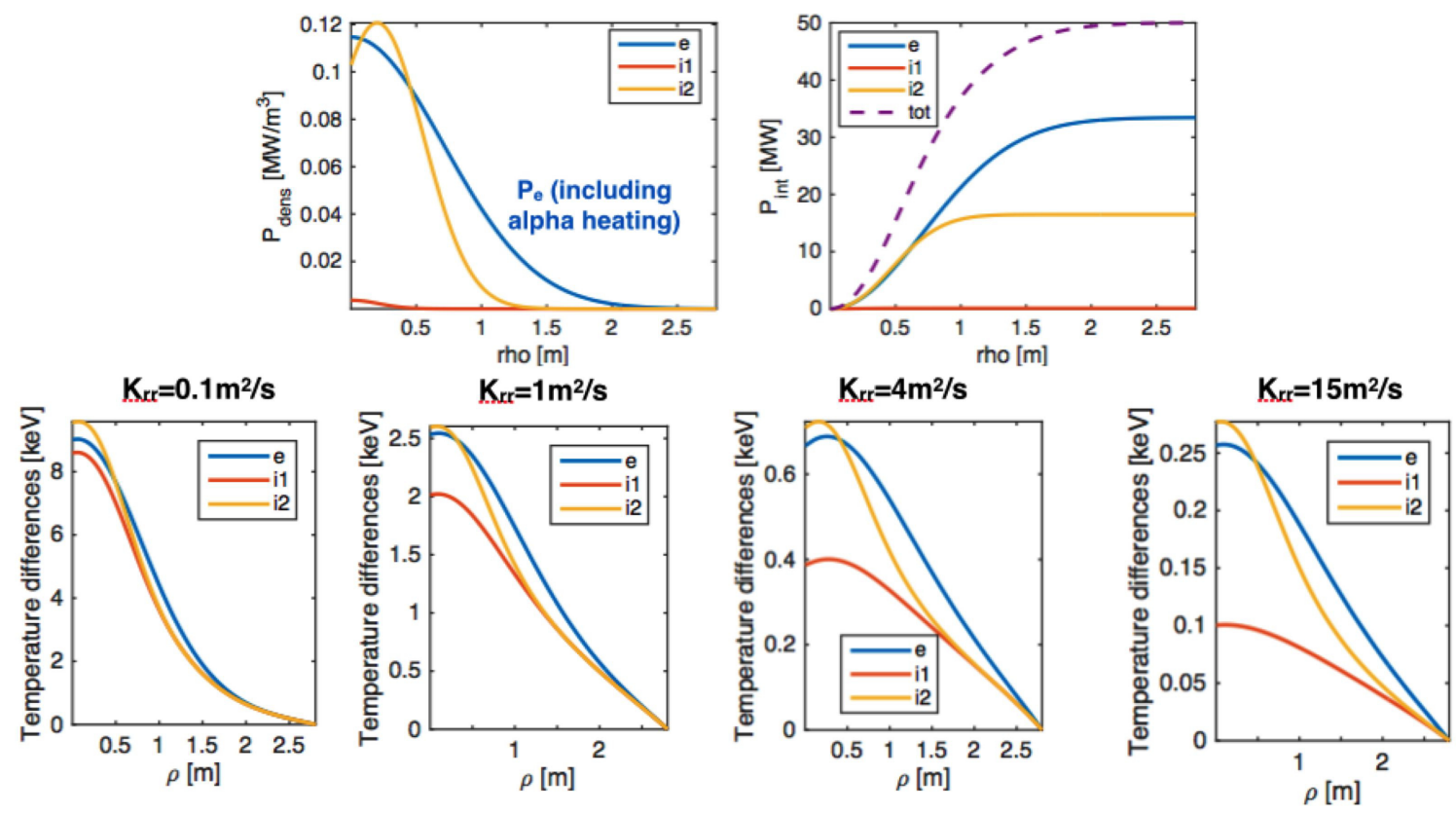

Figure 21: Local and integrated power deposition profile (top) and profiles of the temperature profile modifications (bottom) for 4 different values of the heat diffusion coefficient: $0.1,1,4$ and $15 \mathrm{~m}^{2} / \mathrm{s}$.
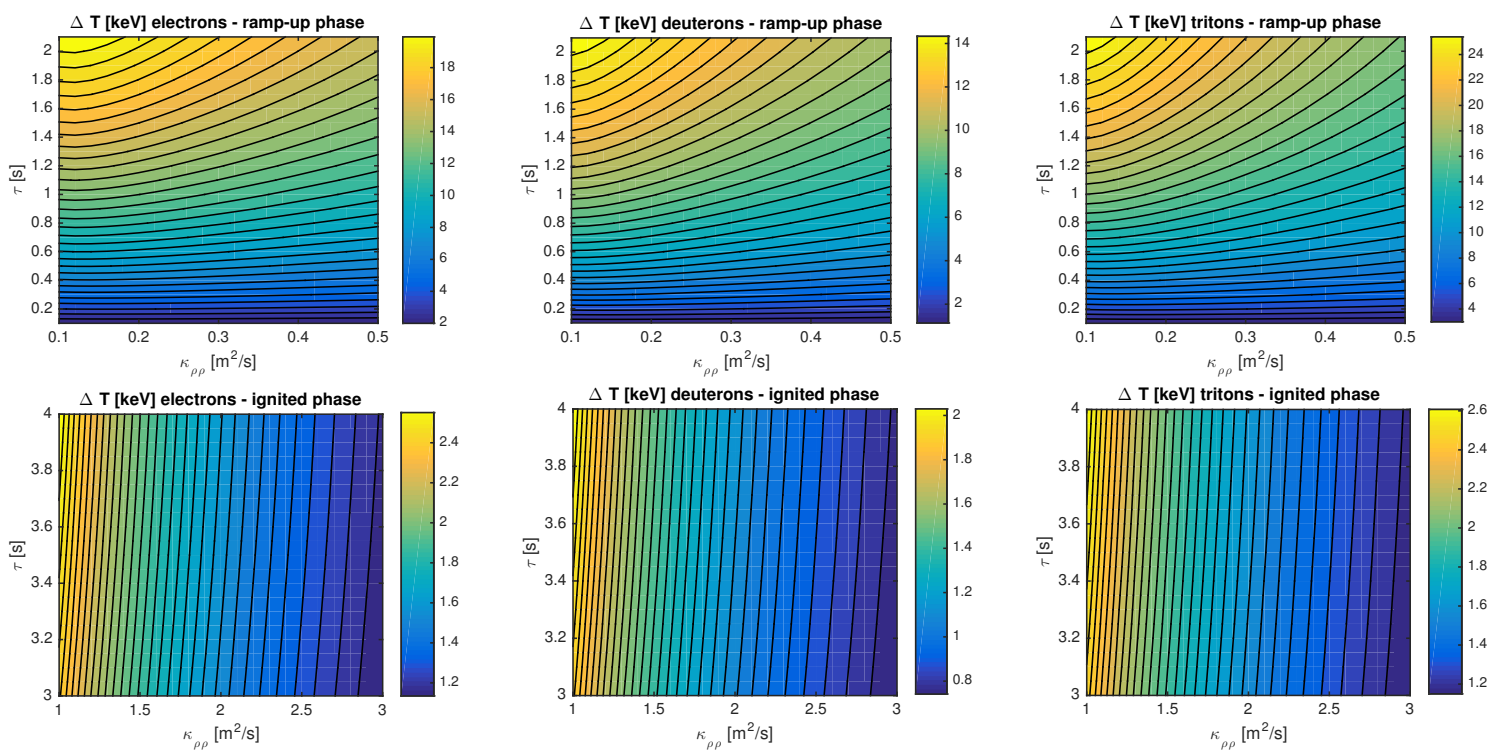

Figure 22: Core temperature modifications as a function of the heat diffusion coefficient and the energy confinement time for the ramp-up phase when the core density and temperature are modest and the plasma is in L-mode (top) and at nominal density and temperature corresponding to H-mode operation (bottom).

As can be anticipated, changing the magnetic field has a much more pronounced impact: the position of the cyclotron layers depending on the quotient of $B_{o}$ and $f$, 

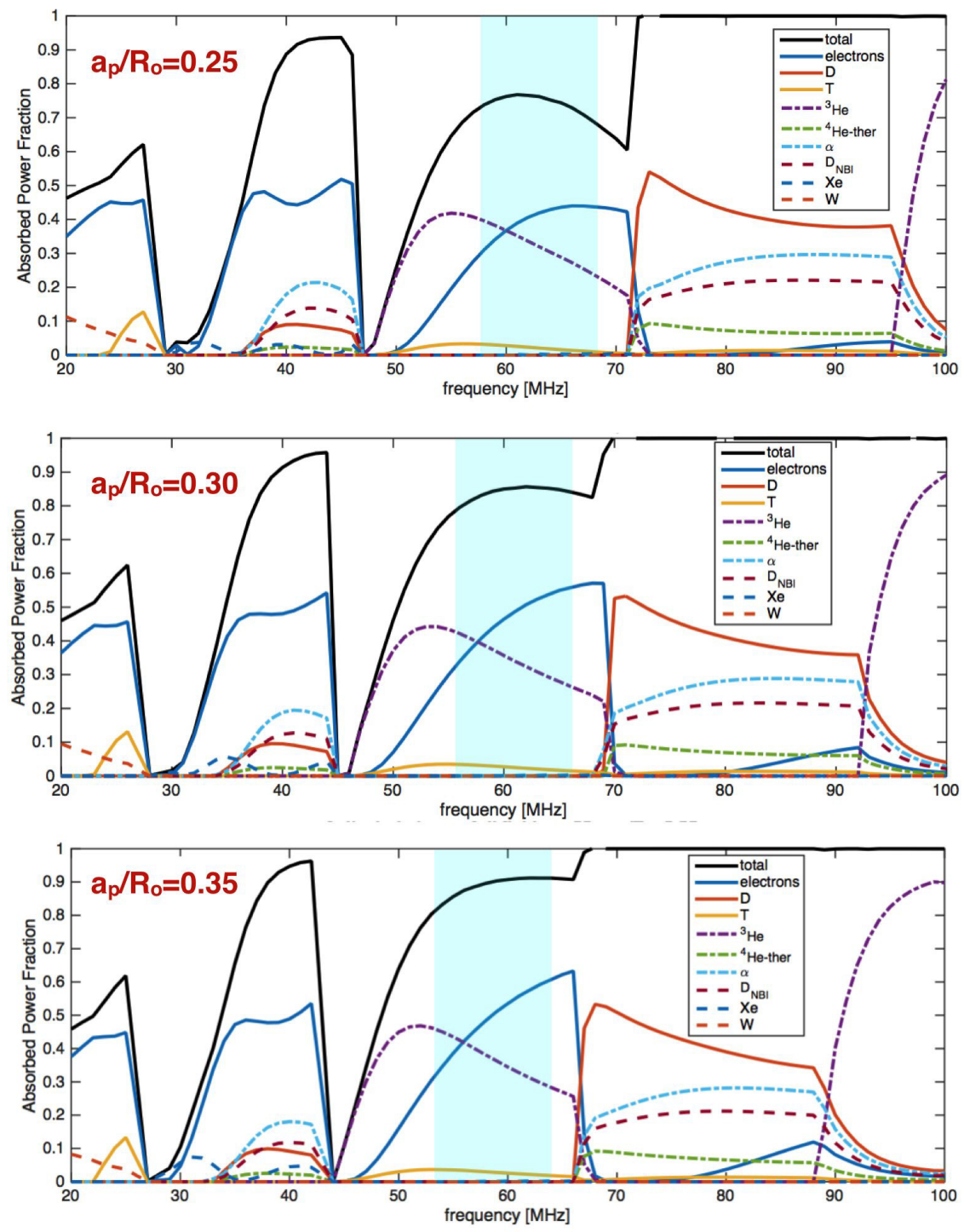

Figure 23: Single pass absorption for 3 different values of the aspect ratio: $0.25,0.3$ and 0.35 .

doubling the magnetic field requires doubling the frequency to keep the cyclotron layer at the same radial position in the machine. At low $B_{o}$, one subsequently observes a window where $N=1 \mathrm{D}$ or $\alpha$ heating is possible (up to $\approx 30 \mathrm{MHz}$ ), followed by a region where $N=2 \mathrm{~T}$ heating and $N=1{ }^{3} \mathrm{He}$ heating are the possible ion heating schemes (up to $\approx 50 M H z$ ), switching again to dominant $Z / A=1 / 2$ heating, now for $\mathrm{N}=2$ (up to $\approx 60 \mathrm{MHz}$ ). After that, a window with dominant $N=2{ }^{3} \mathrm{He}$ heating, and finally one with dominant $N=3 \mathrm{D}$-like heating. Taking the second harmonic T heating layer (highlighted in blue) as a reference, one observes the gradual shift of that interval to higher frequencies when the magnetic field increases. The antenna needs to be redesigned when $B_{o}$ is modified. This amounts to adjusting the geometry of the straps but does not pose any fundamental problem. 


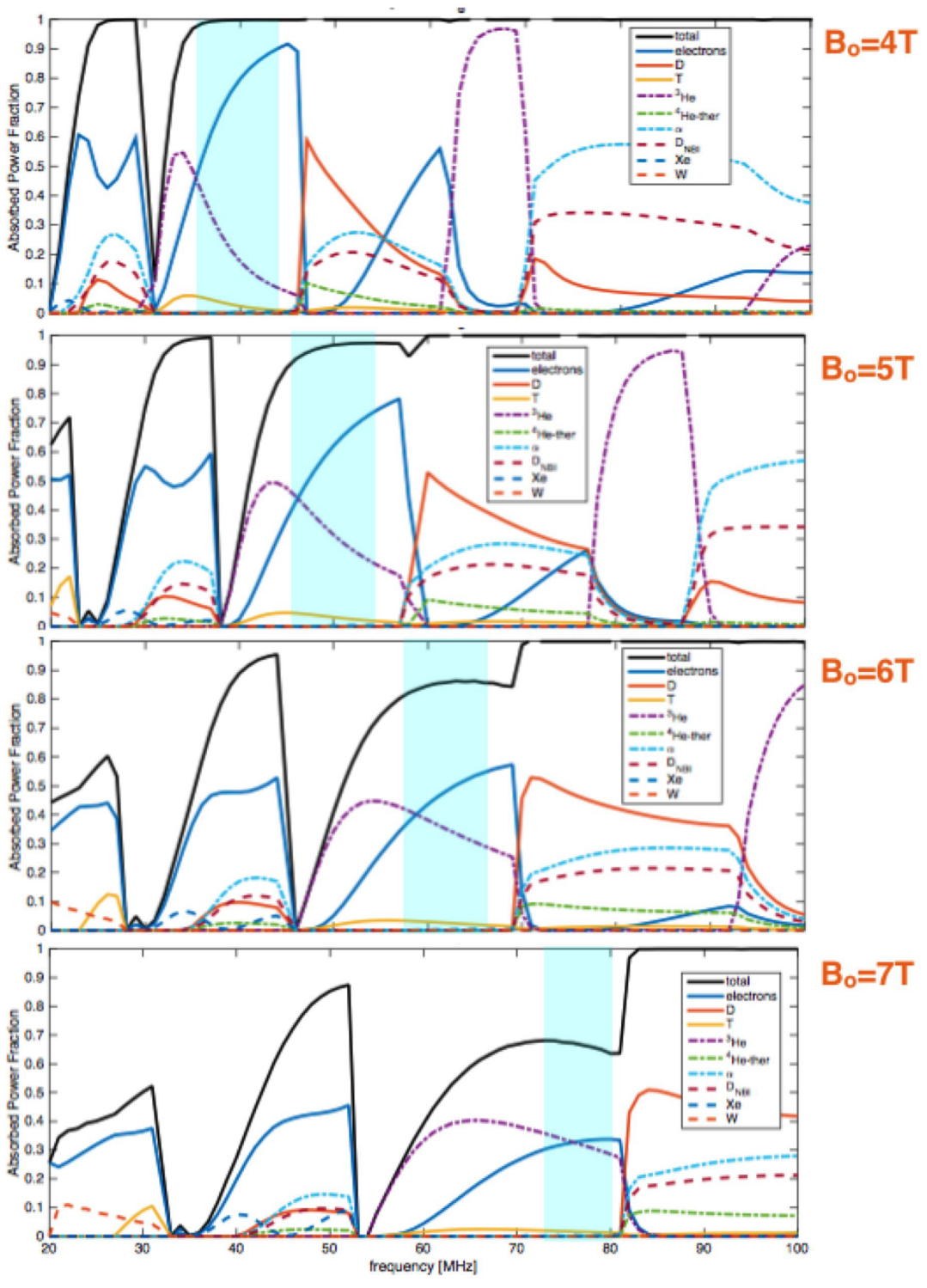

Figure 24: Single pass absorption for 4 different values of the static magnetic field: 4, 5, 6 and $7 T$.

\section{Reduced field: the early DEMO testing phase}

To limit disruption risks and to acquire knowledge of the behaviour of the plasma prior to actually igniting the machine, ITER plans early phase exploitation campaigns by gradually increasing the static magnetic field and plasma current in steps. This prudent approach is likely a path that will be taken for DEMO as well. Hence, heating schemes that can be exploited at reduced field, density and temperature need to be identified. Moreover, since activation of the machine will be avoided at this stage, ${ }^{4} \mathrm{He}$ and $H$ will be the majority gases used. In this section, some of the available RF heating schemes that can be exploited will be briefly sketched. 
As the scaling in the introductory notes indicates, harmonic heating becomes very efficient at reduced magnetic field: $P_{a b s} \propto \rho_{L}^{2(N-1)}$ where $\rho_{L}$ is the Larmor radius and $N$ the cyclotron harmonic. For the specific case $N=2$, the absorption thus scales as $1 / B_{o}^{2}$, making majority heating an order of magnitude stronger when running the machine at $1 / 3$ of the nominal magnetic field strength, $B_{o}=1.95 T$. Low field $N=2$ heating was experimentally tested in JET [90] allowing to reach $\mathrm{H}$ mode solely using ICRH power. Figure 25 illustrates this for a hydrogen majority heated at its second harmonic layer using $f=60 \mathrm{MHz}$. Total single pass absorption is achieved. Ion heating is efficient at low temperature, density as well as toroidal mode number. As Cerenkov absorption $(N=0)$ equally performs well at low $B_{o}$ (the combined Landau and TTMP absorption absorption scales as $1 / B_{o}$ ), it competes with the ions heating and becomes the dominant scheme when the plasma get hotter and denser. Also a higher parallel wave number allows the electrons to absorb wave energy more efficiently.
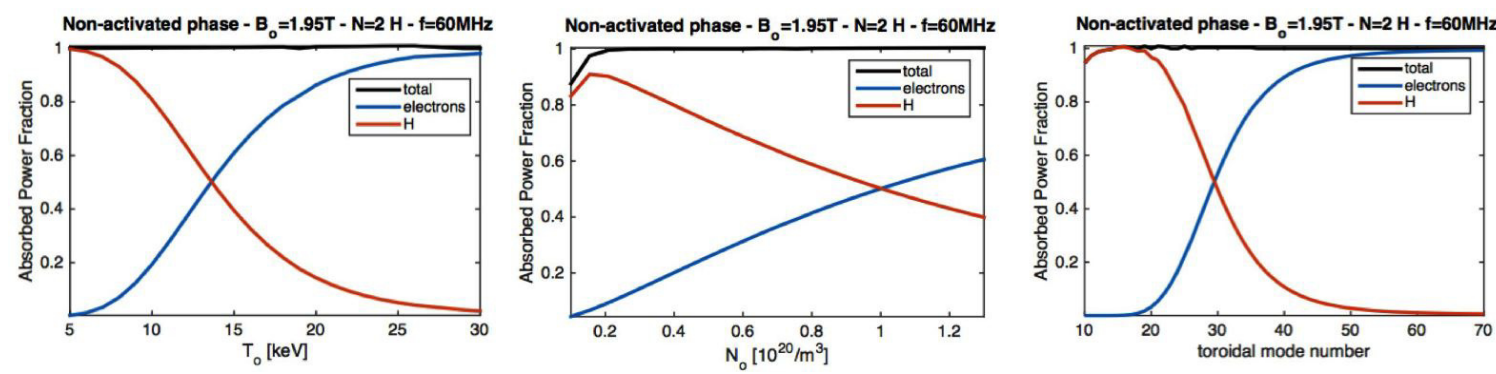

Figure 25: Single pass absorption in a $H$ majority plasma as a function of the core temperature (left), core density (middle) and toroidal mode number (right) when the magnetic field is reduced to $1 / 3$ its nominal value $\left(B_{o}=1.95 T\right) ; f=60 \mathrm{MHz}$.

A possible option for tests at half the nominal field is third harmonic ${ }^{4} \mathrm{He}$ majority heating; see Fig. 26. This is a suitable heating method at low as well as high toroidal mode number and temperature but requires a density that is not too low: at $N_{o}=$ $10^{19} / \mathrm{m}^{3}$ the single pass absorption is only $40 \%$ of the power while beyond $N_{o}=$ $5 \times 10^{19} / \mathrm{m}^{3}$ complete single pass absorption is achieved. This heating method requires to go slightly beyond the earlier proposed frequency range $(55<f[\mathrm{MHz}]<65)$ or to heat slightly off-axis when staying inside that interval.

Preparing full field operation but avoiding activation, several heating schemes are available: $H$ or ${ }^{4} \mathrm{He}$ majorities can be used, and minorities of $H$ as well as ${ }^{3} \mathrm{He}$ or ${ }^{4} \mathrm{He}$. The various candidate scenarios have been tested in current-day machines (see e.g. [91]). Testing the ${ }^{3} \mathrm{He}$ minority heating scheme can for example be done in a ${ }^{4} \mathrm{He}$ majority plasma. As ${ }^{4} \mathrm{He}$ has the same charge-over-mass ratio as $D$ and the $N=1$ ${ }^{3} \mathrm{He}$ cyclotron resonance layer coincides with the $N=2 T$ layer, this scenario can be considered to be a suitable mock-up of the $\left({ }^{3} \mathrm{He}\right)-D-T$ scheme from the RF point of view. This scheme relies on ohmic heating to lift the plasma out of the lowest temperature region where the wave heating is inefficient but becomes a suitable heating scheme (single pass absorption exceeding 50\%) when the temperature is above $15 \mathrm{keV}$. Also increased density is beneficial.

${ }^{3} \mathrm{He}$ minority heating in a $H$ plasma is an inverted heating scheme $\left([Z / A]_{\text {minority }}<\right.$ $\left.[Z / A]_{\text {majority }}\right)$ and hence the minority cyclotron layer lies behind the ion-ion hybrid 

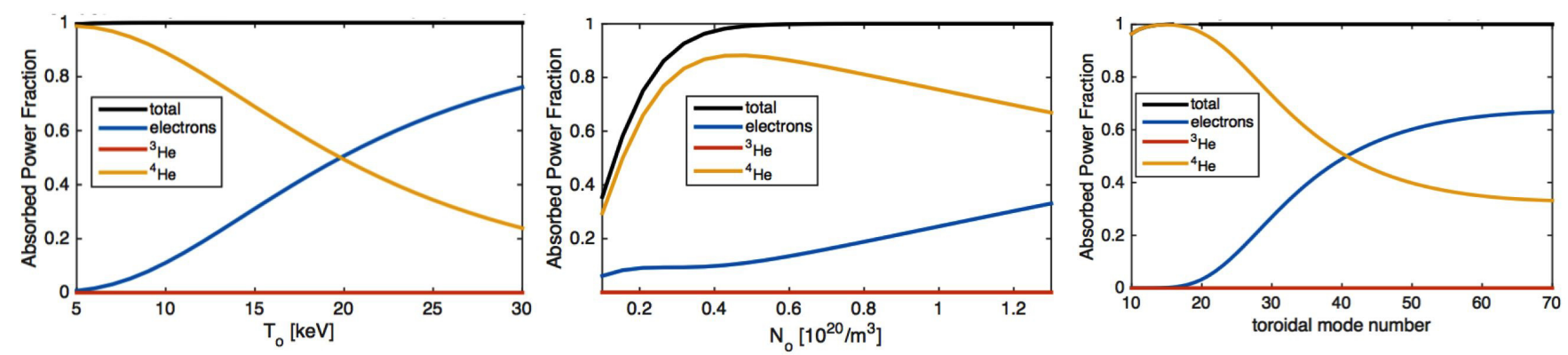

Figure 26: Single pass absorption in a ${ }^{4} \mathrm{He}$ majority plasma as a function of the core temperature (left), core density (middle) and toroidal mode number (right) when the magnetic field is reduced to $1 / 2$ its nominal value $\left(B_{o}=2.935 T\right) ; f=68 \mathrm{MHz}$.

confluence layer. As a consequence this scenario can only be used at sufficiently small

${ }^{3} \mathrm{He}$ concentration, allowing the wave power to transmit through the confluence.

\section{Discussion and conclusions}

A study has been made of the potential of ion cyclotron resonance heating for the DEMO machine. As the adopted models are simplified, a few assessments are made at the start: (i) An orbit computation learns that - although particles are not glued to magnetic surfaces - the size of DEMO permits to neglect drift orbit effects, even for high energy particles. (ii) As the wave equation solvers used assume the various species have Maxwellian distributions, a modest study based on 1-dimensional Fokker-Planck modelling was made to see which temperature is suitable for describing beams and alpha particles. $2 \mathrm{D}$ wave equation solving shows that the wave fronts typically propagate close to the equatorial plane for good absorption scenarios, allowing to mainly rely on a 1D wave equation solver. Nonetheless, it speaks for itself that a more detailed assessment would be very worthwhile to confirm the results obtained. The present study focuses on the actual ICRH dynamics while only skimming over the final fate of the power passed on from the waves to the particles: an actual transport study is left to later work.

Apart from a few exceptions, full absorption of the wave power in a single transit over the plasma is found in the high density, high temperature phase of the DEMO discharge. Only at low frequency, the absorption is not complete. Gradually increasing in frequency and using the DEMO parameters described in [4] for the ignited $D-$ $T$ burning phase, one moves from fundamental $(N=1) T$ heating $(<28 M H z)$ to fundamental $(N=1) D$ heating $(28-45 M H z)$, onwards to second harmonic $(N=2)$ $T(45-68 \mathrm{MHz})$ and then second harmonic $(N=2) \mathrm{D}$ heating $(70-90 \mathrm{MHz})$. After that a frequency window where 3rd harmonic heating is the key ion absorption scheme appears.

Two designs are currently being examined for coupling ICRH power into DEMO plasmas: A "classical" in-port option that maximally exploits the return of experience from the ITER design as well as ICRH experience on various machines, and a "traveling wave" antenna (TWA). Both antennas aim at reducing the wave induced impurity flux 
due to high electric field amplitudes in the edge but are based on different philosophies: the former avoids cross-talk between straps while the latter depends on it. Because optimal cross-talk between straps requires a sufficient distance between the antenna and the main plasma, the TWA is thought to be a natural candidate for being used in large machines. Fixing a specific design and hence an optimal operation frequency, the TWA operates well inside a bandwidth of $\approx 10 \mathrm{MHz}$ around that frequency. Hence one needs to pin down a frequency range within the available ranges. The frequency windows where $D$ absorption is possible are unavoidably spoiled by parasitic alpha particle heating by the fusion born fast ${ }^{4} \mathrm{He}$, sharing $D$ 's $Z / A$. This is the case both in the $N=1$ as in the $N=2$ case examined. For that reason the $N=2 T$ heating window is preferred; the same choice was made for ITER. As $\Omega_{3} H e=2 \Omega_{T}$, adding a ${ }^{3} \mathrm{He}$ minority to the mix adds efficient minority heating with dominant Coulomb collisional transfer to the bulk ions in that window. Not the whole frequency window is suitable, though: The lower frequency end allows ion heating but places it very far on the low field side. Too high frequency brings it too far to the high field side. The window $[55-65 \mathrm{MHz}]$ is a good compromise; core ion heating occurs at $60 \mathrm{MHz}$. In view of the large size of the machine and the fact that electron Cerenkov (Landau and transit time magnetic pumping) damping is not bound to a cyclotron layer and hence is less localised, electrons absorb a significant amount of the incoming wave power before the ion cyclotron layers are reached. Inside the chosen frequency window current drive is possible by shifting the cyclotron layer to the high field side.

A parameter study reveals that good absorption is guaranteed in a very wide range of physically relevant parameters. Whereas $N=2 T$ heating is preferred in most of the ignited phase, fundamental ${ }^{3} \mathrm{He}$ minority heating is an excellent heating scheme when the temperature is modest, either in the ramp-up phase or in the phase during which the discharge is "landed" (powered down from its ignited phase deep in H mode).

Prior to reaching the phase where it will be operated at full field and current, DEMO will likely be tested at reduced field. ICRH options are available to ensure that.

Adjusting the relative concentrations of 2 majority ions to align the region where the $E_{+}$component of the electric field - the component dominantly contributing to ion heating for not too energetic ions - peaks with the cyclotron layer of a third ion, the 3 -ion scheme offers extra perspectives. Various 3-ion candidate minorities are available that allow operating close to balanced $D-T$ mixtures; $B e,{ }^{7} \mathrm{Li}$ and $\mathrm{Ar}$ have been modelled. In spite of its potential, this scheme is only held as a back-option for 2 reasons: (i) Relying on $N=1$ heating, it requires frequencies lying in between $\Omega_{T}$ and $\Omega_{D}(f \approx 40 \mathrm{MHz})$ outside the preferred frequency range $(55-65 \mathrm{MHz})$. (ii) Whereas very efficient heating is guaranteed, alpha particle heating is unavoidable.

While ICRH ensures its primary mission - heating - during the ramp-up and landing, it fulfils other tasks (e.g. sawtooth pacing, impurity chasing and burn control) when the plasma is ignited and kept at fusion-relevant temperatures by the Coulomb collisional slowing down of fusion-born alpha particles. In between shots, ICRH is available for ensuring wall-conditioning and to start up new discharges.

To maximise the neutron yield, magnetic confinement fusion machines are operated at high densities. Current drive is an issue at such densities so alternative current drive ideas need to be explored. Fisch proposed to recharge the transformer of the tokamak by interspersing high density operation with intervals during which the density 
is brought down. Since current drive efficiencies scale inversely with density, decreasing the density by a significant factor potentially makes the efficiency high enough to envisage pulsed operation but without actually needing to interrupt the discharge.

DEMO parameters tend to change to allow incorporating new findings making the operation of the machine more efficiently. To assess the robustness of the radio frequency heating option, 2 basic parameters (the magnetic field and the aspect ratio) where scanned over a fairly wide range. This holds no fundamental problems for the $\mathrm{RF}$ system except that the antenna design needs to be adapted slightly to be optimal for the chosen parameters.

\section{Acknowledgements}

This work has been carried out within the framework of the EUROfusion Consortium and has received funding from the Euratom research and training programme 2014-2018 and 2019-2020 under grant agreement No 633053. The views and opinions expressed herein do not necessarily reflect those of the European Commission.

\section{Appendices}

\section{A Adopted wave equation solvers}

Most of the wave equation computations done for the present paper were done using the TOMCAT code [92]. Aside from being much faster than 2D wave solvers, the key advantage of using TOMCAT is that it solves the wave equation as an eigenvector problem and allows determining not only the absorption profiles but also all connection coefficients, in particular the wave transmission to the high field side and the reflection to the low field side, allowing to get an intuitive picture of the "single transit" heating efficiency of a scenario. The edge region is a difficult region to model since many assumptions commonly made for describing the wave-particle interaction break down while deeply evanescent or short wavelength modes that are numerically difficult to trace can be excited. Non-linear effects are likely to play a key role but the exact nature of the dominant mechanisms is presently unclear. Finally, wave induced plasma deformations as well as the presence of metallic objects impacting on the dynamics need to be accounted for. Close to the launcher - and in contrast to what is the case in the main plasma - electric fields are significant while the temperature is low, resulting in RF effects being on par with the magnetic confinement forced by the static magnetic field. Over the last years, authors have started to get a grip on the near-antenna-field behaviour but the fate of the power lost in the edge is currently still poorly understood. Focussing on the core region only, TOMCAT provides the amount of power leaving the domain of interest after a full transit of the waves through it, hereby indirectly giving an idea of how many transits the wave needs over the plasma to fully lose the energy it carries.

2D wave solvers - in contrast - allow to study the impact of wave diffraction and focussing but are solved accounting for the metallic vessel wall which acts as a Faraday cage, forcing all power launched by the antenna to be lost in the plasma so that only the relative power various particle populations absorb can be assessed, edge absorption 
mechanisms not being accounted for. 2D wave solvers (dominantly CYRANO [93, 94] and an all-FLR solver still under development) will be used to complement the picture TOMCAT provides with aspects that are intrinsically 2-dimensional. These codes account for the actual magnetic geometry, and for the fact that the plasma has a plasma current and hence a poloidal magnetic field. In view of the size of the machine and the fact that the antenna location is in or near the equatorial plane, the wave pattern is essentially 1-dimensional and the 2 approaches yield similar results; 'similar' as opposed to 'directly comparable' since TOMCAT does not account for the antenna nor for the metallic walls surrounding the plasma.

\section{B Adopted Fokker-Planck solvers}

Two Fokker-Planck solvers will be used. StixReDist [34] solves a set of coupled 1dimensional steady state Fokker-Planck equations for the various plasma constituents' distribution functions, assumed to be isotropical. The general nonlinear collision operator [95] is adopted and hence both minorities and majorities can be treated. StixReDist adopts the philosophy of Stix [11] to solve the Fokker-Planck equation. This technique allows to semi-analytically solve the second order Fokker-Planck equation as a first order one upon assuming there are no particle sources nor losses so that the flux - of the form $A F_{o}+B d F_{o} / d v$ - is conserved. As the flux at infinity needs to be zero, the solution can be determined up to a constant factor: $F_{o} \propto \exp \left[-\int d v B / A\right]$. The constant is determined integrating the obtained result over velocity space and equating it to the density. Whereas Stix's solution only holds for minorities and for fundamental cyclotron heating, StixReDist retains the full Kennel-Engelman operator and hence allows to study wave heating at any cyclotron harmonic.

StixReDist cannot be used to compute the distribution of beam populations or for fusion-born alpha particles. Such distributions are found solving the Fokker-Planck equation including a particle source, and a particle and energy loss term relying on the finite element approach [96].

\section{Adopted transport solver}

The present paper limits itself to studying the direct impact ICRH waves have on a tokamak plasma i.e. on providing the heat sources entering the transport equations rather than on making a detailed study of the effect of energy transport itself. Nonetheless, a crude assessment of whether the coupled MWs of power have an impact is useful. Rather than solving the actual transport equations a simplified model will be adopted to compute the temperature profiles consistent with a given power deposition profile. It assumes a constant diffusion and convection throughout the discharge, and - as the various species are Coulomb collisionally transferring energy to each other when their temperatures differ - contains an the equipartition term. A single source term $P_{j}$ (the profile provided by the Fokker-Planck computation) is included per type of species $j$. Especially at high density, this term is a key ingredient of the dynamics, allowing to transfer power between species and attempting to annihilate the temperature difference. A simple loss term - characterized by a constant loss time $\tau_{j}$ - was adopted and it was assumed a steady state regime has been reached $\left(\partial T_{j} / \partial t=0\right)$. For each of the 
species $j$ the plasma is constituted of, the energy transport equation reads

$$
\begin{gathered}
\frac{d}{d \rho}\left[\left[<J|\nabla \rho|^{2}>K_{\rho \rho} N_{j} \frac{d}{d \rho}\right]+\left[<J|\nabla \rho|>V_{\rho} N_{j}+<J|\nabla \rho|^{2}>K_{\rho \rho} \frac{d N_{j}}{d \rho} \frac{d}{d \rho}\right]\right] T_{j} \\
+<J>\left[N_{j} \sum_{j^{\prime}}\left[\nu_{c}^{j^{\prime} / j}\left(T_{j}^{\prime}-T_{j}\right)\right]+P_{j}-\frac{N_{j} T_{j}}{\tau_{j}}\right]=0
\end{gathered}
$$

in which $J$ is the Jacobian from cartesian to toroidal coordinates, $<\ldots>$ represents the poloidal average over a magnetic surface, $\rho$ is the magnetic surface labeling parameter, $K_{\rho \rho}$ is the heat diffusion coefficient, $V_{\rho}$ is the heat convection (pinch velocity) coefficient, $N_{j}$ is the density and $\nu_{c}^{j^{\prime} / j}$ is the frequency of the Coulomb collisions between species $j$ and species $j^{\prime}[97]$.

The set of transport equations is solved imposing $d T_{j} / d \rho$ at $\rho=0$ and a negligibly small temperature at the outer edge (and hence allowing energy to leak out through the edge).

\section{Orbits}

In the present paper tools are adopted that assume that the particles do not deviate from magnetic surfaces while in reality they have a drift away from them because the magnetic moment $\mu=m v_{\perp}^{2} /\left[2 B_{o}\right]$ (where $v_{\perp}$ is the magnitude of the velocity perpendicular to the confining magnetic field) is an adiabatic invariant (see e.g. [98]). Hence a minimal check that needs to be performed to show the adopted tools are adequate is to assess the width of relevant fast particles - be it fusion born alpha particles, RF induced tail particles or beam particles. The more energetic the particle and the higher its perpendicular energy, the more its drift away from magnetic surfaces is pronounced. Zero-banana-width assumptions by definition assume particles are glued to magnetic surfaces on the radio frequency as well as on the bounce time scale; they may be lost on the transport time scale. Hence one should check if first orbit losses are to be expected for the fast particles that are expected to be present. As fast particles will dominantly be created in the core of the plasma up to $1 / 3$ of the minor radius, the high energy orbits drawn are assumed to be created this region. A consequence of the zero-orbit-width assumption is that the power density is predicted to be more localised than it is in reality. In particular, the RF power density close to the magnetic axis is somewhat overestimated. Since the energy of high energy tails is in direct relation to the $\mathrm{RF}$ power density rather than to $\mathrm{RF}$ power, it makes sense to assess the orbit width and smoothen the zero-orbit-width power deposition profile obtained over a typical orbit width before using it as an input to the Fokker-Planck solver when assessing the fate of the wave power.

Figure 27 depicts 3 orbits of the thermal (left subfigures) as well as heated (right subfigures) tritons. Orbits (or parts of the orbits for trapped particles) with parallel velocities that are bigger than zero are drawn in red while counter-moving orbits are in blue. The toroidal angular moment coincides with the poloidal flux when the parallel velocity vanishes. The corresponding magnetic surfaces are indicated by a dash-dotted black line. For passing particles the sign of the parallel velocity does not change along 
the orbit and hence the full orbit lies inside or outside the reference magnetic surface depending on its sign. The orbit width of thermal particles at full field (top left subfigure) is very small compared to the minor radius. At $1 / 3$ field or when heated (the orbit of a $200 \mathrm{keV}$ triton is depicted on the top right), the orbit is about $10 \%$ of the minor radius. At reduced field, tail particles have a width of about $20 \%$ of the minor radius.

In view of their much higher energies, the orbits of fusion born $\alpha$ particles deviate further from magnetic surfaces. Even if they would be heated to energies of $15 \mathrm{MeV}$ (their orbits now having a width of $1 / 4$ th of the minor radius), fusion $\alpha$ 's born in the DEMO core are still well confined, however (plots not shown). This beneficial characteristic is due to the fact that DEMO is a large machine that can be operated at high current.

One can conclude that first orbit losses are not be expected to be an issue so that the orbit effects can - to leading order - be neglected in this study except for a smoothing of deposition profiles obtained from zero-orbit-width codes over the expected orbit width. For $50 M W$ of RF power and anticipating that the ion deposition profile is close to the cold cyclotron layer placed at or very near to the centre of the plasma, RF power densities up to about $0.6-0.7 \mathrm{MW} / \mathrm{m}^{3}$ are to be expected.

\section{E The alpha particle distribution function}

Fig.28 gives an idea of what the alpha particle distribution looks like for fundamental and second harmonic heating, implicitly assuming the proper frequency choice has been made to affect alphas by RF waves. Adopting the confinement time suggested in the DEMO reference as energy and particle confinement time $\left(\tau_{P}=\tau_{E}=3.64 \mathrm{~s}\right)$, about $60 \%$ of the particles are in the thermal population while this population only carries $4 \%$ of the energy when the $\alpha$ 's are RF heated at their second harmonic cyclotron frequency with $P_{R F}=0.2 \mathrm{MW} / \mathrm{m}^{3}$. Figure 28 depicts $v^{2} F_{o}$ and $v^{4} F_{o}$ (the local density and the local energy density up to the factor $m / 2$ ) in velocity space as a function of the energy. To get an idea of the fast and slow subpopulations, a Maxwellian is fitted to the distribution in the low energy region. The plots show the total contributions as well as the thermal and fast subpopulations. The distribution itself is dominantly nonzero only in the lower energy region (not shown). Plotting the local density or energy shows the importance of the high energy subpopulation. In view of the finite RF power applied, a tail extends well beyond the birth energy of $3.45 \mathrm{MeV}$. The dominant contribution for the energy density comes from the source region but a high energy tail is observed up to $7 \mathrm{MeV}$. The fast subpopulation has an effective temperature of $1.2 \mathrm{MeV}$ while the fit to the thermal subpopulation yields and effective temperature matching that of the background. Because of the competition between RF and Coulomb collisional diffusion, the distribution is not a Maxwellian, not even in the thermal region. For $N=2$ the deformation of thermal population is weaker than for $N=1$. The alpha particle energy distribution is similar for the 2 heating schemes. Subtle differences are in line with expectations: more energetic tails are formed when adopting harmonic heating (top right subplot) while deviations from a Maxwellian and a shift towards higher energies is observed at modest energies (bottom right subplot). 

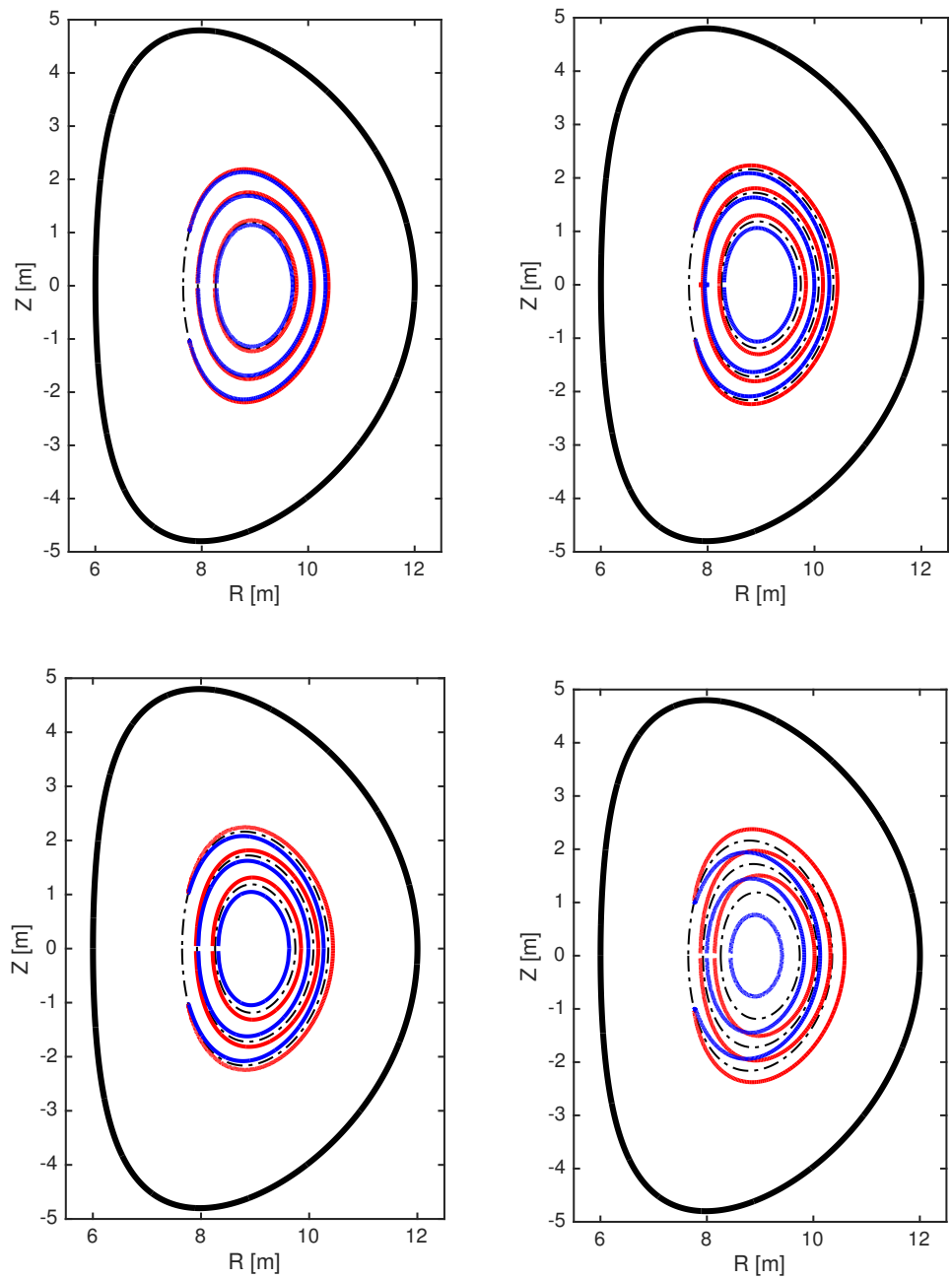

Figure 27: Orbits of tritons in DEMO. The top plots are for full field $\left(B_{o}=5.855 T\right)$ and current $\left(I_{p}=17.75 \mathrm{MA}\right)$, the bottom ones for $1 / 3$ field and current. The left figures are for $E_{T}=20 \mathrm{keV}$ and the right subfigures for $E_{T}=200 \mathrm{keV}$; red lines are for $v_{/ /}>0$ while blue lines are for $v_{/ /}<0$. 

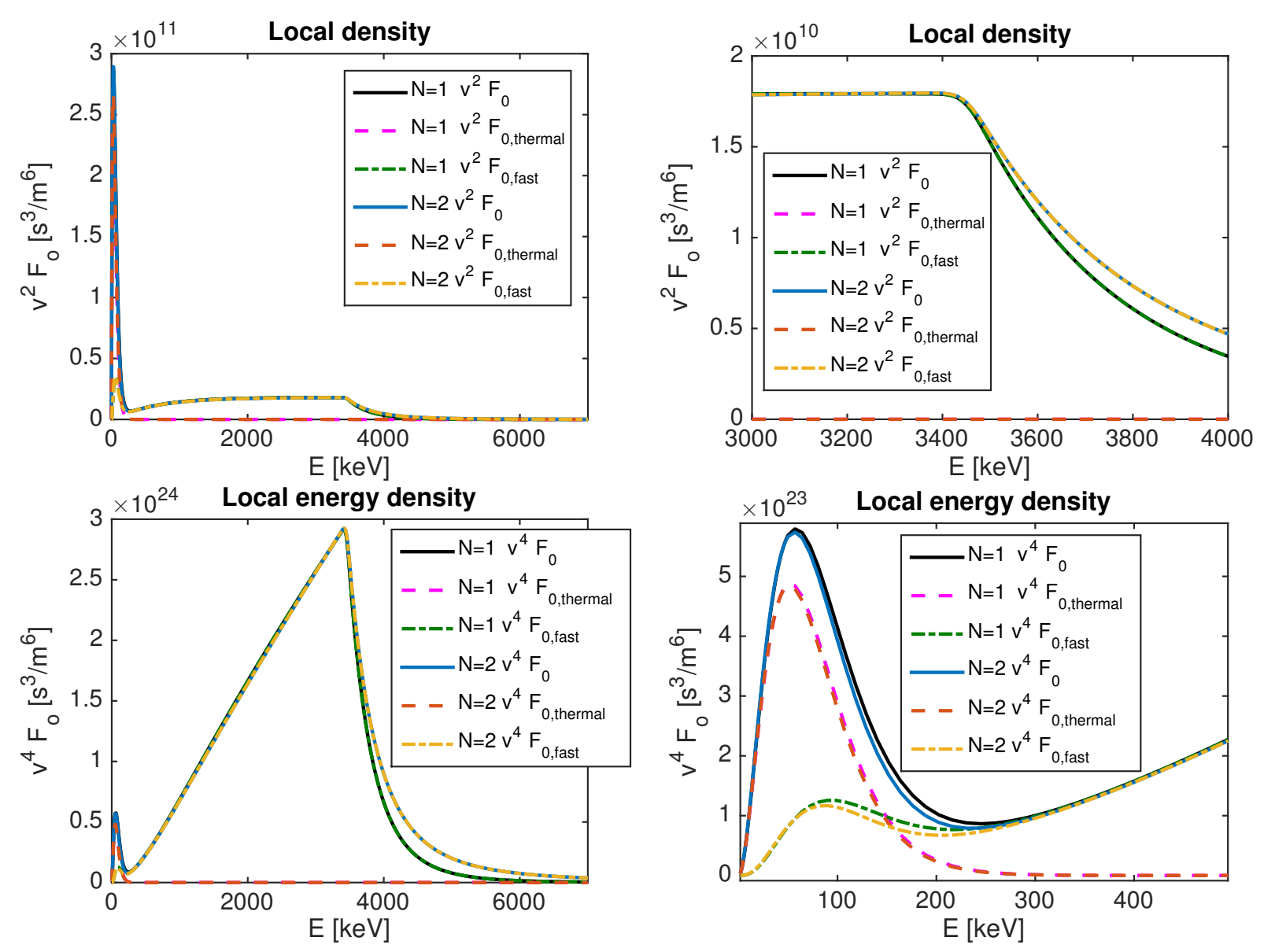

Figure 28: Alpha particle $v^{2} F_{O}$ (local density divided by $4 \pi$ ) and local energy density $v^{4} F_{o}$ (local energy density divided by $4 \pi m_{\alpha}$ where $m_{\alpha}$ is the alpha particle mass) for $P_{R F}=0.2 \mathrm{MW} / \mathrm{m}^{3}$ and $X\left[{ }^{4} \mathrm{He}\right]=4 \% ; \tau_{P}=\tau_{E}=3.64 \mathrm{~s}$. The right subplots are zooms of relevant parts of the left subplots.

\section{F Equivalent Maxwellian temperature of the al- pha and beam particle populations}

Alpha particles are born from the $D-T$ fusion reactions. Their birth energy is $3.45 \mathrm{MeV}$. They slow down - mainly on electrons - so their distribution contains a thermalised subpopulation, a slowing down subpopulation and high energy part. When RF heated, a very high energy tail is formed, as is briefly discussed in Appendix E.

The alpha particle distribution deviates significantly from a Maxwellian while the wave equation solvers adopted for the present work assume all populations are Maxwellian. A suitable temperature therefore has to be identified to account for the presence of the alpha's. Koch proposed to adopt the temperature for which the RF power density of the substituting Maxwellian matches that of the distribution [99]. He modeled the alpha particles by the slowing down distribution introduced by Stix [11], exploiting a simplified collision operator that does not conserve particles at $v=0$ and acts as a particle sink, removing the thermal subpopulation. Solving the actual Fokker-Planck equation allows to get a more suitable guess, including the impact of the actual loss 
term, the finite RF power and the full collision operator. For 0, 0.2, 0.4 and $0.6 M W$ one readily finds that the temperatures allowing to match the RF power density are $376,444,518,601$ and $673 \mathrm{keV}$, respectively, for heating at the fundamental cyclotron frequency $(N=1)$ and $434,552,682$ and $780 \mathrm{keV}$ for $N=2$. Different loss times allow more or less time for the thermal subpopulation to form. When varying the confinement time for $1 s$ to $6 s$ in steps of $1 s$, the required temperatures to match the RF power density of $0.2 \mathrm{MW} / \mathrm{m}^{3}$ are $915,628,500,419,368$ and 333, respectively, for $N=1$ and $916,701,598,532,502$ and $472 \mathrm{keV}$ for $N=2$. A Maxwellian equivalent temperature of the $\alpha$ 's of $600 \mathrm{keV}$ will uniformly be adopted in the RF absorption efficiency study. Similarly, the $1 \mathrm{MeV} D$ beams will be modeled as $200 \mathrm{keV}$ Maxwellians. The adopted equivalent temperature is significantly different from the effective temperature $(2 / 3$ of the average energy) of the $\alpha$ population, underlining that matching the shape of the energy density is not sufficient to matching the RF absorption efficiency. The effective temperatures are in the range of $400 \mathrm{keV}$. Remind that fundamental cyclotron heating dominantly affects the thermal region while harmonic heating mostly influences particles that are already energetic. Hence, a Maxwellian with a temperature identified with the overall effective temperature of a non-Maxwellian population is only a crude proxy of the actual distribution.

\section{References}

[1] W. Biel et al., Fusion Engineering 85 Design" 123 (2017) 206

[2] T. Donné and W. Morris, European Research Roadmap to the Realisation of Fusion Energy, https://www.euro-fusion.org/fileadmin/user_upload/EUROfusion/ Documents/2018_Research_roadmap_long_version_01.pdf

[3] G. Federici et al., Fus. Eng. Des. 109-111 (2016) 1464-1474

[4] J. Morris, Physics and Magnets baseline February 2018 (2018) https://idm.eurofusion.org/?uid=2MGYZP\&version=v1.0

[5] M. Kovari, et al., Fusion Eng. Des. 89 (12) (2014) 3054-3069.

[6] https://www.exponentialinvestor.com/commodities/helium-3-prices-are-out-ofthis-world/

[7] D. Van Eester et al., Nucl. Fusion 42 (2002) 310-328

[8] H. Zohm et al., Assessment of HECD System Capabilities for DEMO, 40th EPS Conference on Controlled Fusion and Plasma Physics, 2013

[9] M. Porkolab, Proc. 6th Symposium on Advances in Plasma Physics, May 1992

[10] J. Hosea et al., EPJ Web of Conferences (2017) 15702003

[11] T.H. Stix, Waves in Plasmas, Springer-Verlag, New York, 1992

[12] F.W. Perkins, Nuclear Fusion (17) (1977) 1197

[13] Ye. O. Kazakov et al, Nucl. Fusion 55 (2015) 032001

[14] Ye. O. Kazakov et al, Efficient generation of energetic ions in multi-ion plasmas by radio-frequency heating, Nat. Phys. (https://doi.org/10.1038/NPHYS4167)

[15] D. Anderson et al., Fusion Technology, 23:1 (1993) 5-41, DOI: 10.13182/ FST93A30117 
[16] F. Wising, On Fast-Ion Transport and Burn Control in Tokamaks, Technical Report 256 (PhD thesis), Chalmers University of Technology, Göteborg (1994)

[17] R. Ragona \& A. Messiaen, Nucl. Fusion 56 (2016) 076009

[18] M.Valisa et al., Proc. 49th Annual Meeting of the APS, Division of Plasma Physics (2007) Orlando (USA)

[19] F. J. Casson et al., Plasma Phys. Control. Fusion 57 (2015) 014031

[20] A. Messiaen et al., Proc. 3rd joint Varenna-Grenoble Int. Symposium, Vol. 1 (1982 ) 243

[21] V. Fuchs et al., Phys. Plasmas 2 (1995) $1637 ? 47$

[22] M.-L. Mayoral et al., Nucl. Fusion 46 (2006) S550-S563

[23] D. Van Eester et al., Plasma Phys. Control. Fusion 54 (2012) 074009

[24] A. M. Messiaen et al, Plasma Phys. Control. Fusion 35 (1993) A15

[25] A. Krasilnikov, Plasma Phys. Control. Fusion 51 (2009) 044005

[26] J. Ongena et al., EPJ Web of Conferences 157 (2017) 02006

[27] H. Zohm, Proc. 40th EPS Plasma Physics Conference, Espoo (Finland) 37D (2013) O3.108

[28] D. Van Eester and E. Lerche, LPP-ERM/KMS Report 142 (2012)

[29] Ye. O. Kazakov et al., Plasma Phys. Control. Fusion 57 (2015) 025014

[30] F. Wagner et al., DEMO ICRH Review Panel Recommendations Report (2018)

[31] N.J. Fisch, J. Plasma Phys. 76 (2010) 627-6345

[32] D.A. Ehst et al., Nucl. Fusion 31 (1991) 1933-8

[33] V.P. Bhatnagar et al, Nucl. Fusion 34 (1994) 1579

[34] D. Van Eester \& E.A. Lerche, Plasma Phys. Control. Fusion 53 (2011) 092001

[35] A. Messiaen \& R. Ragona, EPJ Web of Conferences 157 (2017) 03033

[36] V. Bobkov et al., Plasma Phys. Control. Fusion 59 (2017) 014022

[37] I. Monakhov et al., textitEPJ Web of Conferences 157 (2017) 03035

[38] A. Messiaen et al., Nucl. Fusion 50 (2010) 025026

[39] T. Ogawa et al., Nuclear Fusion 41 (2001) 1767

[40] R. Ragona et al., A Travelling Wave Array system as solution for the ICRF heating of DEMO, Preprint: 2018 IAEA Fusion Energy Conference, Gandhinagar [FIP/P8-11]

[41] R.J. Perkins et al., Plasma Phys. Control. Fusion 61 (2019) 045011

[42] A. Messiaen \& R. Ragona, Proc. 43rd EPS Conference on Plasma Physics, Leuven (2016) P2.066.

[43] V. Bobkov et al., Nucl. Fusion 56 (2016) 084001

[44] R. Ragona, Progress on the design of a DEMO high power ICRH travelling wave antenna mock-up to be tested on WEST, to be published in Proc. 23rd RF Power in Plasmas Conference, Hefei, 2019, P3.29 
[45] D. F. H. Start et al., Nucl. Fusion 39 (1999) 321,

[46] G. A. Cottrell et al., Nucl. Fusion 39 (1999) 389

[47] http://fusionwiki.ciemat.es/wiki/Scaling*law

[48] J.A. Snipes et al., Plasma Phys. Control. Fusion 42 (2000) A299

[49] Y. R. Martin et al., J. Phys.: Conf. Ser. 123 (2008) 012033

[50] C.F. Maggi et al., Plasma Phys. Control. Fusion 60 (2018) 014045

[51] R. Singh et al., Phys. Plasmas 21 (2014) 062503

[52] W. Fundamenski et al., Nucl. Fusion 52 (2012) 062003

[53] R. Koch et al., Proc. 26th EPS Conf. on CFPP, Maastricht 1999, ECA 23J (1999) 745

[54] J. R. Luo and the HT-7 Team, Proc. 18th Fusion Energy Conference, Sorrento 2000, Italy, IAEA-CN-77 EXP1/01

[55] X. Litaudon et al, Nucl. Fusion 57 (2017) 102001

[56] D. Douai et al., Journal of Nuclear Materials 415 (2011) S1021-S1028

[57] T. Wauters et al., Nucl. Fusion 53 (2013) 123001

[58] T. Wauters et al., ICRF Discharge Production for Ion Cyclotron Wall Conditioning on JET [EX/P5-21], paper presented at 25th IAEA Int. Conf. on Fusion Energy, St. Petersburg 2014

[59] A. Hakola et al., Nucl. Fusion 57 (2017) 066015

[60] N. Nazarov et al., Sov. J. Plasma Phys. 13 (1987) 871-873

[61] T. Wauters et al., Proc. 22nd Topical Conference on Radio-Frequency Power in Plasmas, EPJ Web of Conferences 157 (2017) 03064

[62] M. Tripsky, Fundamentals of Discharge Initiation by ICRF Antennas in Fusion Devices, PhD Thesis (2017), Ghent University, Ghent

[63] L.E. Zakharov et al., Phys. Plasmas 19 (2012) 055703

[64] G. Van Oost et al., Proceedings EPS Conf., Bournemouth 1995

[65] M. Goniche et al., Proc. 41th EPS conf. on Contr. Fusion 85 Plasma Phys., Berlin, Germany 45 (2014) O4.129

[66] E. Lerche et al, Nucl. Fusion 56 (2016) 036022

[67] R. Neu et al., Plasma Phys. Control. Fusion 44 (2002) 811

[68] R. Bilato et al., Nucl. Fusion 54 (2014) 072003

[69] C. Angioni et al., Nucl. Fusion 57 (2017) 022009

[70] E. Joffrin et al., Overview of the JET preparation for Deuterium-Tritium Operation, Preprint: Proc. 2018 IAEA Fusion Energy Conference, Gandhinagar [OV/13]

[71] V. Bobkov et al., Nucl. Fusion 50 (2010) 035004

[72] D.A. D'Ippolito et al. Phys. Plasmas 13 (2006) 102508

[73] D.A. D'Ippolito et al., Phys. Plasmas 15 (2008) 102501 
[74] J. R. Myra and D. A. D'Ippolito, Phys. Plasmas 22 (2015) 062507

[75] D. Van Eester, K. Crombé, and V. Kyrytsya, Plasma Phys. Control. Fusion 55 (2013) 055001

[76] B. Van Compernolle et al., Fast wave experiments in LAPD in support of fusion, Preprint: Proc. 2018 IAEA Fusion Energy Conference, Gandhinagar [EX/P6-30]

[77] D. Van Eester, K. Crombé, and V. Kyrytsya, Plasma Phys. Controlled Fusion 55 (2013) 025002

[78] D. Van Eester and K. Crombé, Phys. Plasmas 22 (2015) 122505

[79] S. von Goeler et al., Phys. Rev. Lett. 197433 (1974) 1201

[80] D. Park and D.A. Monticello, Nuclear Fusion 30 (1990) 2413

[81] V. P. Bhatnagar et al, Plasma Phys. Control. Fusion 31 (1989) 2111

[82] O. Sauter et al., Phys. Rev. Lett. 88 (2002) 105001

[83] M. Lauret et al., Nucl. Fusion 52 (2012) 062002

[84] E. Lerche et al, Nucl. Fusion 57 (2017) 036027

[85] F. Köchl et al., Plasma Phys. Control. Fusion 60 (2018) 074008

[86] P.C. de Vries et al Nucl. Fusion 58 (2018) 026019

[87] E.A. Lerche et al., ICRH scenarios for JET-ILW DT operation (DTE2), in preparation

[88] ITER Physics Expert Groups et al, ITER Physics Basis, Chapter 1, Nucl. Fusion 39 (1999) 2137

[89] ITER Physics Expert Groups et al, ITER Physics Basis, Chapter 2, Nucl. Fusion 48 (2008) 099801

[90] E. A. Lerche et al., AIP Conference Proceedings 1689 (2015) 040003

[91] E.A. Lerche et al. Plasma Phys. Control. Fusion 53 (2011) 124019

[92] D. Van Eester \& R. Koch, Plasma Phys. Control. Fusion 40 (1998) 1949-75

[93] P.U. Lamalle, Plasma Phys. Control. Fusion 39 (1997) 1409

[94] E.A. Lerche, Plasma Phys. Control. Fusion 51 (2009) 044006

[95] C.F.F. Karney, Comput. Phys. Rep. (1986) 4183-244

[96] D. Van Eester \& E.A. Lerche, in preparation

[97] D.L. Book, NRL Plasma Formulary, Naval Research Laboratory (1990) Washington

[98] R. Balescu, Transport Processes in Plasma Volumes 1 \& 2, Elsevier Science \& Technology, Oxford, 1988

[99] R. Koch, Physics Letters A 157 (1991) 399-405 